\title{
Minding the Matter of Psychokinesis: A Review of Proof- and Process-Oriented Experimental Findings Related to Mental Influence on Random Number Generators
}

\author{
Bryan J. Williams \\ Psychical Research Foundation \\ bryanwilliams@psychicalresearchfoundation.com
}

Submitted November 13, 2021; Accepted November 17, 2021; Published December 30, 2021

https://doi.org/10.31275/20212359

Creative Commons License CC-BY-NC

Abstract-Many experiments have been conducted over the past eight decades to explore whether the ostensible psychic ability of psychokinesis (PK, or "mind over matter") might be a genuine human potential, and the most extensive of these have involved attempts to mentally influence the output of electronic, binary-bit random number generators (RNGs). Research of this type can generally be divided into two lines: proof-oriented (concerned with the accumulation and statistical evaluation of data from controlled experiments designed specifically to test for the presence of PK effects on the microscopic scale) and process-oriented (concerned with conducting exploratory experiments designed to systematically vary certain test conditions in order to search for and identify any physical, biological, and psychological factors which might have a role in improving or moderating PK effects). To help orient novice investigators and crossdisciplinary researchers who may be considering work along these lines (as well as to offer some initial guiding insight on possible directions for future research), this paper provides a general review of some of the notable proof- and process-oriented findings that have been obtained to date in experimental microscopic PK research using RNGs. The review generally indicates that although a considerable amount of proof-oriented data for micro-PK has accumulated over the years, the relatively sparse amount of process-oriented data available at present leaves many open questions regarding the underlying factors involved, providing ample opportunity for novice investigators and cross-disciplinary researchers to make valuable research contributions in the future.

Keywords: anomalous perturbation, microscopic psychokinesis (micro-PK), mind-matter interaction, random number generator (RNG) 


\section{INTRODUCTION}

Could humans be capable of affecting tangible objects and other forms of matter present in the physical world simply through mental means alone? Although such a question might initially seem totally illogical (or even quite ridiculous) to some scientists, it can be argued that the matter of whether psychokinesis ( $P K$, often colloquially referred to as "mind over matter") exists as a genuine form of human ability is one which could be worth pondering even momentarily, considering that it would touch upon a broader fundamental issue relating to the nature of human consciousness and its potential boundaries. In that context, some of the relevant questions for open consideration would be: Is there an aspect of consciousness that might extend into the physical world? And if so, to what degree? Where exactly do mind and matter intersect with each other?

Phenomena hinting at a possible influence of mind upon matter have reportedly been witnessed and described in many cultures throughout the course of human history (Auerbach, 1996; Heath, 2011). Some of the earliest known accounts relate to apparent displays of lithobolia (or "stone-throwing") reported in the 17th and 18th centuries, in which numerous stones or pebbles were said to have spontaneously propelled themselves through the air in the presence of human witnesses (Davidson \& Duffin, 2012; Puhle, 1999). Reports were particularly profuse during the height of the Spiritualism movement in the late 19th century, when various raps, knocks, table tippings and levitations, materializations, and other seemingly anomalous physical events were described in the context of séance sittings held in the presence of reputed physical mediums (Braude, 1991, Ch. 2; Carrington, 1920; Crookes, 1889; Gregory, 1985; Nisbet, 1973; Pilkington, 2006; Weaver, 2015). While their numbers seemingly began to decline from the 2oth century onward, there remained to be a fair amount of spontaneous PK reports that surfaced from time to time in various situational contexts (Alvarado, 2006; Biondi, 2009; Rhine, 1963; Wright, 1998), most often in cases of ostensible poltergeist and haunt phenomena (Auerbach, 1996; Carrington \& Fodor, 1951; Cornell, 2002; Flammarion, 1924; Gauld \& Cornell, 1979; Huesmann \& Schriever, 1989; Puhle, 1999; Rogo, 1986; Roll, 1977, 1983). Mild success at intentionally producing overt PK 
phenomena was also said to have been occasionally achieved in the context of sitter groups partly modeled after the earlier séance sittings (Batcheldor, 1966; Brookes-Smith, 1973; Brookes-Smith \& Hunt, 1970; Owen \& Sparrow, 1976; Pilkington, 2006; Ullman, 2001).

Although such reports of spontaneous PK-related phenomena often seemed quite intriguing, their evidential value is somewhat limited by the fact that they were often based primarily on anecdotal observations made under conditions that were not always ideally controlled. On this basis, questions could be raised about whether the phenomena did reflect genuinely anomalous physical effects, or whether they were simply spurious demonstrations artificially produced through trickery, misperception, or unrecognized ordinary physical factors. As a means of addressing this issue, attempts at producing PK phenomena in the laboratory became one focus of the development of experimental parapsychology in the 2oth century.

Early laboratory experiments on PK were partly inspired by gambling and gaming scenarios, involving attempts to mentally affect rolling six-sided dice (Pratt, 1960; Rhine, 1970). Starting with the efforts initiated by J. B. Rhine and his colleagues at Duke University in the early 1930s, there were nearly 150 experimental PK tests with dice conducted over a 52-year period spanning from 1935 to 1987 , involving 2,569 volunteer participants attempting to willfully affect nearly 2.6 million dice rolls. Collective evaluation of these experiments via metaanalysis reveals a small but highly significant overall effect (Stouffer's $Z=18.2, p<10^{-70}$, with an odds ratio greater than a billion to one. In stark comparison, the overall result for 31 control tests-amounting to 153,288 dice rolls - was entirely consistent with chance (Stouffer's $Z$ $=0.18, p=.429$ ), with an odds ratio of only about two to one (Radin \& Ferrari, 1991). These findings seemed to be in line with a PK effect occurring on a small (possibly microscopic) scale.

To date, the most extensive number of experiments on PK have similarly entailed examinations of PK on the microscopic scale, involving attempts to mentallyinfluence the output of electronic, binary-bit random number generators (RNGs, also sometimes synonymously referred to as random event generators, or REGs). ${ }^{1}$ As with other experimental areas related to the study of ostensible psychic (or psi) phenomena, micro-PK research with RNGs can generally be divided into two lines: The first 
line involves proof-oriented research, which is mainly concerned with the progressive accumulation and statistical evaluation of RNG data from controlled experiments specifically designed with the intent to detect the presence of micro-PK effects on RNG output. In short, it involves research geared toward the effort to build a database of replicable evidence (or "proof") for seriously considering the existence of PK.

The second line relates to process-oriented research, which mainly focuses on conducting and evaluating exploratory experiments that are purposely designed to systematically vary certain test conditions in order to search for (and eventually identify, through consistent replications) any physical, biological, or psychological factors which might have a role in enhancing or modulating PK effects. In short, it involves research that is geared toward learning more about the processes which might underlie PK functioning, or (in simpler terms) learning more about "how PK might work."

After eight decades of research, one might wonder: What have we learned so far from these experiments, and in what directions should RNG-PK research perhaps be focused in the future? To help address these questions, a general review is provided here of some of the notable proof-oriented and process-oriented findings which have emerged from RNG-based micro-PK experiments to date. For the sake of keeping this review to a manageable length, the intent here is not to give an exhaustive treatment of all the available findings along these lines, but rather to focus primarily on findings which have emerged from certain subsets of related studies on a given proof-oriented or process-oriented topic. The aim is to try and begin gaining some preliminary insight on the following:

- What kinds of evidence for PK have amassed from prooforiented RNG testing?

-Are they any kinds of physical, biological, and psychological factors which might be conducive to successful micro-PK performance?

It is hoped that this review will be particularly helpful in orienting novice investigators and cross-disciplinary researchers who may be considering research along any of these lines, as well as in offering 
initial guidance for pursuing further replications and exploratory research related to findings which seem potentially fruitful.

\section{PROOF-ORIENTED RESEARCH: WHAT EVIDENCE IS THERE FOR PK FROM RNG TESTS?}

\section{Early PK Research by H. Schmidt}

The RNG revolution in parapsychology largely began in the 1970s when Helmut Schmidt, a physicist at Boeing Laboratories in Seattle, Washington, had designed and introduced a compact, electronic RNG device that could be used for applications in psi testing $(\mathrm{H}$. Schmidt, 1970b, 1970c). In its simplest form, the device based its randomness on natural radioactive particle decay from a sample of the isotope Strontium-90, which was monitored through a Geiger-Müeller tube. The tube was interfaced to a high-frequency electronic switch that rapidly oscillated between two equally possible binary state outcomes (" 1 " and "o"), with each having a $50 \%$ probability of occurrence. The decay emission of a particle happened at intervals that were inherently unpredictable, and whenever an emitted particle was detected by the tube the switch was stopped and the binary state outcome (indicated by the resulting momentary position of the switch) was registered on an electromechanical counter and recorded on a strip of paper tape. After a delay lasting about one-tenth of a second, the switch was reset and it continued its rapid oscillating behavior until the next emitted particle was detected. The device continually repeated this process for a pre-defined number of trials, thereby facilitating the generation of a random binary sequence. ${ }^{2}$

Schmidt initially utilized an RNG of this type (with the GeigerMüeller tube interfaced to a four-choice switch) to conduct preliminary studies of clairvoyance and precognition (H. Schmidt, 1971b, 1990), but he soon came to realize that the significant results he obtained in those studies could potentially be explained not only by psychic perception of the outcome, but also by exerting a psychokinetic influence on the RNG output. This prompted him to explore the PK hypothesis in a pilot study comprising two individual test series with the binary-bit RNG device (H. Schmidt, 1970a). 
During each of the test sessions in the preliminary series, a participant was seated before a visual feedback display consisting of a circular row of small lamps. The lamps were lit sequentially, one at a time, moving in either the clockwise or counterclockwise direction, with the direction being randomly determined at any given moment by the output of the RNG (to which the display was externally interfaced). The participant was tasked with trying to willfully make the lamps consistently light up in one particular direction for as long as possible, which could be hypothetically achieved by mentally influencing the RNG output to produce more "1" bits than would be expected by chance. Rather surprisingly, the overall result ("Prelim" in Figure 1) was suggestive of an influence in the direction opposite to that of the participants' willful intentions, with a success rate of $49.5 \%$ being obtained (13,695 successes out of 27,648 total bits; $z=-1.55, p=.06)$.

Results Summary: Early RNG-PK Studies by Helmut Schmidt

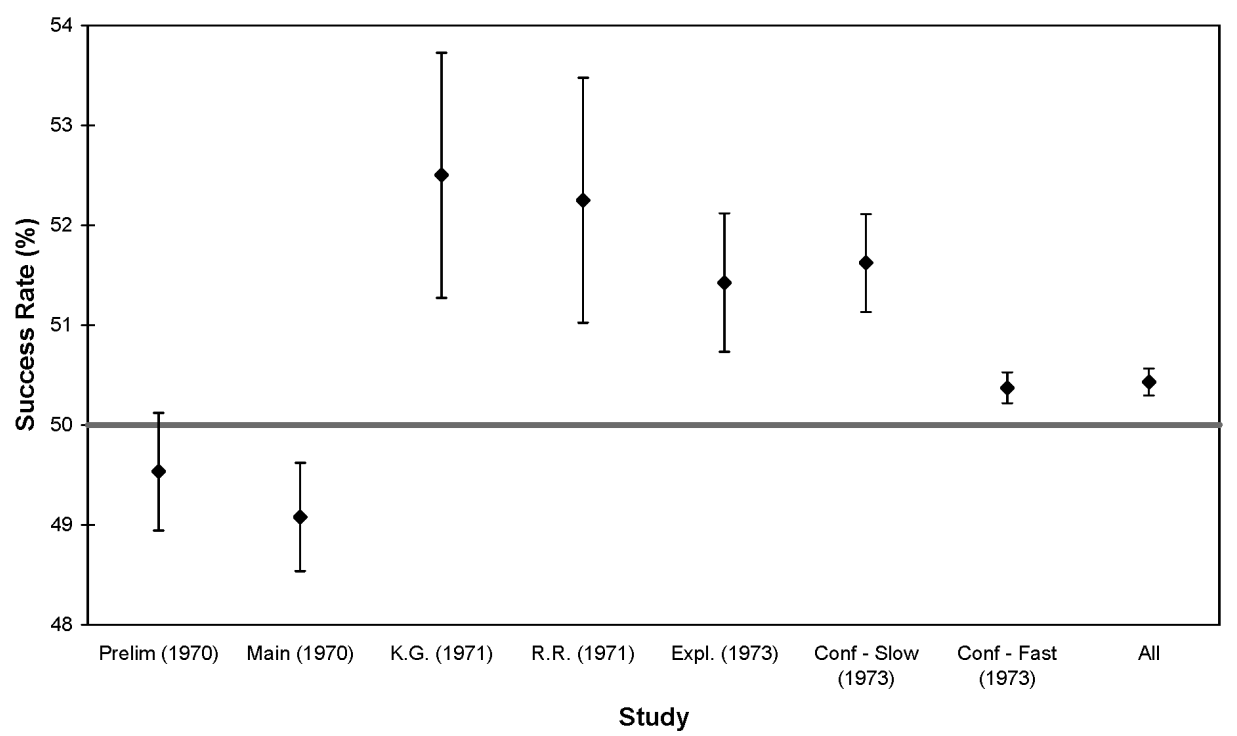

Figure 1. Graphical summary of the results obtained in the early RNG-based PK experiments conducted by $\mathrm{H}$. Schmidt (1970a, 1971a, 1973), expressed in terms of success rate (black diamonds) and associated $95 \%$ confidence intervals (thin vertical bars). The thick grey horizontal line at $50 \%$ represents the mean chance expected success rate. See text for study details. 
On the basis of this preliminary finding, H. Schmidt (1970a) predicted that the overall result in the second (main) series would also exhibit a negative deviation, and to try and encourage this tendency toward PK-missing he asked several participants who had exhibited a negative-scoring tendency in the preliminary series to again take part in the main series, and he further asked some of them to personally approach their second testing sessions with feelings of pessimism and discouragement. The result was indeed strongly in the predicted direction ("Main" in Figure 1), with a $49.1 \%$ success rate being obtained $(16,082$ successes out of 32,768 bits; $z=-3 \cdot 34, p=.0004)$.

To exclude the possibility that the significant results in these two series were due to a bias caused by a malfunction in the RNG, Schmidt also performed various control sessions in which the RNG was allowed to run unobserved for prolonged periods on separate days. The overall results, involving four million binary numbers, were found to be consistent with chance.

Notable results were also obtained in a second PK study that $\mathrm{H}$. Schmidt (1971a) conducted with two selected participants. The first participant was "an aggressively outgoing American girl” (p. 758) known as K. G., who exhibited a tendency to influence the RNG output in a positive direction in an early informal test series conducted with her. This tendency persisted in a formal confirmatory test, with a $52.5 \%$ success rate being obtained ( 3,360 successes out of 6,400 bits; $z=4.00$, $p=3.17 \times 10^{-5}$; "K.G." in Figure 1). The second participant was a South American psi researcher known as R. R., who exhibited a negativegoing tendency in his informal test series. This tendency was also maintained in his formal confirmatory test, which resulted in a success rate of $47.8 \%$ (3,056 successes out of 6,400 bits; $z=-3.60, p=.0002$; "R.R." in Figure 1$)$.

To see if the PK effects observed in these previous test series could possibly be enhanced with a greater number of bits, H. Schmidt (1973) conducted a third study with a newly constructed RNG that could generate binary bits at a higher rate of speed (up to 1,000 bits per second) by sampling electronic noise. Feedback on the RNG's output could be provided to participants either through auditory means (by relaying the output sequence as a series of clicking noises played into their ears via headphones) or visual means (by representing the 
output sequence on a running marker chart, where each individual bit outcome was registered by a deflection of the marking pen in one particular direction), and the PK task involved the participant trying to willfully favor one particular side of the feedback relay over the other (e.g., by trying to make more of the clicks play in the right ear rather than the left, or trying to make the pen deflect more often to the right side of the chart as opposed to the left).

Two test series were conducted using each of the two feedback types: The first (exploratory) series, conducted with four selected participants (one of whom was Schmidt himself), was in line with an intended deviation in the positive direction, with a $51.4 \%$ success rate $\left(10,285\right.$ successes out of 20,000 bits; $z=4.03, p=2.79 \times 10^{-5}$; "Expl." in Figure 1), with both types of feedback being effective to a roughly equal degree.

To see if PK performance might be affected in any way by varying the rate of bit generation, the second (confirmatory) series examined success rates at both high (300 bits/second) and low (30 bits/second) speeds. To make the two-bit generation rates appear overtly similar to each other from the participant's viewpoint, the individual test trials in each instance were set to span the same lengths of time. Both bit rates were found to produce positive results, with the slow bit rate resulting in a higher success rate $\left(51.6 \% ; z=6.49, p=4.31 \times 10^{-11}\right.$; "Conf. - Slow" in Figure 1) than the fast bit rate (50.3\%; $z=4.71, p=1.24 \times 10^{-6}$; "Conf. - Fast" in Figure 1). The two types of feedback were again found to be equally effective in this series, as well. (Further discussion of the effect of varying bit rates will be made in a later subsection of this paper.)

When combined ("All" in Figure 1), the results across all of these early PK studies by $\mathrm{H}$. Schmidt are highly significant (Stouffer's $Z=$ $4.06, p=2.46 \times 10^{-5}$ ), with an average success rate of about $50.3 \%$ and an associated odds ratio of about 40,650 to one. (For a broader readable overview of these early PK studies, see H. Schmidt, 1974a.)

\section{PEAR "Benchmark" RNG Program}

One of the most extensive and well-known efforts to experimentally study PK using RNGs was conducted by the staff of the Princeton Engineering Anomalies Research (PEAR) Laboratory, which was active in the School of Engineering and Applied Science at Princeton University 
from 1979 to 2007 (Dobyns, 2015; Dunne \& Jahn, 1995; Jahn, 1982; Jahn \& Dunne, 1987, 2005, 2011; Nelson et al., 1986). The effort consisted of an experimental program that was run over the course of a 12-year period (Jahn et al., 1997), involving 91 volunteer participants who each made multiple attem pts to mentally affect the output of PEAR's customdesigned "benchmark" RNG, a benchtop microelectronic device which, as succinctly described by Dobyns (2015), based its random binary bit generation on

... the trickle current in a diode (a solid state rectifier) that was being forced to carry current in the "wrong" direction. Since such currents depend on the ability of electrons to cross an energetically forbidden region by quantum tunneling, this current is every bit as much a quantum-mechanical random phenomenon as a radioactive decay. (Dobyns, 2015, p. 220)

The device was typically programmed to generate a sequence of trial values of 200 random binary bits each, with each individual trial value reflecting the total number of " 1 " bits which resulted from that trial; the theoretically-expected mean value was 100, with a standard deviation of 7.071. An extensive series of calibrations performed with the RNG, amounting to just over 5.8 million trials total, had generally indicated that the device's bit output sufficiently conformed to these expected values over the long term (Jahn et al., 1997; Nelson et al., 1989).

In a typical experimental PK test run, a participant was seated across from the benchmark RNG (with no physical contact being made between the two) and asked to try and mentally influence its trial bit outcomes across three separate experimental conditions:

$\mathrm{HI}$ - the participant's influence was aimed at having the RNG generate more trial bit outcomes with high values (i.e., generating more "1"-bit sums that are above 100), or increasing the overall trial mean of the test run;

LO - being the opposite of "HI," the aim was at having the RNG generate more trial bit outcomes with low values (i.e., generating more "1"-bit sums below 100), or decreasing the overall trial mean of the run;

$B L$ - the participant's influence is simply aimed at having the RNG generate a nominal baseline, with trial bit values that equal 
(or come in close range to) the theoretically-expected mean value. Equal amounts of data were gathered across the three conditions, with test runs ranging from 1,000 to 5,000 trials per condition.

As a convenient form of visual assessment, the statistical results of the test run were often graphically plotted as a cumulative measure of the degree of deviation away from the mean-expected value (often short-handed to "cumulative deviation"); a generic illustrative example is shown in Figure 2, representing the graphical display of a sequence of 3,600 trials generated by an RNG running unobserved, with no attempt at mental influence presumably being applied. Mean chance expectation (MCE) is represented in the graph by the level horizontal line at zero, while increases along the $\mathrm{Y}$-axis represent a deviating tendency toward higher trial outcome values (above MCE), and decreases represent a tendency toward lower trial values (below MCE).

Expected Look of RNG Data ("Random Walk")

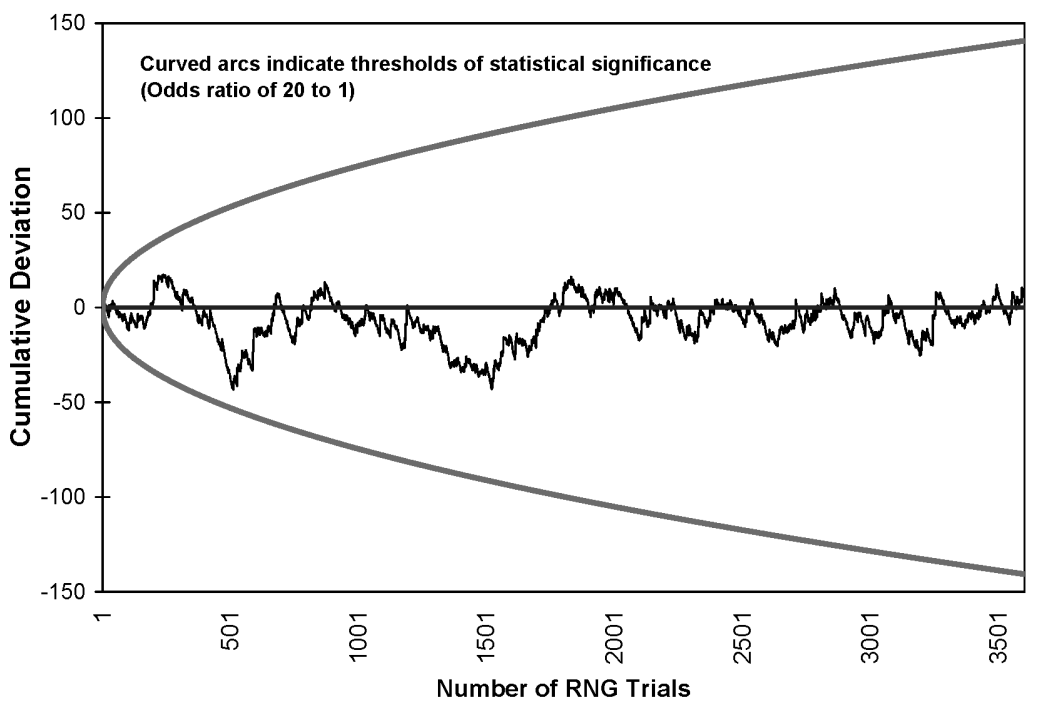

Figure 2. Illustrative example of a cumulative deviation plot, displaying the ideal look of a nominally random sequence being generated by an RNG while producing an output of 3,600 individual trials. The dark horizontal line at zero represents mean chance expectation (MCE), and the bold grey arcs represent the thresholds of statistical significance at $p=.05$ (above MCE) and $p=.95$ (below MCE) as trials accumulate. 
The bold-curved arcs indicate the threshold of statistical significance at both the $p=.05$ (positive) and $p=.95$ (negative) levels as the trial data gradually accumulate over time. The actual trial output sequence of the RNG is represented by the jagged line, which in this example exhibits a fairly ideal random walk (sometimes colloquially referred to as a "drunkard's walk") that maintains a level degree of random fluctuation around MCE. ${ }^{3}$

A graphical summary of all the benchmark RNG data collected by PEAR over the course of its 12-year program (amounting to nearly 2.5 million trials total) can be seen in Figure 3. The graph shows the data divided up into the respective cumulative deviation plots for each of the three mental influence conditions ( $\mathrm{HI}, \mathrm{LO}, \& \mathrm{BL})$, and it can be seen that for each condition a notable deviation from MCE was observed in conjunction with the influential aim of the participants. Of particular

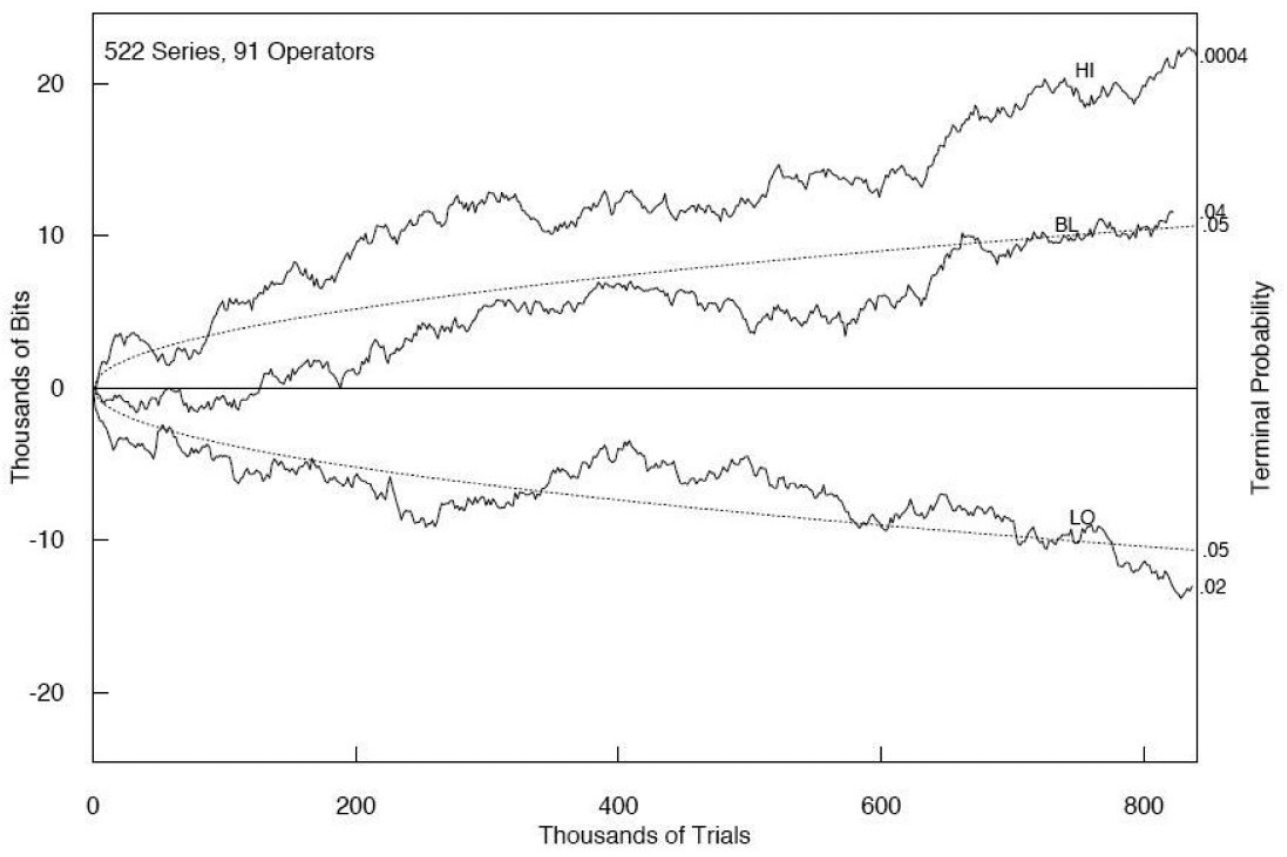

Figure 3. Graphical summary of the experimental data collected by PEAR over the course of its 12-year "benchmark" RNG program (Jahn et al., 1997), with the cumulative deviation plots for each of the three test conditions (HI, LO, \& BL) being displayed. Adapted from figure 2 of Jahn et al. (1997). 
note, the observed deviations for the $\mathrm{HI}(Z=3.37, p=.0004)$ and the $\mathrm{LO}(Z=-2.02, p=.022)$ conditions were each independently significant, and the formal test of the PK hypothesis-predicted in the form of a notable overall difference between the $\mathrm{HI}$ and LO conditions-yielded a highly significant effect $\left(Z_{\text {diff }}=3.81, p=6.99 \times 10^{-5}\right)$ with an odds ratio of about 14,380 to one.

\section{Mind/Machine Interaction Consortium Replication Study}

Partly as a means of further following up on the PEAR work on a broader scale, a "Mind/Machine Interaction Consortium" was formed in 1996 among three laboratories—the Institut für Grenzgebiete der Psychologie und Psychohygiene(IGPP) in Freiburg, Germany; the JustusLiebig Universität's Center for Psychobiology and Behavioral Medicine in Giessen, Germany; and PEAR - with the intent of attempting to directly replicate the experimental methodology and main findings of the PEAR benchmark program as closely as possible, using second-generation RNG technology (Jahn et al., 2000). The three-year effort called for each laboratory to conduct 250 experimental PK sessions in which 227 volunteer participants were asked to try and mentally influence the same particular type of compact RNG (designed to utilize thermal noise in resistors as its source of randomness), with equal proportions (250,000 trials) of data being collected for each of the three influence conditions $(\mathrm{HI}, \mathrm{LO}, \& \mathrm{BL})$. The measure pre-specified in advance to serve as the formal test for replication was the significant overall difference between the $\mathrm{HI}$ and LO conditions that had been observed in the PEAR results. Automated calibration sessions which followed the experimental ones, amounting to just over 3.1 million trials total, generally indicated that the RNGs used by the three laboratories had remained sufficiently random as expected over the course of the replication.

The main experimental results of the Consortium replication (Figure 4), when graphically summarized and compared against the original PEAR benchmark findings in the manner depicted by Radin (2006, p. 156), seemed to indicate two things:

1. The replication z-scores for the HI, LO, and BL conditions (dark circles in Figure 4) seemed to generally reflect the same directional patterns as those observed for these three conditions in the PEAR benchmark program (shaded bars). This seemed to suggest that there 


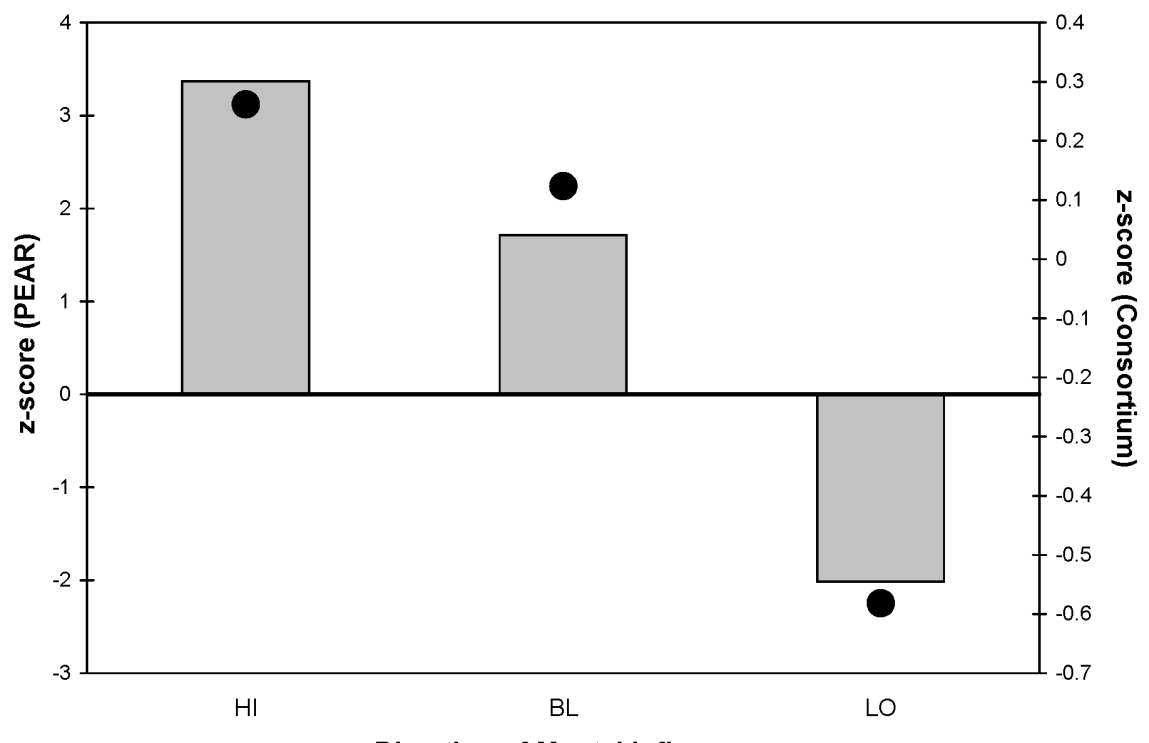

Direction of Mental Influence

Figure 4. Graphical summary and relative comparison of the combined experimental RNG results from the "Mind/Machine Interaction Consortium" replication study (Jahn et al., 2000; dark circles) with the results obtained in the 12-year PEAR "benchmark" RNG program (Jahn et al., 1997; shaded bars). Adapted from Radin (2006, figure 9-4).

was a consistent tendency for the experimental RNG data in each case to become shifted in a manner in line with the influential aims of the participants.

2. None of the replication $z$-scores managed to attain the minimal standard score for statistical significance (i.e., $z>1.65$ ), and when examined in relative comparison with each other, the replication scores are notably smaller than the PEAR benchmark z-scores by about one order of magnitude.

Combined together, the data from all three laboratories resulted in a nonsignificant $\mathrm{HI}-\mathrm{LO}$ difference $\left(Z_{\text {diff }}=0.59, p=.278\right)$ that was one order of magnitude smaller than the previously observed PEAR difference. But while this result did not offer clear evidence of an anomaly, several secondary analyses planned in advance to look for certain structural patterns in the replication data that had been previously found in the PEAR benchmark data (such as serial position effects, discussed in a later subsection) did collectively produce a result suggestive of a lesser anomaly in the data $(p=.022)$. 


\section{RNG Meta-Analyses: 1985-2006}

In addition to those conducted by $\mathrm{H}$. Schmidt, PEAR, and the Consortium, many other RNG-based PK experiments have been conducted by parapsychologists since the 1960s. When collectively taken into account as a whole, do the data from these experiments exhibit evidence of a notable deviation from expected randomness over time? To find out, five meta-analyses of the accumulating RNGPK database have been progressively conducted and reported over the past several decades. The first was conducted and reported by Radin et al. (1986), who surveyed 381 experimental PK tests conducted by 38 different researchers over a 15 -year period spanning from 1969 (when the pioneering work by $\mathrm{H}$. Schmidt was first reported) to 1984 . Using a simple result counting method, they found that 71 of these experiments had reportedly been successful at the $p<.05$ level, whereas only 19.05 would have been expected by chance. Such an outcome has an associated odds ratio greater than a billion to one (binomial $p<5.4 \times 10^{-43}$ ).

The second analysis was conducted and reported by Radin and Nelson (1989), who examined 597 experimental and 237 control sessions conducted by 68 different researchers over a broader period of 28 years, from 1959 to 1987 . When expressed in terms of success rate, ${ }^{4}$ the combined, quality-weighted result for the experimental sessions is quite small (50.016\%, "RN 1989" in Figure 5), reflecting only a narrow fractional shift above the expected mean value. But despite its small size, the result is highly significant, reflecting a six-sigma deviation from chance expectation with an associated odds ratio of about 30 billion to one $\left(Z=6.53, p=3.30 \times 10^{-11}\right)$. In contrast, the result for the control sessions was entirely consistent with chance. (For a broader readable summary, see Ch. 8 of Radin, 1997.)

Following up on their initial finding fourteen years later, Radin and Nelson (2003) expanded their database for the third analysis to 515 experiments conducted by 91 researchers over a period of 41 years, from 1959 to $2000.5^{5}$ The success rate associated with the weighted overall experimental result was again quite small in magnitude $50.005 \%$, "RN 2003 " in Figure 5), but still highly significant, with odds of about 14,300 to one $\left(Z=3.81, p=6.95 \times 10^{-5}\right)$.

The fourth analysis, conducted by Radin (2006), had further 


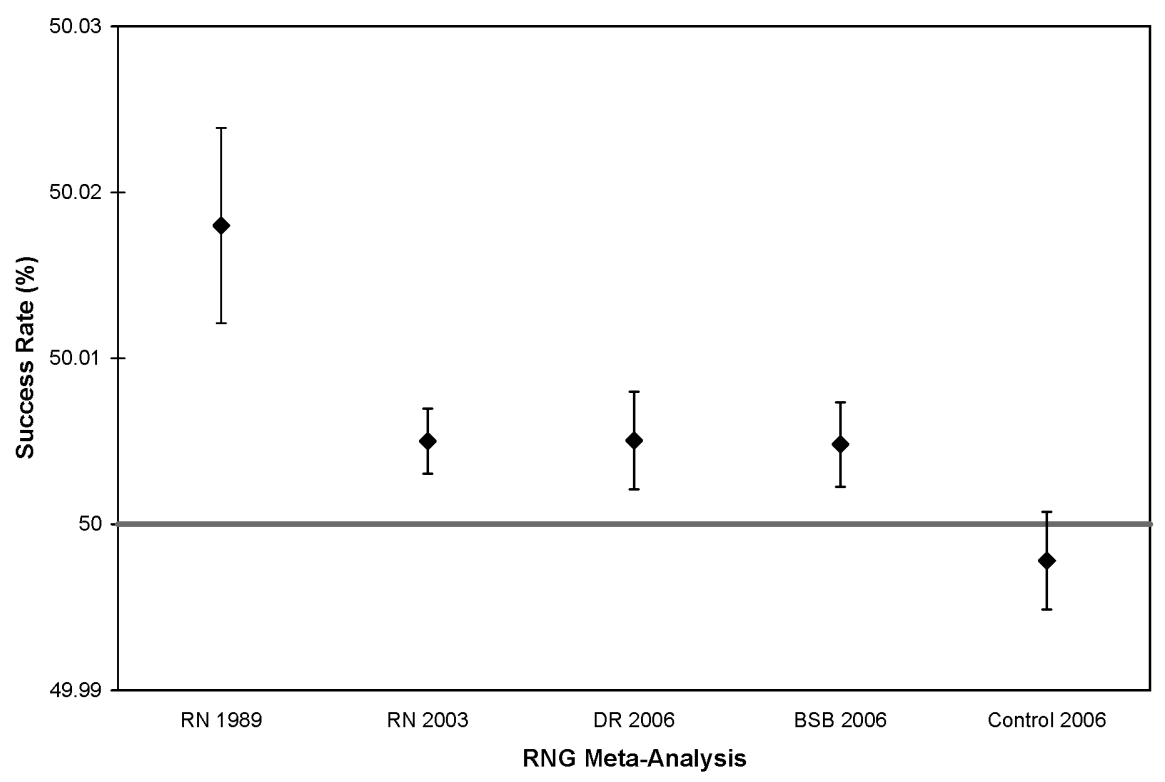

Figure 5. Graphical summary of the success rates (black diamonds) and associated 95\% confidence intervals (thin vertical bars) from four RNG-PK metaanalyses reported by Radin \& Nelson in 1989 ("RN 1989") and 2003 ("RN 2003"), Radin (2006; "DR 2006"), and Bösch et al. (2006a, "BSB 2006"), along with the control dataset analyzed by Bösch et al. (2006a, "Control 2006") for comparison. The thick grey horizontal line at $50 \%$ represents the mean chance expected success rate. See text for study details.

updated the database to 490 experiments covering the 47 -year period from 1959 to $2006,{ }^{6}$ and yielded a comparable success rate $\left(50.006 \%,{ }^{7}\right.$ "DR2006" in Figure 5). This weighted result also was highly significant, with odds of about 39,000 to one $\left(Z=4.05, p=2.56 \times 10^{-5}\right)$.

The fifth and most recent meta-analysis by Bösch et al. (2006a) had independently examined 380 experimental and 137 control sessions conducted by 59 researchers from the 1960 s up to 2004 . Conservatively, the combined success rate (50.004\%, "BSB2006" in Figure 5) for the experimental sessions was comparable to those obtained in the previous two analyses, with an odds ratio of about 147 to one $(Z=2.47, p=.0068)$.

One contributing source to this more modest result appeared to be the three largest experiments contained in the test database, which exhibited significant directional trends of deviation that were opposite 
to prediction (i.e., they exhibited significant shifts from expected randomness in the negative direction, rather than in the often-predicted positive direction; more will be said about these experiments in a later subsection on RNG bit rate). When these three largest experiments were excluded, the combined experimental success rate increased to $50.029 \%$, with odds of about 44,300 to one $\left(Z=4.08, p=2.25 \times 10^{-5}\right)$. In marked contrast, the control success rate was very close to chance (49.998\%, "Control 2006" in Figure 5), with odds of only 14 to one $(Z=$ $-1.51, p=.13) .^{8}$

\section{Retro-PK Studies}

While most RNG tests for PK have involved participants attempting to mentally influence a sequence of random numbers being generated by the RNG in real-time (i.e., at the same present moment), a small proportion of them were also designed with the purpose of determining whether PK could possibly act retroactively - that is, to determine whether participants could possibly influence random number sequences that have already been generated and recorded some time before the PK test begins.

One of the earliest studies of retroactive PK (or retro-PK, for short) was conducted by $\mathrm{H}$. Schmidt (1976), in which he initially programmed an RNG to automatically generate many individual sets of random number sequence outputs (each composed of 201 binary numbers) that were registered as audible clicks and pre-recorded on to audio cassette tape. The original tape was then duplicated and the original was kept secure by Schmidt, while the copy was given to psychic claimant Sean Lalsingh Harribance, who was asked to take it home and try to influence the clicks while listening to them being played back on a tape player. (When played back, the clicks were randomly presented at high- or low-volume, and Harribance's task was to try to increase the number of low-sounding clicks.) Subsequent counting of the clicks recorded on the original tape (done only after Harribance had fully listened to the copy, with the reasoning for this being based on the observational approach, described below) did indeed indicate a modest increase in the number of low clicks ( $55.5 \%$ success rate, $z=2.23, p=.013$ ), in line with the intended goal.

In three other exploratory tests conducted in a laboratory setting, 
the pre-recorded random number sequences were coupled to the left/ right movement of an instrument needle, and Harribance was tasked with trying to make the needle swing more often to the right. Though again in line with the intended goal, the result was only suggestive at best $(53.6 \%, z=1.44, p=.075)$.

When combined, these initial exploratory tests with Harribance resulted in a significant overall success rate $(54.6 \%, Z=2.60, p=.0046)$, with an odds ratio of about 210 to one. These (along with a few other pilot tests with promising results) had motivated H. Schmidt (1976) to pursue a series of three formal retro-PK experiments of varying complexity, each of which produced significant results with approximate odds ratios ranging from 45 to 2,000 to one (i.e., $p$-values ranging from .021 to .0005).

In addition to this early study by $\mathrm{H}$. Schmidt, 23 other retroPK experiments using various kinds of targets were conducted and reported by nine different researchers over an 18 -year period spanning 1975 to 1993 . Upon being combined, Bierman (1998) initially found that these 26 experiments collectively amount to a highly significant outcome $\left(Z=5.31, p=5.49 \times 10^{-8}\right)$ with an associated odds ratio of about 18 million to one. If Bierman's analysis is further updated to the present and is limited solely to experiments using data pre-recorded from RNGs (see table in the Appendix), one finds that there have been a total of 42 experiments reported from 1975 to 2021, which continue to exhibit a highly significant overall result exceeding $\operatorname{six} \operatorname{sigma}(Z=6.82$, $p=4.57 \times 10^{-12}$ ) with nearly a trillion to one odds ratio.

If these significant results can be taken as being reflective of a retro-PK effect, then one may be led to wonder: How might the mind be able to influence random data that has already been generated and recorded prior to the PK test? One possibility is based on the assumption that PK may somehow be capable of operating in a retrocausal fashion (H. Schmidt, 1993b) — that is, it may somehow be capable of working in a manner opposite to the conventional temporal direction of cause and effect, such that the directional flow would be reversed to where an effect seems to precede (rather than follow) its cause. Another way of looking at this is that PK would have to somehow act backwards in time in order to influence the data at the moment it was being generated in the past, prior to the PK test. 
An alternate possibility, which circumvents this assumption of retrocausality, has its basis in observational theory (Houtkooper, 2002a, 2006b; Millar, 1978; H. Schmidt, 1975, 1976; Walker, 1975, 1984, 2000), an approach based on certain interpretations of quantum physics which may be succinctly conceptualized in a single statement as: "[T]he act of observation by a motivated observer of an event with a quantum mechanically uncertain outcome influences that outcome" (Houtkooper, 2002a, p. 172). This alternate viewpoint begins with the recognition that an RNG's random binary number sequences are derived from sampling the quantum-based activity of subatomic particles, which is inherently probabilistic. Prior to the PK test, these sequences are automatically generated and recorded when no one is present to directly observe them, which presumably leaves their binary outcomes undetermined in a state of quantum superposition (where it remains equally likely that a particular binary outcome could result in a " 1 " or a "O"). It is not until the sequences are directly observed later on by the participant (upon being played back during the PK test) that their outcomes are actually determined (and presumably influenced in the process of being observed) by a motivated observer (i.e., the participant, who is aiming for a greater proportion of one particular binary outcome over the other). Thus, according to this observational approach, retro-PK might simply be viewed as being a kind of "delayed" PK effect (Houtkooper, 2006b; H. Schmidt, 1975, 1976, 1982, 1987a; Stapp, 1994; Walker, 1975, 1984).

This observational approach can help one to understand the rationale behind the procedure employed in H. Schmidt's (1976) earliest exploratory test, where the analysis of the original tape was conducted after Harribance listened to, and attempted to influence, the copy: Because the two tapes are exact duplicates of each other, they contain the same binary bit data stemming from the same source (the RNG), and from the perspective of quantum theory can perhaps be thought of as representing an entangled system. Initially, one would presume that, prior to being observed, the data contained on the two tapes would be in a state of quantum superposition. But upon being observed by Harribance (at the time he is listening to them), the binary bit outcomes for the data contained on the copy tape would presumably become determined (and influenced via PK). And presumably, since the 
data contained on the two tapes are entangled, the effect of Harribance observing and influencing the data on the copy tape should then also (nonlocally) affect the data contained on the original tape, such that its binary bit outcomes would become determined and influenced, as well. This would then facilitate an analysis of the data from the original tape as a means of evaluating the results of the PK test. Such a procedure would also happen to contain a convenient preventive measure against data tampering, since the original tape remains secured away by the experimenter during the test and is not observed or handled by anyone.

\section{Field RNG Studies}

The significant effects upon RNG output observed in laboratory PK experiments may lead some to raise the ecologically relevant question: To what extent might these effects apply to more naturalistic settings? As a preliminary means of finding out, the PEAR Laboratory began developing a more compact and portable electronic noise-based RNG system in the 1990s that could be deployed out in the field (Bradish et al., 1998), and these "field RNGs" were placed in close proximity to various kinds of social group venues and environments that initially included conferences, workshops, religious ceremonies, council meetings, and a geographic site where many witnesses reported seeing ostensibly anomalous atmospheric light phenomena. In most of these instances, the field RNG system was positioned unobtrusively in the background and programmed to continuously collect binary bit data in a silent fashion, with few or none of the group members being aware of its presence. An important implication of the latter is that unlike laboratory tests, these field studies of PK would not entail the conscious exertion of mental will-rather, any influencing effects upon the field RNGs would presumably be manifesting on the subconscious or unconscious level.

Upon being statistically combined, the field RNG data from these various group venues and environments had exhibited a significant departure from expected random behavior $(Z=3.54, p=.0002)$ that was very similar to the deviations from nominal randomness observed in laboratory PK experiments. In notable contrast, a matching set of field RNG data drawn from times when no social group activity was 
taking place at the venues and environments had resulted in an overall random outcome that was consistent with chance $(Z=0.07, p=.461)$ (Nelson et al., 1996).

A confirmatory study was subsequently conducted which utilized a conceptually similar set of group venues and environments that included conferences, workshops, rituals, music amd theater performances, and tours of geographic sites with historical or cultural significance-all of which were predicted to exhibit a significant departure from nominal randomness in conjunction with the social group activity (Nelson et al., 1998). The prediction was confirmed by the resulting field RNG data ( $Z$ $\left.=4.59, p=2.20 \times 10^{-6}\right)$, with a combined odds ratio of about 450,000 to one. Significant results were also obtained in field RNG studies with conceptually similar designs that were independently conducted by several other researchers around the same time (Bierman, 1996; Broughton, 1999; Radin, 1997, Ch. 10; Radin et al., 1996; Rowe, 1998; Schwartz et al., 1997). (For a broader overview, see Nelson and Radin, 2003.)

Efforts to expand this field RNG work to a much larger scale were undertaken by Nelson (2001) and his colleagues in 1998 with the founding of the Global Consciousness Project (GCP), an international collaborative experiment designed to generally explore the hypothesis that significant departures from nominal randomness may possibly occur across multiple RNGs during moments when the attention and emotions of many people around the world are collectively engaged in unison, often in response to notable news events and activities that tend to draw widespread notice (Nelson, 2015, 2019; Nelson \& Bancel, 2011). At the heart of the experiment is an extensive, globalspanning network of RNGs, with each RNG node continually collecting 200-bit random binary samples every second of the day and sending its collected data over the Internet at regular intervals to a central server in Princeton, New Jersey, for archiving. Repeated testing of the experimental hypothesis is made in relation to the occurrence of an individually specified event or activity, with the data from the RNGs in the network being statistically combined and examined over the course of a defined period of time (typically a few hours or more) surrounding that event or activity. Some of the events and activities that have been examined include New Year's Eve celebrations, global meditations held 
on the International Day of Peace, U.S. Presidential elections, natural disasters (e.g., "Super Storm Sandy"), public ceremonies (e.g., the British Royal Wedding of Prince William and Catherine Middleton), and the devastating series of terrorist attacks on the World Trade Center and the Pentagon on September 11, 2001. Across a formal experimental database of 500 individual events and activities covering a roughly 17year timespan (from August 1998 up until the end of 2015), a small but highly significant overall effect amounting to a seven-sigma deviation from chance expectation $\left(Z=7.31, p=1.33 \times 10^{-13}\right)$ was found, with an associated odds ratio of about 4.1 trillion to one (Figure 6). (For a broader and readable overview, see Nelson, 2019.)

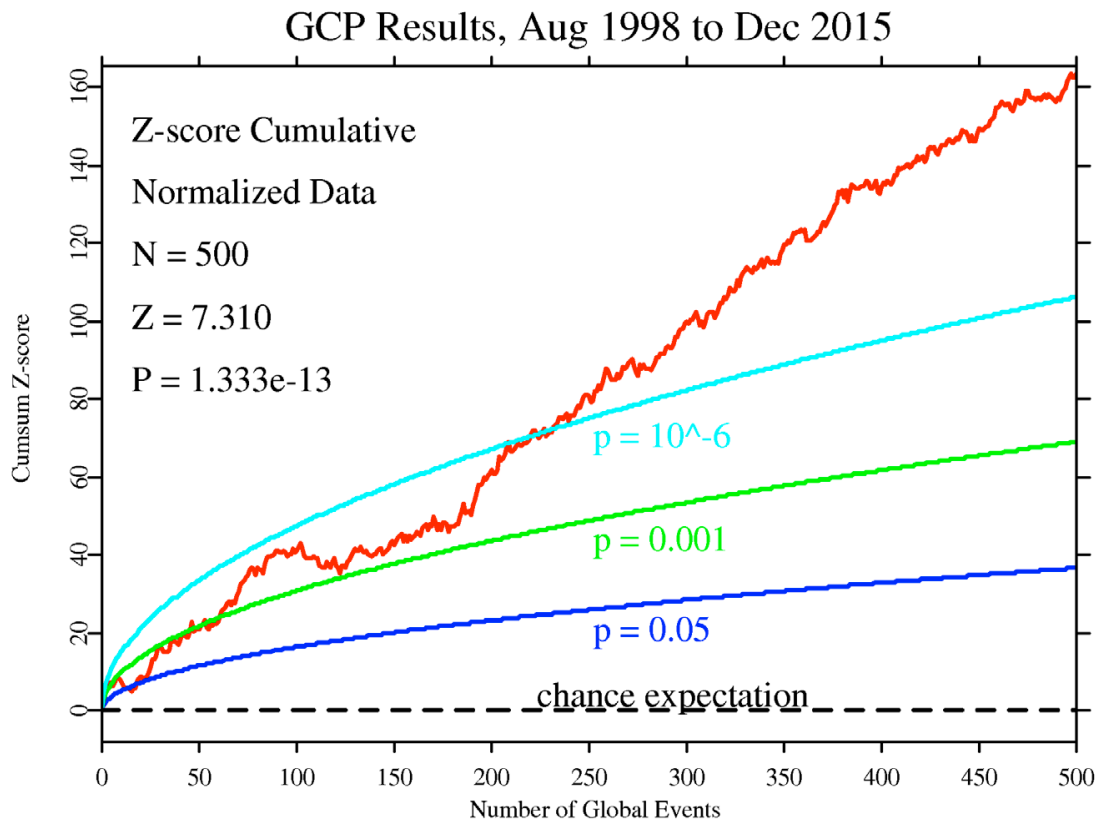

Figure 6. Graphical summary of the experimental results accumulated by the Global Consciousness Project with its worldwide network of RNGs over the course of its formal 500-event database from August 1998 to December 2015. Adapted from the "Results" page of the GCP website: https://www.globalmind.org/results.html 


\section{Other Notable Micro-PK Effects}

Serial Position (Decline/Recover) Effects. Some participants in experimental tests for psychic ability have previously exhibited a notable parabolic (widened U-shaped) pattern in their test results, where their scoring performance initially starts off high at the beginning of the test series, then decreases (or declines) toward the middle, and finally begins to increase (or recover) again near the end of the series. This pattern of scoring is often referred to as the serial position effect and has been observed in tests for extrasensory perception (ESP) (Pratt, 1949; J. B. Rhine, 1969) as well as in the early dice tests for PK (Pratt, 1949, p. 15; Reeves \& Rhine, 1943). It has also been observed in some PK tests using RNGs (Berger, 1988a; Houtkooper, 2002b), most notably in the benchmark experimental series conducted by the PEAR Laboratory (Dunne et al., 1994; Jahn \& Dunne, 2011, pp. 178-179; Nelson et al., 2000)(Figure 7), as well as in the subsequent Mind/Machine Interaction Consortium replication (Jahn et al., 2000).

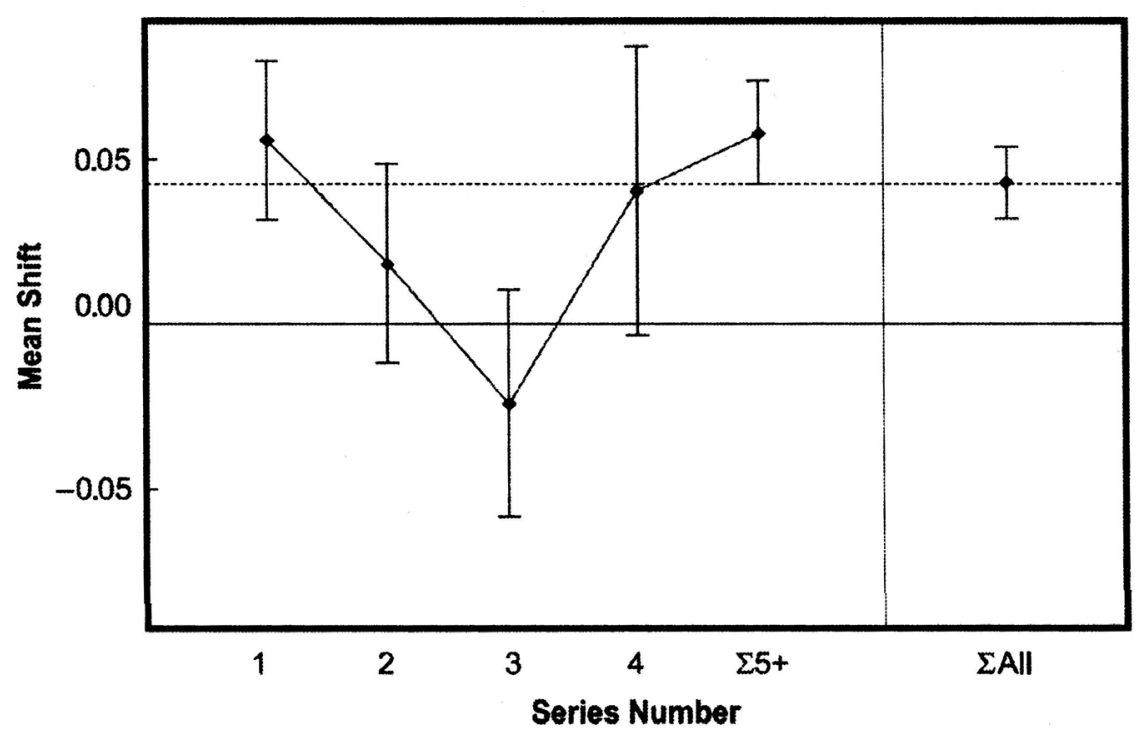

Figure 7. Graphical display of the serial position effect observed across the PEAR "benchmark" series of experiments, marked by a decline from Series 1 to 3 , followed by an inversion and subsequent increase from Series 3 to 5 . Adapted from Figure 2 of Dunne et al. (1994). 
There is even a hint that this parabolic pattern may be observable on the broader level of an entire experimental database: In statistically evaluating the results of 264 RNG-based PK experiments conducted from 1959 to 1987 , Bierman (2001, pp. 276-277) found a significant trend $(p<.03)$ occurring across their $z$-scores with time, which indicated that the scores first tended to modestly decrease from 1959 up until the early 1970s, and then began to rebound and gradually increase from the late 1970 up to 1987.

Something particularly notable about this parabolic pattern is that it is very similar to the kinds of serial position effects that have been observed in mainstream psychological experiments (Thompson, 1994), most often in studies of learning and memory (Reed, 2004, pp. 102-103). This has led some researchers to suspect that similar kinds of factors may perhaps be involved; as Pratt (1949) observed in this regard: "When we find this fundamental characteristic of normal behavior [i.e., the parabolic pattern of the serial position effect] in ESP and PK data, we have ample reason for inferring that we are dealing here with established psychological principles showing themselves in only less familiar forms" (p. 14). If that is the case, then this may offer one indication that PK (and psychic ability, in general) may not be so inherently different from other relatively "normal" forms of human behavior such as sensory perception and cognition.

Statistical Balancing Effects. In her own experimental studies of PK using RNGs, Pallikari (2016) uncovered possible indications of another kind of pattern in the data that she labeled "statistical balancing" (Pallikari-Viras, 1997, 1998), which is based on the idea that there is ". .. a tendency of nature to remain close to a preferred, undistorted, state of randomness, so that it reverts back to that undistorted state when pushed away from it by the anomalous effect of conscious intention" (Pallikari-Viras, 1997, p. 115). The pattern was observable across two sets of RNG data, labeled "intention" and "no-intention," which Pallikari had collected consecutively, back-to-back, in the course of a PK test session. As its label implies, the "intention" data were collected during a short period in which the participant made an effort to mentally influence the RNG output, with the intent of producing an excess of one binary outcome over the other. The "no-intention" data were then collected immediately afterward, during a period of equally short length in which 
the RNG was allowed to run on its own without the participant making any (conscious) effort to intentionally influence its output.

Upon being analyzed, the two datasets were found to exhibit a notably contrasting parallel: They both exhibited shifts away from nominal randomness, but in opposite directions-for instance, if the "intention" data were found to exhibit a shift in the positive direction, then the "no-intention" data would subsequently be found to exhibit a shift in the negative direction. These two opposed shifts appeared to roughly balance each other in such a way that when the two datasets were statistically averaged, their deviations from randomness ended up roughly canceling each other, yielding an overall outcome consistent with chance. Pallikari has observed such a balancing effect across the resulting z-scores for the "intention" and "no-intention" datasets in two of her five PK studies (Pallikari-Viras, 1998), and has found some suggestive indications that it can even extend to other statistical parameters of the underlying binomial distribution, such as the standard deviation, skew, and kurtosis (Pallikari-Viras, 1997); as well as to broader meta-analytical findings (Pallikari, 2016). Similar kinds of results which may also be suggestive of a balancing effect have been observed and reported in at least a handful of other studies (Bierman \& van Gelderen, 1994; Houtkooper, 2002b; Jahn \& Dunne, 1987, pp. 116-119; Radin, 1993b), suggesting that further efforts toward exploring such an effect should perhaps be made, in order to determine how common it might be.

Assuming that it does reflect a genuine effect, one possible implication of this opposing pattern across the two datasets is that when PK (or some other influencing factor) induces an RNG's output to shift away from its nominal random behavior during the "intention" period, a secondary rebound shift may subsequently occur in the "no-intention" period to counteract the PK shift and bring the RNG output back toward nominal randomness; such an implication would appear to be in line with a physical conservation principle, like those governing energy and mass. If that is the case, then this would begin to suggest that PK may not be so inconsistent with some of the known principles of physics.

Physical/Psychological Matrix Correlations. As a premise of his Model of Pragmatic Information, von Lucadou (1988a, 1995) proposed that psi phenomena can possibly be conceptualized not as direct causal 
interactions between mind and matter (as they might often be thought of in terms of classical mechanics), but as non-causal correlations akin to those exhibited by systems of entangled particles according to the predictions of quantum theory. From this conceptual perspective, micro-PK might be conceptualized not as a direct, mental "force"-like form of influence manifesting upon matter, but more like a subtle, non-causal (or nonlocal) correlation existing between the properties of the physical target and the mind of a human influencer. Based on such a concept, one might predict that rather than being directly reflected by cumulative deviations from expected randomness occurring in the RNG output (presumably being causally produced from the direct exertion of a "force"-like mental influence), the effects of micro-PK might be more indirectly detectable as a relatively high degree of correlation existing between a range of psychological variables associated with the human influencer, and a range of physical variables associated with the operation of the RNG. This led to the development of an analytical approach involving correlational matrix analysis (von Lucadou, 1987a, 1987b, 1988b, 1994; von Lucadou et al., 1987). Generally, in the most recent studies utilizing this approach (von Lucadou, 2006; Walach, 2014; Walach et al., 2020), the PK test proceeds as follows:

Participants watch a monitor screen displaying an animated fractal pattern that dynamically changes based on the output of an RNG, and they are asked to try and mentally influence the change of this pattern in a certain progressive direction (either making the pattern grow or shrink), mediated partly through a freely-determined volitional act on their part; namely, pressing the shift key on either side of a computer keyboard at times of their choosing. (In actuality, this latter aspect is only an illusory motivational cover: Although the participants are told that this volitional act is part of the influencing process, the button press itself merely serves to progressively activate the RNG sampling process for the next test trial, and has no other direct link to, or effect upon, the behavior of the RNG.) After participants have completed all of the test trials, the controlling computer automatically re-runs the full PK test again in a simulation mode (with no participant attempting to influence the pattern) to act as a matching control.

The analytical approach is then applied to the resulting data by constructing, for each test run and its subsequent matching control 
run, a correlation matrix which pairs the various physical variables collected in the course of that respective run with various psychological variables collected from the participants. The physical variables could include: the mean voltage output of the RNG, the standard deviation of that voltage, the number of times a " 1 " bit was generated by the RNG during a test run, and the number of times the changing fractal pattern had progressively deviated away from the intended goal direction. Psychological variables could include: the number of times the participant pressed the left shift key, the number of times the right shift key was pressed, the amount of time it took for the participant to complete the run, and various measures of personality characteristics. Based on the prediction, one would expect to find the test matrix to contain a higher number of significant correlations than would be expected by chance, and that there would be a significant difference between the test matrix and the control matrix in the number of significant correlations they contain. This is what has been found in only some of the small number of studies of this type that have been conducted so far: Supportive results (with z-scores $>2.00$ ) have been obtained in three of the five experiments conducted early on by von Lucadou (2006), and the most recent semi-independent attempt at replication (Walach et al., 2020) yielded a significant difference $(p=.017)$ between the test and control matrices. Other recent attempts at conceptual replication using a small number of physical and psychological variables (Grote, 2017, 2021) have found either only weakly suggestive $(p=.095)$ or chance results for the test matrix data, however, and additional replication data are needed for further clarification.

\section{Why Are Micro-PK Effects So Small?}

One commonality which seems to emerge from all of the experimental results examined so far is that although they tend to be quite significant, the PK effects observed in RNG studies also tend to be quite small in magnitude, even on the relative level of statistical outcomes. For instance, the two meta-analyses conducted by Radin and Nelson $(1989,2003)$ tend to indicate that the mean z-score for RNG-based PK experiments is only about 0.61 to 0.73 , and Helfrich (2007) pointed out that, although it is not given precisely, the mean $z$-score for the data reported in the Bösch et al. (2006a) meta-analysis 
can be estimated to be about o.6. In addition, the mean z-score obtained across the entire formal experimental database for the Global Consciousness Project is only about 0.33 (Nelson, 2015). Such scores suggest that the PK effects themselves tend to reflect only narrow fractions of a meanshift on average, and this may immediately lead some to wonder: Why do these effects tend to be so small, and not more relatively apparent?

While a clear and definitive answer has not yet been reached on this matter, one consideration recently put forth by Varvoglis and Bancel (2016) is that rather being an ability which is widely distributed across the entire human population, PK might instead be a more narrowly distributed ability which may be most frequently found among certain individuals who happen to be exceptionally adept at it (whether innately, or through developed practice). This suggestion was inspired by a closer re-examination of the PEAR benchmark RNG database, in which Varvoglis and Bancel (2016) had noticed that among the 91 individuals who participated in that experimental series, two of them particularly stood out from among the rest in terms of their exceptional PK scores: Whereas these two particular individuals had each achieved $z$-scores of 5.60 and 3.42, the other 89 participants in the database had collectively produced a z-score of only 0.82 . A similar observation was made in a review of the PEAR database by Dobyns (2015), who noted that: "An analysis of the distribution of effects among operators [i.e., participants] in the REG [experiments] finds that the best-fit explanation is a 'talented subpopulation' model in which 15 percent of operators are responsible for the anomaly" (p. 233).

One can also find similar distinctions elsewhere in the experimental PK literature, as well: In their meta-analysis, Bösch et al. (2006a) found that studies with individuals who were especially selected for participation (based on their success in prior PK tests, or on their claims of possessing psychic ability) had produced a significantly higher ( $p$ $\left.<1.00 \times 10^{-10}\right)$ overall $z$-score (6.87) than studies with unselected participants $(z=1.84)$. In evaluating the earlier PK studies with dice, Radin and Ferrari (1991) also found that participants selected on the basis of prior testing tended to produce higher effect sizes than unselected participants.

A potential caveat with these findings is that the total number of 
PK test trials collected so far with selected participants is much smaller than that collected with unselected participants, which leaves open the possibility that the exceptional scores obtained by the selected participants could be somewhat inflated. However, if additional data continue to indicate that scores obtained with selected participants remain considerably higher, then it may suggest that one way to possibly increase the size of PK effects on RNGs would be to focus study more toward these kinds of participants. Such a focus along these lines may also go some way toward addressing some skeptical arguments that micro-PK effects are too small to be worthy of much serious attention (e.g., Wilson \& Shadish, 2006).

\section{Addressing the Skeptics}

Despite the overall significance of their results, some professional skeptics have argued that there are a number of other potential issues with RNG experiments which do not allow them to be considered as evidence for micro-PK.

Several of these criticisms were leveled at $\mathrm{H}$. Schmidt's early research: For instance, Hansel (1981) generally argued that H. Schmidt's (1970a) significant results could have been produced through fraud, in three possible ways: First, in being isolated in the monitoring room during the test session, the experimenter could have tampered either with the RNG device or with the subsequently printed data record of its output. Second, in being left alone in the testing room with the visual feedback display, the participant could have tampered with the functioning of the test system by creating electrical shorts in the feed wires connecting the display to the RNG in the adjacent monitoring room. And third, any other person with knowledge of electronics could have similarly affected the test system's functioning by simply tapping into the same wire feed. However, with regard to such claims, $\mathrm{H}$. Schmidt (1987b) pointed out:

[Participant] fraud would have required, apart from specific electronics knowledge, much undisturbed time for opening the bottom plate of the testing machine and feeding in electric pulses in order to fool the internal counters as well as the external recorder. ... I was personally present in all tests, with the exception of a 
small part of the sessions with one [participant], and ... the scores in these few sessions were not higher than the other scores. In none of my subsequently reported experiments was there any less stringent [participant] supervision. (H. Schmidt, 1987b, p. 609)

Hansel (1981), Hyman (1981), and Alcock (1987, 1988) argued that there might have been randomization issues with Schmidt's work, in that he may not have conducted sufficient checks to ensure that his RNGs were producing adequately random output and were free of biased outcomes over the course of both short- and long-term sampling runs. However, H. Schmidt (1970a, 1973) did report several longterm randomness checks that were run outside of the experimental context (with no one being present), all of which were statistically nonsignificant and thus sufficiently in line with expected randomness. He further noted that none of these checks revealed any indications of biased output (H. Schmidt, 1987b), and Akers (1987), who utilized RNGs designed by Schmidt in his own experiments, had also stated:

I do not see any crippling design defects, such as generator bias, that provide an easy explanation for his results. Schmidt's control runs, though not strictly counterbalanced with experimental runs, were extensive, and they were conducted throughout the experiment. These control runs, even when cut into small segments, did not exhibit any evidence of short-term bias. (Akers, 1987, p. 567, emphasis in original)

Hansel (1980), Hyman (1981), and Alcock (1987, 1988) further claimed that rather than maintaining some degree of consistency by focusing on one particular type of RNG and/or test design, Schmidt would frequently skip from one type of RNG and/or test design to another, which kept him from "... focusing on a given research question, or refining his measurements, or examining the effects of various parameters in that particular situation, or working with one type of generator over a period of time so that he and others can come to appreciate its idiosyncrasies" (Alcock, 1987, p. 560). In response, $\mathrm{H}$. Schmidt (1987b) noted that although their circuitry components tended to change over time (following advances in electronics technology), all of his RNGs commonly utilized the same source of randomness: 
radioactive decay (or later, electronic noise; see H. Schmidt, 1973). $\mathrm{H}$. Schmidt (1987b) rationalized his changes in test design by further pointing out:

Even in a research effort that is very well focused, there will be side roads. We will want to explore, for example, other random generators and other forms of feedback to be reasonably sure that we don't overlook other, possibly more efficient, approaches to psi testing. On the other hand, we have to be selective because each study takes much time and effort. It therefore often seems more reasonable to postpone the study of some details until we have pursued the main questions that should contribute most to our understanding of the overall picture. (H. Schmidt, 1987b, p. 609)

And of the three critics, Hyman (1981) seemed to be the only one to partly recognize that finding similar PK effects occurring across multiple types of RNGs would go toward "... the desirable property of achieving generality among devices" (p. 37), helping to address the potential criticism that successful PK results obtained with one type of RNG might simply be due to bias-prone operational defects (or "bugs") in that particular type of RNG.

Alcock (1987, 1988) and Akers (1987) alluded to one RNG-PK experiment conducted by Schmidt that was particularly well-designed to guard against fraud and error, in that certain crucial parts of the procedure (namely, the random assignment of the target directions that participants should aim for, and evaluation of the resulting data) were supervised by independent observers (Schmidt et al., 1986). Although the overall result was significant $(z=2.71, p=.0032)$, Alcock and Akers both took a cautious "let's wait and see" stance, urging that further replications using the same type of design were necessary. It turns out that this particular experiment was the first in a series of five (Schmidt \& Braud, 1993; Schmidt et al., 1986; Schmidt et al., 1994; Schmidt \& Schlitz, 1989; Schmidt \& Stapp, 1993) that Schmidt conducted with independent observers. Three of these five experiments had overall outcomes at or exceeding the conventional level of statistical significance (i.e., $z \geq 1.64$, $p \leq .05$ ), and when evaluated altogether their results remained highly significant $(Z=3.67, p=.00012)$, with an associated odds ratio of about 8,200 to one ( $\mathrm{H}$. Schmidt, 1993a). This seemed to indicate that positive 
PK results were still achievable in Schmidt's experiments even when the conditions were tightly monitored and controlled.

Criticism was also leveled by Alcock (1988) at the PEAR research: Although he acknowledged the greater sophistication of the PEAR benchmark RNGs, Alcock (1988) still expressed concern about their randomness, asking: "[D]oes the machine when unaffected by the attempted influence of the [participant] produce output consistent with theoretical expectation" (p. 43)? To find out, the PEAR staff conducted extensive calibrations of their benchmark RNGs over the course of their 12-year program (Jahn et al., 1997), which, as noted previously, amounted to just over 5.8 million trials. The outcomes of these trials were found to conform well with the expected parameters of the underlying binomial distribution and were sufficiently in line with chance expectation overall $(Z=-0.83, p=.409)$. As an illustrative case in point, an examination of one subset of the calibration data collected by the PEAR staff, amounting to 50,000 calibration trials, reveals a flat, level random walk (Figure 8), with no consistent directional shift away from expectation being exhibited overall (Nelson et al., 1989).

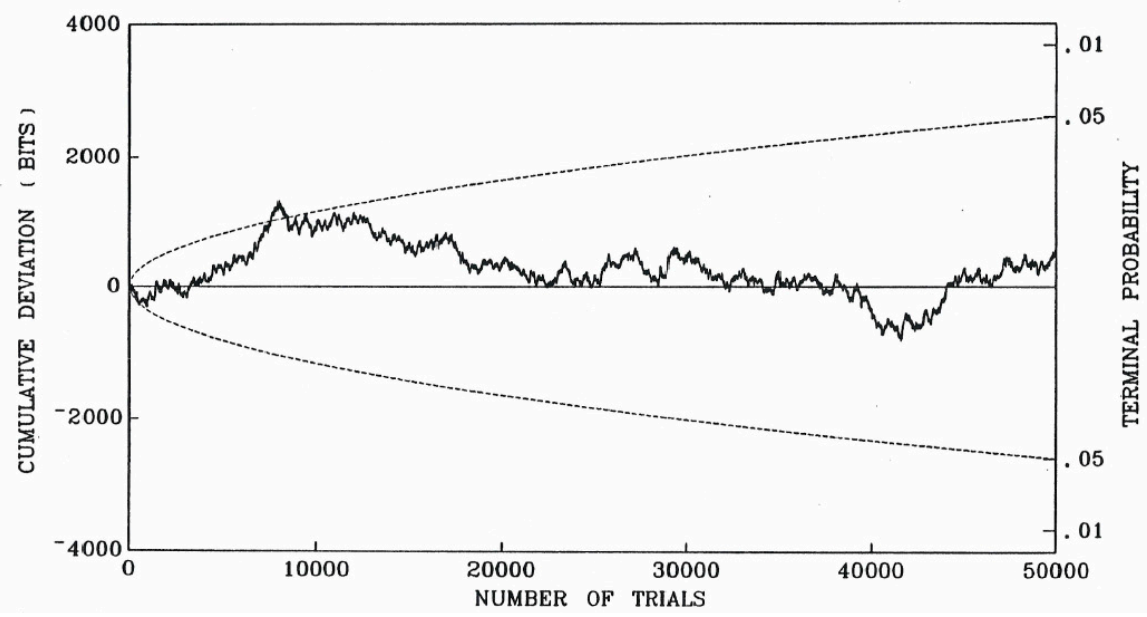

Figure 8. Cumulative deviation plot displaying an example subset of the extensive series of benchmark RNG calibration data collected by PEAR during its 12year experimental program. Compare the random walk exhibited by these calibration data with the illustrative example shown in Figure 2. Adapted from figure 7 of Nelson et al. (1989). 
Other criticisms of the PEAR research were later raised by Jeffers (1999, 2006), partly in relation to three micro-PK experiments which he independently conducted in collaboration with others (Freedman et al., 2003; Ibison \& Jeffers, 1998; Jeffers \& Sloan, 1992), and which were apparently motivated by the PEAR benchmark RNG program. Two of these experiments (Ibison \& Jeffers, 1998; Jeffers \& Sloan, 1992) had produced results consistent with chance, and have been touted in some skeptical circles as evidence which casts serious doubt on the results of the PEAR program. However, it is important to note that these two experiments cannot really be considered close replications of the PEAR benchmark RNG experiment, for they differed markedly from the latter in the type of PK target that was utilized: Rather than having participants try and mentally influence a benchmark-type RNG in these two experiments, Jeffers and his colleagues instead had them attempt to influence a more complex target: namely, the diffraction pattern produced by photons passing through a single- or double-slit screen (a well-recognized experimental setup often used to demonstrate the concept of wave-particle duality in physics). Although PEAR did conduct a few PK experiments early on using a similar kind of diffraction setup (Jahn, 1982, pp. 141-143; Nelson, Dunne, \& Jahn, 1982), they were not as extensive or as successful as the benchmark RNG program.

The third experiment (Freedman et al., 2003) came much closer to replicating the PEAR benchmark RNG test, in that an RNG similar in design to the benchmark-type was actually utilized as a PK target. But even that experiment might only be considered a conceptual replication at best, because as Dobyns (2003) pointed out, there were still several other differences of note: For instance, Freedman et al.'s participant pool comprised neurological patients and hospital staff, whereas the PEAR participant pool was made up entirely of healthy, unselected volunteers. Also, the only subset of Freedman et al.'s data that could be considered the closest in comparison with that of the PEAR database was the subset collected with the hospital staff (being the individuals who were healthy), which had a nonsignificant overall outcome. However, the number of PK test trials that Freedman et al. collected with these participants was much smaller $(94,000)$ compared to that of the PEAR database (over 1.6 million), which, considering the small size of micro-PK effects on average, might not have afforded enough 
statistical power to reliably detect such effects with these participants. (More will be said about this third experiment in a later subsection on neuropsychological factors.)

In discussing these three experiments in relation to the PEAR research, Jeffers (2006) has stated that:

One characteristic of the methodology in experiments in which I have been involved is that for every experiment conducted in which a human has consciously tried to bias the outcome, another experiment has been conducted immediately following the first when the human participant is instructed to ignore the apparatus [i.e., the slit set-up in the case of his first two experiments, or the RNG in the case of his third experiment with the neurological patients]. Our criterion for significance is thus derived by comparing the two sets of experiments. This is not the methodology of the PEAR group, which chooses to only occasionally run a calibration test of the degree of randomness of their apparatus. (Jeffers, 2006, pp. 55-56)

Although Jeffers (2006) has claimed that this method of control is "scientifically more sound" than the one utilized by PEAR (p. 56), Dobyns (2003) has pointed out several statistical and procedural ways in which it can potentially be vulnerable to artifacts and loss of statistical power. In addition, it can be argued that Jeffers' method may be susceptible to a potential confound; to see how, it is imperative to briefly draw attention to a few relevant points: The first is that knowledge regarding the duration and reach of PK (and psi in general) remains appreciably small at this point in time, and for that reason there is no reliable way known at present in which to strictly gauge or control the manifestation of PK within a given temporal period (in other words, there is currently no known reliable way, as of yet, to precisely begin or stop PK from occurring at a given time). The other point is that it seems that participants in micro-PK experiments do not necessarily have to direct their attention towards an RNG in order to be able to affect its output; perhaps one of the clearest lines of evidence suggesting this is that of the experimental findings obtained with field RNGs, where significant deviations were observed in the RNG data despite there being no overt signs of intention or attention being directed toward the RNGs by the involved groups. 
Arguably, both of these points could potentially factor into a test scenario akin to the one used by Jeffers (where collection of the control data immediately follows the collection of the experimental PK test data in a consecutive fashion) by considering the possibility that residual PK effects from the experimental period might sometimes unintentionally carry (or "bleed") over into the subsequent control period. Such a possibility has previously been given some consideration by the PEAR staff in relation to their field RNG experiments:

\begin{abstract}
When it is feasible to take [matching control] data in a given environment before and after the designated experimental segments, some of the surround time periods themselves may be subject to the same influences as the active segments. (Indeed, even in laboratory experiments there is evidence that traditional "control" data may not be immune to anomalous effects of consciousness). (Nelson et al., 1998, p. 452)
\end{abstract}

If this is plausible to some degree, then it may suggest that control data collected immediately following the experimental PK test could potentially be confounded as to whether the data would be totally free from the lingering effects of PK from the preceding experimental period, thus raising the question of whether they would constitute "pure" control data. On such a basis, one might argue that it would be preferable to temporally space apart the experimental and control periods in order to minimize any potential PK "contamination" of the latter.

Jeffers $(1999,2006)$ has further claimed that a problem exists with the baseline data collected by PEAR as part of the benchmark RNG program (the "BL" trace in Figure 3). He specifically points out that rather than maintaining a level random walk around expectation, the cumulative deviation plot of the BL data exhibits a modestly increasing shift away from expectation over the course of the program, which eventually exceeds the $p=.05$ threshold of statistical significance to a slight degree (with a terminal $p$-value of .04). Based on this observation, Jeffers (1999) argued "... that the behavior of the REG when examined over a 12-year baseline shows evidence of a slow but imperceptible drift indicating that it is not a random device as claimed [by PEAR]. Doubt must then be cast on the claimed terminal probabilities for the 
cumulative deviation plots with expressed operator intention [i.e., the HI \& LO data plots in Figure 3]" (pp. 328-329). He continued to maintain this argument several years later (Jeffers, 2006, p. 56).

However, it is important to carefully recognize that Jeffers' argument seems to hinge upon an assumption that the term baseline is synonymous with the term control in this case (an assumption that also seems to have been made by Alcock [1988, p. 39] in his own evaluation of the PEAR research). An implicit hint of this assumption is indicated in certain statements Jeffers has made in reference to the baseline data, where he framed them in the context of data being collected in situations in which participants ". . . are instructed not to interact with the [RNG] device" (Jeffers, 1999, p. 329, emphasis added) and “. . . in which no effort is made to bias the equipment" (Jeffers, 2006, p. 56, emphasis added).

Such a characterization of the baseline data as control data turns out to be inaccurate in this case, as a careful reading of PEAR's main experimental report on the benchmark RNG program (Jahn et al., 1997), as well as various other PEAR publications (e.g., Jahn \& Dunne, 1986, 2005; Nelson et al., 1986) would indicate that the baseline condition in fact represents one of the experimental conditions, where participants are indeed instructed to intentionally interact with the RNG, but only with the intent of trying to maintain as close to a steady baseline as possible. For this reason, the PEAR staff (Jahn et al., 1999) has cautioned that, in the case of their PK experiments with RNGs and other random systems,

... baselines never should be regarded as control or calibration data. They comprise a third condition of operator [i.e., participant] intention (albeit probably subconscious), and display many of the same structural features as the high- and low-intention data, including mean shifts.... Rather the proper comparison standards for the active [i.e., experimental] data, for the baseline data, and for qualification of the equipment, are the calibration data, taken with no operators (or anyone else) in the experimental room. (p. 330, italics in original)

As mentioned with regard to Alcock's criticisms, the calibration data were found to conform well to chance as expected (Figure 8).

Moreover, with regard to the baseline data, the PEAR staff(Jahn et 
al., 1997, 1999) pointed out that since there is no pre-specified prediction of an intentional shift in a certain direction (positive or negative) being made for those particular data, they should be properly evaluated using two-tailed statistics, leading the marginally significant terminal $p$-value of .04 for the overall outcome of the BL data (Figure 3) to be reduced to a nonsignificant .09. Thus, even if one were to take the baseline data as being control data, they still would not achieve a sufficient level of statistical significance for them to be viewed as being questionably non-random.

Among the more general claims that critics tend to maintain about experiments relating to micro-PK (and psi in general) is that there is a considerable (negative) correlation between experimental quality and effect size, such that PK effects start to decrease as more safeguards are added to the experiments. Meta-analytical assessments do not provide a clear indication of this, however, as only one RNG meta-analysis (Bösch et al., 2006a) has found a very small $(r=0.15, p=.004)$ correlation in line with this claim so far, ${ }^{9}$ while at least three others (Radin, 2006, p. 158; Radin \& Nelson, 1989, 2003) have uncovered no significant correlation. In addition, the odd standing meta-analysis (Bösch et al., 2006a) had found that the average quality of the experiments actually tended to be fairly high, with more than $40 \%$ of them being rated highly for implementing the safeguards being assessed (pp. 507-508). Thus, there may be some initial suggestion that this critical claim may not be a serious one, although further meta-analytical assessments would be necessary for better clarification.

Another general claim that some critics have maintained more recently is that rather than being reflective of a micro-PK effect, the small but significantly positive results obtained in RNG-PK metaanalyses may instead reflect the effect of selective reporting (also known as the "file-drawer" effect), where it is claimed that researchers have a tendency to report only those experimental findings that are in line with the test hypothesis (especially if they are significantly so), while actively suppressing the public release of any other findings which are close to chance and/or tend to refute the test hypothesis (by supposedly keeping those findings locked away in the drawer of a file cabinet); such a bias-driven practice would have the effect of artificially skewing 
the combined data more toward a significantly positive outcome. This has been proposed as a way to possibly account for the range of widely varying effect sizes that have been found within the experimental micro-PK database; selective reporting would tend to leave wide gaps between the outcomes of one experiment to the next, effectively making them non-uniform (or unbalanced) in distribution (Bösch et al., 2006a, 2006b; Pallikari, 2015; Schub, 2006).

To address this claim, some analyses have demonstrated that the results remain significantly positive $(p=.00033)$ even after being adjusted using statistical "trim and fill" algorithms designed to take the effects of selective reporting into account (Radin, 2006, p. 157), and an informal survey made by Radin et al. (2006a) of researchers in the parapsychological community who were known to have conducted RNG-PK experiments in the past had led to an estimate of about only 59 experiments going unreported, which falls well short of Bösch et al.'s (2006a) file-drawer estimate of 1,544 experiments that would be necessary to nullify the significance of their meta-analysis. Radin et al. (2006a) also note that some of these unreported experiments were said to have had significant results, which, if true, would stand in direct contradiction to the claim inherent in selective reporting that researchers actively seek to suppress negative or null findings.

In addition, Helfrich (2007) and Varvoglis and Bancel (2015) pointed toward there being an excessive amount of highly significant experiments (with z-scores $\geq 2.60$ ) contained within both tails of the PKRNG z-score distribution. As Varvoglis and Bancel (2015) have argued, reasonably accounting for these experiments by selective reporting can be quite challenging, as the existence of an extremely large file drawer would be necessary.

Radin et al. (2006a, 2006b) have also pointed out that it is possible that the wide range of RNG-PK effect sizes may be due to other factors besides selective reporting. They noted that some critics (e.g., Bösch et al., 2006a; Schub, 2006) have made the assumption that in these experiments, PK “... 'operates' by uniformly influencing each generated random bit regardless of the number of bits used per sample, the rate at which bits are produced, or the psychological conditions of the task" (Radin et al., 2006b, p. 362), and they argued that: 
The problem with this assumption is that there is no valid reason to expect that [PK] should behave this way. Indeed, we are unaware of any sort of human performance that is unaffected by such parametric changes. For example, a factory worker who can identify defective parts with $100 \%$ accuracy as they roll by at one or two per second will do no better than chance if the conveyor belt is suddenly accelerated to 1,000 parts per second. And yet the bit rate in the various [RNG-PK] experiments range over not just three orders of magnitude, but six orders, and they involve significant physical differences in the underlying mechanisms for generating random bits.

Then there are the psychological considerations. Let's say we conduct two RNG experiments. The first involves 1,000 highly experienced meditators, each of whom is selected based on his or her performance on previous, similar RNG tasks. Each participant is asked by a cordial, enthusiastic investigator to engage in a daily intention-focusing practice for six months in preparation for the experiment. When the experiment occurs, the participant is asked to intentionally influence the generation of a single truly random bit. The outcome of that one random decision will be provided as feedback either as a moment of silence (counted as a miss) or as a breathtaking fireworks display (a hit). If the fireworks display is observed, then the participant will also win something especially meaningful, like a scholarship or other accolade. In the second RNG study, a bored student investigator indifferently recruits an apathetic college sophomore, who is asked to press a button and mentally influence 1,000 random bits generated in one second, with no feedback of the results, and with no consequences regardless of the outcome.

The physical context of these two studies may be identical, employing the same RNG and the same statistics to evaluate the resulting datasets, each of which consists of 1,000 random bits. But it is clear that the psychological contexts differ radically. If we presumed that the only important factor in this type of experiment was the number of bits generated, then the two studies should provide about the same outcome. But if [PK] effects are moderated by variables such as the amount of time or effort one can apply in focusing mental intention towards the random events, or one's skill, or motivation, or how the bits were generated, then the first study might well result in an effect size orders of magnitude larger than the second. (Radin et al. 2006c, pp. 362-363)

Radin et al. (2006a, 2006b) further argued that a focus solely upon the physical aspects, while being appropriate for experiments devoted 
to pure physics, is not appropriate for experiments in PK, where psychological and other subjective factors can add a greater amount of variability that may ultimately affect the outcome.

As one means of addressing the issue of selective reporting in an empirical fashion, Bösch et al. (2006b) suggested that a psi study registry be established and broadly utilized, which would allow researchers to make the specific design and analytical details of any planned experiments (as well as meta-analyses) more widely available prior to proceeding with the collection of any data. Their suggestion has since become a realization under the auspices of the University of Edinburgh's Koestler Parapsychology Unit (Watt \& Kennedy, 2015, 2017), and should be considered for use in the development of future studies.

\section{PROCESS-ORIENTED RESEARCH: WHAT FACTORS MIGHT BE CONDUCIVE TO PK?}

If it can be assumed that the experimental results examined so far do provide some evidence for a micro-PK effect in totality, then the next question that might naturally come to mind is: Are there any physical, biological, psychological, or technical design factors that might affect micro-PK performance?

\section{Technical Design Factors}

Pseudo-random RNGs. While some types of RNGs can be considered "truly" random, in the sense that they have been designed to produce output based on purely physical processes which are inherently unpredictable, such as radioactivity (Aguayo et al., 1996; $\mathrm{H}$. Schmidt, 1970b, 1970c, 1977; Vincent, 1970) and electronic noise (Nelson et al., 1989), there are also some types of RNGs that can be considered "pseudo"-random, in which they have been designed to base their outputs on processes which are inherently nonphysical and more deterministic, such as mathematical algorithms (for a general overview of these latter types of RNGs and their possible limitations, see Radin, 1985). One might be led to wonder: If human mental intention can potentially influence the output of truly random RNGs, then could it also potentially influence the output of pseudo-random RNGs, as well? 
One of the earliest efforts to explore this question was made by Tremmel and Honorton (1980), who examined the performance of more than 40 unselected participants on two PK tests using electronic noise RNGs, as well as on a third test using an algorithm-based RNG. While the combined data from the tests with the noise-based RNGs resulted in significantly above chance scoring $(z=2.47, p=.018)$, the test data with the algorithm-based RNG were found to be nonsignificant.

In one of his experiments, $\mathrm{H}$. Schmidt (1981) used binary number sequences pseudo-randomly derived from an algorithm to control the directional rotation (clockwise or counterclockwise) of his lamp-lit circular visual feedback display. Significant results were obtained with both unselected volunteers $(z=2.19, p=.014)$ and selected participants $(z=3.42, p=.0003)$. One should note, however, that the test sequences used in this experiment were not pseudo-random in the purest sense, in that the starting seed numbers (which were entered into the algorithm to generate the sequences) were initially obtained beforehand in a truly random fashion through radioactive sampling.

Lowry (1981) designed and participated in two computercontrolled experiments in which he attempted mental influence upon a series of random digits (ranging from 1 to 4 ) being generated from an algorithm activated 0.2 seconds after the press of a key at the start of each test trial. The first experiment resulted in a success rate that was significantly below MCE (24.1\%, where $25 \%$ was expected; $z=-2.61, p$ $=.009$, two-tailed), although a cluster of positive scores was found in the first half of the test runs. Efforts to reproduce this positive cluster became the focus of the second experiment, which resulted in a success rate significantly above $\operatorname{MCE}(27.1 \%, z=4.23, p=.000023$, two-tailed).

In his effort to replicate Schmidt's work, Radin (1982a; Radin \& Utts, 1989) conducted a series of 27 experiments in which he and other selected or unselected participants each attempted to influence an RNG controlling the lamp-lit circular visual feedback display. The RNG could be set up so that its output was produced either in a truly random fashion (based on radioactive sampling) or in a pseudo-random fashion (using seed numbers fed into an algorithm). The combined data across the entire experimental series resulted in a significant outcome $(Z$ = 2.94, $p=.002$ ), and while not being significant on their own, the experiments in which pseudo-random number sequences were used 
had modestly contributed to the positive outcome $(Z=1.12)$.

Radin (1982b) further carried out a series of four experiments using computer-based tests relying on pseudo-random number sequences derived from the 32-bit UNIX Rand algorithm function. Overall, five of the 18 statistical tests performed on the resulting data from these experiments were found to be significant at the $p<.05$ level (exact binomial $p<.005)$.

Initially, most of these findings seem to stand in contrast with those of PEAR (Dobyns, 2015, pp. 225-227; Jahn et al., 1997; Nelson et al., 2000), which, in an extensive series of just over 400 experiments, found no significant indications that algorithm-generated pseudorandom number sequences (seeded by stops of a computer clock) could be influenced by mental intention. However, closer examination has seemingly revealed possible hints of modestly deviating $\mathrm{HI}$ and LO trends present in the PEAR pseudo-random data (with one acting in line with, and one acting opposite to, intention), which may be counteracting each other in such a way that happens to lead to an overall null result (Jahn \& Dunne, 2011, Ch. 6).

Thus, while a small number of experiments seem to initially suggest that pseudo-RNGs can possibly be influenced by PK, some degree of ambiguity still surrounds the issue and further clarifying data are needed.

RNG Bit Rate. Does increasing the number of bits being sampled from the RNG per second affect micro-PK performance in any way? As mentioned previously, initial exploration of this was made early on by H. Schmidt (1973), when he compared the scores obtained in PK tests where the RNG was set to run at two different bit generation speeds: a relatively slow speed ( 30 bits per second), and a relatively fast speed (300 bits per second). Though quite small, a significant difference $\left(z_{\text {diff }}\right.$ $\left.=4.75, p=1.02 \times 10^{-6}\right)$ was indeed found between the slow $(51.6 \%)$ and fast $(50.3 \%)$ success rates, on average.

H. Schmidt (1974b) further explored the question in a more indirect fashion through a formal study in which he compared the scores obtained in PK tests involving a relatively simple RNG (where a single bit was generated for each test trial based on radioactive sampling) with those obtained in tests involving a relatively complex RNG (where the bit for each trial was instead determined in a more complex fashion 
by taking a majority count-either of "1"s or "O"s—of a sequence of 100 binary numbers rapidly generated by sampling electronic noise). While a pilot experiment did initially indicate a significant difference $\left(z_{\text {diff }}=2.20, p=.028\right.$, two-tailed) between the simple (58\%) and complex $(51.2 \%)$ success rates, it was not found again in a subsequent and larger confirmatory experiment, although the average success rate with the simple RNG remained slightly higher (55.3\%) than that with the complex RNG (53.8\%).

Exploration of this question was also independently pursued by the staff of PEAR (Dobyns et al., 2004; Jahn et al., 1997) several years later, when they began looking at how the PK effect sizes changed as the bit rate on the benchmark RNG was set at three different sampling speeds: 20 bits per second, 200 bits per second (the standard rate used in most PEAR experiments), and 2,000 bits per second. The particular aim here was to explore a "bitwise influence" model of PK, which posits a possible PK operation in which mental intention is assumed to influence each individual bit being generated by the RNG, shifting its underlying probability more toward the likelihood of an intended (or desired) outcome. If PK happens to operate by such a mechanism, then one might predict that as the number of generated bits is increased, PK effect sizes should also start to increase, as well (in other words, higher bit rates should lead to bigger PK effects, according to this model).

Initially, the resulting data seemed to indicate that the PK effect sizes were indeed gradually increasing in a semi-linear fashion as the bit rates got higher; in other words, the PK effects did seem to be getting slightly bigger as the number of sampled bits went up, just as the bitwise influence model would predict. However, further study by Ibison (1998) also seemed to indicate that when the rate was set extremely fast (using a high-speed electronic noise source that generated 2,000,000 bits per second), a marked change unexpectedly occurred: The PK effect inverted itself from positive to negative, to a highly significant degree $(Z=-3.56, p=.00037)$. On the surface, such a finding would seem to contradict the bitwise influence model.

This latter finding spurred a second, replication-oriented phase of this experiment (dubbed "MegaREG"; Dobyns et al., 2004) by PEAR. PK test trials generated at a rate of 2 million bits per second were randomly interspersed with trials of 200 bits per second, with both participants 
and experimenters being kept unaware of which trials were which (so as to control for any differences in performance or analysis that might result from having distinct knowledge of the two conditions); the remaining features and procedures of the PK test were otherwise kept the same as those used in the benchmark RNG experiment. A highly significant result was found for the 2 million-bit rate trials that was again flipped in sign $(T=-4.03,105 \mathrm{df}, p=.000057$, two-tailed $),{ }^{10}$ again contradicting the prediction of the bitwise influence model.

However, the initial rise in PK effect size that was observed as the bit rate increased from $20 \mathrm{bits} / \mathrm{sec}$ to $2,000 \mathrm{bits} / \mathrm{sec}$, followed by the sudden fall from positive to negative at 2 million bits/sec, would seem to hint at the possibility that perhaps somewhere between 2,000 bits/ $\mathrm{sec}$ and 2 million bits/sec, there might be a particular "optimum" bit rate at which the PK effect size attains its highest value (i.e., it "peaks"), and beyond which the effect size inverts direction and starts to decrease. Could there be such a hypothetical "optimum" bit rate? Exploration of this question was pursued by K. Alexander (2019) as he sought to independently replicate and expand upon the MegaREG experiment. In his study, participants were asked to try and influence the output of a custom-made, four-unit RNG device that could be set to run at any one of 10 differing bit rates ranging from 200 bits/sec up to 16 million bits/sec. Although there was an indication that the PK effect sizes were gradually increasing again as the bit rates got higher (as suggested by another positive semi-linear trend, which was in line with the bitwise model prediction and inconsistent with the inversion observed in the original MegaREG experiment), the resulting data remained somewhat ambiguous because the effect sizes also seemed to be alternately switching from positive to negative (and vice-versa) in a "zigzagging" fashion as the bit rates got higher (i.e., some bit rates were correlated with a positive effect size, while others were correlated with a negative effect size). Thus, it still remains unclear as to whether PK performance might be affected by bit rate, and additional clarifying data are needed.

\section{Physical Factors}

Spatial Distance. Several of the known physical forces, such as gravity and the electrostatic force, have been found to obey a limitation that depends on spatial distance, which can be mathematically modeled 
and described by the inverse-square function: The strength of the force decreases as the inverse square of the distance from its source (Young, 2012, Sect. 0.4). If one were to assume, simply for the sake of conceptualization, that it might represent a unique kind of force (namely, a mental "force" with some degree of direct physical analog), then the question arises: Might PK have any kind of spatial limit of its own?

One aspect of the PEAR program (dubbed "Remote REG") had initially explored this question over a six-year period by having participants attempt to influence the output of a benchmark RNG from various distances away, with the spatial separation between the participant and the RNG ranging from less than one mile to more than 5,000 miles (Dunne \& Jahn, 1992). Nearly 1.5 million bits were generated in total, and like with the standard (local) benchmark RNG experiment the overall result (again expressed as the difference between the $\mathrm{HI}$ and LO conditions) was small but significant $\left(Z_{\text {diff }}=2.23, p=.013\right)$, suggesting that the participants' attempted PK influence was effective regardless of how far away they were from the RNG. The results suggested that the influence tended to be most effective in the $\mathrm{HI}$ condition, which was highly significant $(z=3.19, p=.0007)$.

The spatial question was further explored in two experiments conducted by Tressoldi et al. (2014) just over two decades later. In the pilot experiment, small groups of three to seven participants were asked to try and collectively influence the output of a remotely activated RNG located 190 kilometers (approx. 118.1 miles) away over the course of a relatively short influence period (ranging from 1 to 3.3 minutes). Success was defined by the RNG's output achieving or exceeding a defined cutoff threshold set at the conventional level of statistical significance (i.e., $Z \geq 1.64, p \leq .05$ ) in either direction (positive or negative). Out of the 50 pilot sessions, 39 of them (78\%) were found to have reached or exceeded this threshold. In contrast, only about half (48\%) of the matching 50 control sessions had reached or exceeded the threshold, with the difference favoring the PK hypothesis by a Bayesian odds factor of 52.4 .

The larger confirmatory experiment comprised 102 test sessions in which participants attempted to individually influence the remote RNG's output over the course of a shorter influence period (one minute). 
Spatial distance between the participant and the RNG ranged from 4 to 1,512 kilometers (approx. 2.49 to 939.5 miles). Eighty-four (82.3\%) of the test sessions were found to have reached or exceeded the threshold, whereas only $14(13.7 \%)$ of the control sessions did so-a difference again favoring the PK hypothesis by a Bayesian odds factor of $7.3 \times 10^{11}$.

Tressoldi et al. (2020) later conducted a third experiment to explore the spatial question in which participants individually tried to influence a custom-built RNG device located between 15 and 4,000 kilometers (approx. 9.3 to 2,485 miles) away, over the course of a 15-minute Skypebased test session (again with a one-minute influence period for each of the 15 test trials that comprised the session). Compared to the matching control data, the experimental data were found to exhibit an approximately $50 \%$ higher amount of trials which met or exceeded the success threshold (again set to $Z \geq 1.64, p \leq .05$ ).

An examination of the spatial question was also made by Bancel (2015) in a field RNG context using the data gathered by the Global Consciousness Project, in order to see whether the RNG network data associated with major world events (i.e., those which tend to have a widespread impact upon human populations spread around the world) might be notably distinct from the data associated with relatively minor world events (i.e., those with an impact which may be more limited to relatively smaller populations located in certain regions of the world). And indeed, a significant difference $\left(Z_{\text {diff }}=2.20, p=\right.$ .014) was indicated: The presumed group PK effects associated with minor world events seemed to exhibit a significant decline $(Z=-2.56)$ with increasing distance from the area where the minor events were taking place, suggesting that the effects tended to be limited to the RNG network nodes positioned in the geographic areas immediately surrounding those events. This contrasted with the effects associated with major world events, which did not exhibit any such decline ( $Z$ $=0.76$ ), suggesting that these effects tended to be distributed more globally across the various nodes of the RNG network.

These findings would seem to initially suggest that micro-PK does not really exhibit much of a spatial dependence, although additional replications would be valuable in further confirming this. It is also important to note that the PK influence periods used in the experiments by Tressoldi et al. (2014, 2020) were relatively short in length, and given 
the small effect sizes that tend to be associated with RNG-based micro$\mathrm{PK}$, longer periods of sampling may be a crucial factor to consider in any future replications.

Geomagnetic Correlates. Considerable effort has been made over the years to determine whether fluctuations in the Earth's magnetic field, which can often be influenced by the solar wind (Campbell, 1997; Lyon, 2000), might have an effect upon human health and behavior (Close, 2012; Palmer et al., 2006; Zenchenko \& Breus, 2021). This may lead one to wonder: Might psi phenomena possibly exhibit any correlation with geomagnetic activity? A number of attempts have been made to empirically explore this question with regard to ESP, and while there seems to be a hint of a possible inverse (or negative) correlation in some cases, the study designs and results have generally been mixed and the answer remains far from clear (Ryan \& Spottiswoode, 2015).

Fewer studies of this type have been done so far with regard to micro-PK: One of the earliest attempts was conducted by the PEAR staff in the late 1980 (Nelson \& Dunne, 1987) by statistically comparing their extensive benchmark RNG database with the average antipodal (aa) index (a daily measure of geomagnetic activity derived from data collected from two monitoring stations, with each positioned in one of the Earth's two hemispheres; Campbell, 1997, p. 167) for the northern hemisphere. A slight negative correlation was indicated $(r=-0.028)$ only for the RNG data in the "LO" condition, although it was not significant overall. No other indications of a correlation were found for the rest of the RNG database.

Three attempts were made in the 1990s: In the first, Gissurarson (1992b) compared the scoring data from eleven PK experiments using an RNG-based computer game design with the $K$-index values (a threehour average measure of horizontal geomagnetic field variation based on data collected from 11 or more monitoring stations positioned around the world; Campbell, 1997, p. 161) obtained from stations locally positioned closest to the laboratories where the experiments were conducted. No significant overall correlation was found, although there was a weakly suggestive hint $(p=.078)$ that "[h]igh scores were preceded by high geomagnetic activity, and low scores were preceded by low geomagnetic activity" (p. 163).

In the second, Bierman and van Gelderen (1994) compared the 
planetary $A$ index (PAI) values with the data from a computer-based PK experiment in which 16 participants tried (on two separate occasions) to influence an RNG programmed to generate bits at a fast and a slow rate. Only a slightly negative but nonsignificant correlation $(r=-0.19)$ was found overall.

In the third, Broughton and Higgins (1994) each tested themselves in a separate series of runs on a computer-based PK test, and their scores on the initial runs for each test in the series were combined and compared against the $A p$-index values (an averaged planetary measure derived from the $K$-index that reflects "the equivalent daily amplitude"; Campbell, 1997, p. 163). A slightly negative correlation (Spearman's rho $=-0.17$ ) resulted, which was also nonsignificant overall.

Most recently, Caswell et al. (2014) examined the Kp index values (the averaged planetary measure of the $K$-index values taken across 11 monitoring stations; Campbell, 1997, p. 161) in relation to PK test data collected from 26 participants who tried to influence a computercontrolled RNG. Significant Pearson $(r=0.54, p<.01)$ and Spearman ( $r h o=0.41, p<.05)$ correlations resulted when the data at the time of the test were compared with data taken three hours before and after the test, although some limitations are important to note with regard to these results: First, the participants in this study took part in only one test session, with the PK influence period being only about five to eight minutes in length, which (again considering the small effect sizes involved in RNG-based PK tests) is likely to be too short of a sampling period to reliably detect any micro-PK effect. Second, the $K p$ values were compared against RNG-PK test data averages of only one minute in length, and in using such short temporal samples, it is not clear how deviations produced by simple chance-expected fluctuations in the random background noise of the RNG were taken into account. In addition to making it unclear whether they might be due to PK (as there is currently no unambiguous way to distinguish between small PK-related deviations and fluctuations of pure noise at this narrow level of examination), such short-lived fluctuations in the RNG data could have potentially inflated the resulting correlations.

Although some of these findings seem to hint at a possible negative correlation between RNG-PK and geomagnetic activity, the relative lack of statistical significance across studies tend to make this 
far from clear; part of this issue may be related to the relatively small RNG sample sizes used. In addition, Hubbard and May (1987) have pointed out that since a geomagnetic index

\begin{abstract}
... is determined from a weighted average of data reported from [multiple] worldwide measurement stations, the GMF activity may be relatively quiet in one area while the index is reflecting a magnetic disturbance detected at several other sites. Geomagnetic indices are therefore poor indicators of the local magnetic conditions that may possibly influence psi performance. (Hubbard \& May, 1987, p. 80)
\end{abstract}

To further explore any possible correlation with geomagnetic activity, they suggest that psi test data should instead be examined in relation to direct local measurements of the ambient geomagnetic field taken in the immediate area where the psi test is being conducted.

Some exploratory findings by Stevens (2005) would also seem to hint at the initial possibility that ". . . the REG target system used in many micro-PK studies might be a sensitive detector of geomagnetic fluctuations, possibly through a process of stochastic resonance with intrinsic and/or external noise" (p. 143), which might account for some of the varying findings observed in PK studies using RNGs, though this remains a tentative consideration at present. ${ }^{11}$

Some findings by Radin (1993b, 1996) would also seem to hint at the initial possibility that PK (and perhaps psi functioning in general) could be correlated with more than one physical variable in a rather complex way, a consideration that may make the search for potential correlates on the individual level a bit more challenging.

\title{
Biological Factors
}

Gender. The question of whether there might be any distinct cognitive (and other behavioral) differences between females and males has been the subject of much study and debate (Miller \& Halpern, 2014). This may lead some to wonder: Do females and males show any differences in psi performance? Many of the study findings have presented a mixed and unclear picture with regard to ESP (Schmeidler, 1994, p. 152), but what about for micro-PK? To date, addressing the latter question has been quite limited: 
In his examination of the data from 11 PK experiments using an RNG-based computer game design, Gissurarson (1992b) found a significant directional difference $\left(F_{1,619}=5.25, p=.02\right.$, two-tailed) between the mean scores of male and female participants, with males tending to score slightly above chance, and females tending to score slightly below chance.

A more extensive examination was later conducted by Dunne (1998), using the PEAR benchmark RNG database. The combined results indicated that the 41 female participants contributing to the database had tended to achieve significantly positive scoring $(z=3.339, p=.0004)$ on the "HI" condition, whereas the 50 male participants achieved a more modest level of scoring $(z=1.228)$, leading to a higher overall $\mathrm{HI}-\mathrm{LO}$ difference $\left(Z_{\text {diff }}=3.382, p=.0004\right)$ for females as compared to males $\left(Z_{\text {diff }}=1.875, p=.03\right)$. Part of this difference might be attributable to the larger data contributions made by females, however, with the female average database being nearly twice as large as the male average database. Some of these differential effects associated with females also seem to extend to other parts of the broader PEAR program database, as well (see Ch. 13 of Jahn \& Dunne, 2011, for a concise overview).

\section{Mental Factors}

Co-Op Efforts. Might micro-PK effects start to linearly increase (or "get stronger") if two participants were to simultaneously focus their mental intention efforts on the same RNG? This question was initially explored by Dunne (1991) in a subset of 42 experiments within the PEAR benchmark database, in which pairs of selected participants attempted to influence the same RNG in a semi-cooperative manner by aiming their influence efforts in the same direction. Overall, this experimental subset resulted in a modestly significant $\mathrm{HI}-\mathrm{LO}$ difference $\left(Z_{\text {diff }}=\right.$ $1.883, p=.03$ ), with the size of the "co-op" effects being slightly (about 3.7 times) larger than those observed in efforts made by individual participants alone. Despite this, there were some indications that the efforts of paired participants did not simply increase in a linear fashion; as Dunne (1991) pointed out:

... some of our most successful individual operators [i.e., participants] produce null effects when working together, while others 
with minimal or even negative individual results are able to produce strong positive yields as a pair. (p. 4)

This observation seems somewhat consistent with those made in earlier "co-op" PK studies conducted using dice (Feather \& Rhine, 1969, 1971; Humphrey, 1947), which were also often mixed in outcome such that "... when the two [participants] tried for the same target face they either succeeded very well or missed very badly" (Feather \& Rhine, 1971, p. 91).

In addition (and further extending the data relating to gender), efforts made by pairs of participants of the same gender were nonsignificant and tended to be in the direction opposite of the one they were cooperatively aiming for. Yet in a curious fashion, efforts made by participant pairs who were of opposite gender tended to achieve results that were in the intended direction, and which were significant overall $(z=3.317, p<.0005)$.

The reasons for these mixed outcomes among "co-op" pairs remains unknown, and with so few studies having been done so far, further clarifying data are needed.

Gaming/Competition. On the opposite end of the spectrum to "co-op" efforts are instances in which participants attempt to aim their mental influence efforts in opposing directions, in a semi-competitive fashion. As one means of trying to motivate participants and (in some cases) encourage this kind of playful competition, some RNG-based PK tests were designed to have a visually engaging electronic game-type interface; this largely began in the 1970 s and 1980s, with the advent and wider availability of the personal computer (PC).

Two early tests of this type were used byWeiner $(1978,1979)$ : The first one was a PC game designed to conceptually simulate the conditions of a competitive horse race, with the "horses" being represented by four columns of numbers that would separately increase at random over the course of a 50-trial "race." Each of the columns would start at zero and begin counting upward as the race was under way, with the output of an RNG randomly determining the moments when each one would be incremented to a higher value (so that at the end of the race, each column could result in a different value). The playful engagement aspect of the test involved asking participants to choose one of the four "horses" (columns) to lay a wager on (with varying levels of monetary 
risk), and then having them try to mentally influence their horse's placing in the race (presumably through PK on the RNG) such that it would achieve the highest numerical value in the end (and thereby win the race). None of the main analyses performed on the resulting RNG data were significant.

The second one was a PC test designed to explore a possible relation between PK and motor skill by having participants attempt to influence the unsteady motion of a bar on a computer screen, such that it would remain centered on the screen for as long as possible and not move off the edges of the screen. In one part of the test, the participant attempted this manually by using a control dial to help keep the bar steady. In another part, the participant attempted this using PK, with an RNG randomly determining whether the behavior of the bar would become increasingly unstable or not during each test trial. Although male participants had attained higher PK scores and motor skill results than females, the difference was found to be only suggestive $\left(F_{1,24}=3.94, p=.056\right)$.

Broughton (1979) asked participants to interact with an electronic RNG-based PK test device that was designed to mimic the familiar carnival tests of physical strength, in which a person uses a mallet to hit a lever with as much strength as they can muster in an attempt to send a weight high enough along a wire to hit a bell at the very top (only with this device, participants attempted this with their minds instead of their muscles, making it a test of "psychic strength," in a sense). Participants were tested individually or in a small, playfully competitive group setting among friends, but no significant deviations from chance were observed overall.

Honorton and his colleagues at the Psychophysical Research Laboratories (PRL) developed three types of RNG-based PK games, two of which utilized the custom-designed PsiLab II hardware RNG and associated software running on the popular Apple II computer of the 1980 (Berger \& Honorton, 1985; Berger et al., 1986; PRL, 1985). One of them, called "Volition," produced a running display of the RNG output in a manner roughly equivalent to that of the cumulative deviation plot utilized by PEAR (Figure 3), showing the bit output for each test trial as it randomly fluctuates in direction. The other, called "Psi Invaders," was a modified adaptation of the popular 1980 s video arcade game "Space 
Invaders," in which players attempt to use a horizontally-sliding laser gun turret to shoot and destroy descending rows of invading enemy spaceships while they avoid being hit and destroyed themselves by the enemy's own array of bombarding laser fire (this gets increasingly more difficult as the game progresses, as the rows of invading spaceships gradually begin to move and descend at a faster pace). The PK aspect of the game was integrated into the operation of the laser gun affixed to the player's turret, which was said to be "old and frequently misfires." Whether or not the gun will fire when the player presses the gamepad button is randomly determined by the RNG output, and the player is asked to use PK in attempt to make it fire more often.

The third game, called "Psi Ball," was conceptually similar to Weiner's (1979) motor skill PC test, in that players moved a lever in attempt to keep a ball on the computer screen away from the "wall" at the edges of the screen. The RNG output determined whether the ball would become increasingly more sensitive to small movements of the lever (thereby increasing the difficulty of the game), and players would try to minimize this by using PK. These three games could be set to run in two different modes: "Feedback," in which an overt change in some aspect of the game's dynamics (e.g., its pace, its direction, or its screen color) could give players real-time visual feedback on the RNG's output (and thus, their PK performance); and "Silent," in which none of the game dynamics were overtly affected by the RNG output, and feedback was given to the player only after the game was over (or, in some cases, not given at all).

Experiments with these three PK games were conducted over the course of the 1980s by the PRL staff (Berger, 1988a, 1988b; Honorton et al., 1983; Schechter, Barker, \& Varvoglis, 1983, 1984; Schechter, Honorton, Barker, \& Varvoglis, 1984; Varvoglis, 1989) and by other researchers (Gissurarson, 1986; Palmer \& Perlstrom, 1987) with varied test outcomes ranging from null (e.g., Schechter et al., 1983, 1984; Varvoglis, 1989) to suggestive (e.g., Honorton et al., 1983) to significant (e.g., Berger, 1988b; Gissurarson, 1986). One notable finding which seemed to emerge from several of them was a tendency for some participants to produce positive PK scoring while the games were running in "Silent" mode (Berger, 1988b; Berger et al., 1986; Palmer \& Perlstrom, 1987; Varvoglis, 1989), offering initial suggestion that 
perhaps some participants may be able to score well in the absence of real-time feedback (although in some cases, the "Silent" RNG data were viewed only by the experimenters, and not the participants, which may lend some consideration toward an experimenter psi effect, an alternate possibility that will be raised in a later section).

Another type of PK game test, utilized by Broughton and Perlstrom $(1985,1986,1992)$, was a modified version of a commercially available computer-based dice game in which players took turns rolling a pair of electronic dice (randomly controlled by an RNG) on a screen and aiming for high scores while trying to avoid rolling doubles (which decreased the score). The test was first used in a short series of three experiments in which Perlstrom acted as the sole participant playing against the computer. The combined RNG data from all three experimental series resulted in a significant departure from chance $(Z=4.841, p<.0001)$, whereas data from a matching control series were consistent with chance (Broughton \& Perlstrom, 1985).

The test was again used in another series of three experiments later conducted with volunteer participants who took part in a (sham) competitive test scenario, in which they were led to believe that they would be playing the game against an opponent on another computer connected via a modem link-up (when in actuality, there was no other human opponent and they were just playing against an imaginary opponent being simulated by the computer). Since many of the participants were college students recruited from Duke University, they were further told that their opponent was another student from local collegiate sports rival University of North Carolina, Chapel Hill, so as to further encourage a competitive school spirit in them. Although the combined RNG data from all three experiments were at chance, the overall results did seem to be consistently indicative of there being an inverse correlation (amounting to a combined $Z=4.1, p=.00004$, twotailed) between the level of anxiety reportedly felt by the participants and their subsequent scores on the competitive PK test (Broughton \& Perlstrom, 1992, p. 302).

One other type of PK game test, utilized in experiments by Hansen (1990) and Roe et al. (2004), followed Weiner's (1978) earlier PC-based test in simulating races with animals, this time using digitally animated horses and greyhounds, respectively, moving across a computer screen. 
As in Weiner's test, RNG output was used to randomly determine the moments when a given race animal would take its next incremental step forward, and participants attempted to influence this via PK for their designated race animal. Hansen's (1990) experiment compared both a competitive condition (where participants separately attempted to influence their own individual horses) and a cooperative condition (where participants attempted to influence the same horse, only without being aware that they were doing so, making it subtly distinct from the mutual "co-op" experiments discussed previously), with the prediction that the latter condition would produce higher PK scores. The data resulted in the opposite outcome $\left(Z_{\text {diff }}=-2.054\right)$, however, for reasons that were unclear. In addition to comparing it against an ESP condition, the experiment by Roe et al. (2004) examined whether participants' performance in a PK test condition was affected by either a state of high arousal or a state of passive calmness. Overall results in the PK task were at chance.

Thus, while it seems so far that RNG-based games have generally not led to improved PK performance, and that competitive conditions have been mixed in outcome, the number of experiments again remains relatively small and certain preliminary correlations have emerged from these types of studies that may be worth further exploration using PK tests that can be integrated into the more widely accessible and advanced computer and video game console technology that is presently available.

Psychic Signatures. Although human bodies all tend to be biologically structured in a similar way on the large scale, there is one bodily structure existing on the fine scale which happens to be unique enough across individuals that it can often be used as subtle personal identifier (or "signature"): the whorls of the human fingerprint. Could there be any kind of unique "signature" to a person's psi performance that might allow it to be individually attributable to that particular person?

Some preliminary consideration of this question had arisen in relation to certain scoring patterns that were unexpectedly seen in the data collected over the course of a test series in some of the early PK studies with dice (McConnell, 1989). Appearing from time to time in the presence of a certain experimenter, these patterns seemed similar 
enough in their general structure across the tests that they raised the issue of a possible experimenter psi effect (to be discussed later).

Other suggestive scoring patterns that seemed to be associated with certain test participants also were observed in the PEAR benchmark data (Babu, 1987; Nelson et al., 1986, pp. 266-268). Of this, it was noted that:

Some operators [i.e., participants] achieve PK results in only one direction, some in neither, some in both, and some show inverted results. The $[\mathrm{HI}]$ and $[\mathrm{LO}]$ achievement patterns for a given operator are typically asymmetrical, and are often found to be dependent on the conditions under which the operator is performing the experiment, such as the pulse [i.e., bit] counting rate, whether each trial in the [test] run is initiated manually or automatically, or whether the operator chooses or is randomly assigned the direction of effort. (Nelson et al., 1986, p. 266)

Focused exploration of the question was initially undertaken in a pilot experiment by Berger (1988a), in which eight participants each completed two test series on Psychophysical Research Laboratories' "Volition" PK game. The resulting RNG data produced by each participant in their first series were then statistically compared with the data from their second series in order to see if the two sets of data might be notably similar in any way with regard to their scoring patterns (as would be inferred from the presence of a significantly positive correlation between them). Two of the eight comparisons resulted in a significant correlation, a suggestive outcome (binomial $p=.057$ ). As a further test, the data from each participant's first series were blindly compared against a randomized set of data from 21 other test series: One of the series contained in the set was the participant's actual RNG data from their second series, while the other 20 series were simulated RNG data produced by the computer to act as "decoys." A Pearson correlation coefficient was calculated for each statistical comparison, and the resulting 21 correlation values were then ranked from highest to lowest, with a 1 in 21 (or .048) probability of the participant's actual RNG data being ranked the highest. Overall, the participant's data was found to have been ranked highest in three out of the eight cases, resulting in a significant binomial probability of .005 . 
Further exploration was made in two studies conducted by Radin $(1989,1993 a)$. In the first study, an artificial neural network was used to see if it could possibly recognize and identify individual participants on the basis of any subtle but unique scoring patterns that might be present in their PK test data. To do this, data collected from 32 participants who had taken part in the PEAR benchmark RNG program were divided into two halves: The first half (consisting of RNG data individually produced by each of the PEAR participants in a PK test) was used to "train" the network by running the data through the network hundreds or thousands of times, first in a feed-forward fashion, followed by a back propagation of the data (i.e., running them back through the network in reverse); this method of computation allowed the network to initially "learn" to associate any patterns that might be present within the individual data with a particular participant based on the multiple unique interconnectionist (or "linked") information processing pathways that it forms between its nodes (roughly resembling the cognitive processing pathways formed between neurons in the brain) and then gradually refines with each forward and back pass of the data.

Radin then used the second half (consisting of RNG data individually produced by the same PEAR participants in a separate PK test) to test the network and see if it could apply the knowledge it had "learned" about the patterns to new sets of data. Compared to when two types of control data-random (consisting of simulated RNG test data randomly generated by a pseudo-RNG) and scrambled (consisting of the actual RNG test data being randomly matched to different participants)—were used in the task, the number of times that the network was able to correctly identify participants was significantly higher $(p<.05)$. Significant results were also achieved in the second study using data from additional PEAR participants.

Though limited in number, these results would seem to offer preliminary suggestion that the idea of there possibly being a "psychic signature" which may be unique to some participants is worth studying further.

Emotional Expression. Some people who claim to have spontaneously experienced PK in their lives have said that their experience seemed to occur at a moment when they felt emotionally stirred; in one such instance, a woman said she felt ". . . a surge of 
blood running through [her] veins" (L. E. Rhine, 1963, p. 99) just before a book seemed to mysteriously fall from a nearby bookshelf. Just before one PK experience occurred, another individual stated that she "... was in a highly emotional state; I'd been in a disagreement with a colleague and didn't feel l'd got my point across. So [I] . . . was very annoyed and frustrated." And before another experience, the same individual said that she felt "sadness, extreme sadness" (Heath, 2000, p. 60). Physical disturbances seemingly occurring in conjunction with periods of emotional turmoil have sometimes been reported in ostensible poltergeist cases, as well (e.g., Auerbach, 1996, Ch. 8; Carrington \& Fodor, 1951, p. 19; Huesmann \& Schriever, 1989; Rogo, 1986; Roll, 1977, 1983). Could this hint at a possible correlation between PK and emotion?

Four experiments were designed by Lumsden-Cook (2005a, 2005b) to further explore this possibility. In the first two, RNG data were collected while artificial mood induction techniques were used in attempt to elicit emotional states in two groups of participants: The first group comprised animal welfare activists, who were asked to recall a particularly infuriating moment in their life and share their reactions to reported incidents of animal cruelty (as a means of inducing feelings of anger). The second group comprised participants who attempted to elicit different mood states (e.g., anger, elation) through a combination of self-referential stimuli (statements worded to give them a sense of feeling a particular mood in themselves), recalling personal moments when they experienced a particular mood, and acting out certain emotional expressions. Although the results with both groups were mixed and not overly significant, some suggestive hints were found of non-random shifts in the RNG output that appeared to be correlated with subjective shifts between emotional states, particularly the release and dissipation of anger.

This suggestive correlation was explored further in the third and fourth experiments, using personal recall and voluntary mood induction combined with exposure to audio-visual stimuli (music, film clips) to elicit emotional states (e.g., sadness, happiness, anger) in two other groups comprising volunteer participants. Although the results with these third and fourth groups were nonsignificant overall, significant deviations from expected randomness $(p<.03)$ were again observed in the RNG output during moments when participants tried 
to release their emotion and shift their mood back toward a calmer, more neutral state.

Three other studies, conducted in a field RNG context, also have obtained results seemingly in line with a possible correlation between PK and emotion: In the first, Blasband (2000) set up an RNG to continuously run in the background while he was in session with clients of his private psychotherapy practice. Several of the clients exhibited strong outbursts of anger and anxiety during the sessions, and these were found to correlate with significant non-random deviations in the RNG output $(z=3.55$ for anger, and $z=-4.47$ for anxious crying; $p<.01)$.

In the second study, Nelson (2008) examined a certain subset of events contained within the formal experimental database of the Global Consciousness Project and found that events which could be rated as having a relatively high degree of emotional impact had a tendency to be correlated with significantly stronger deviations from randomness in the GCP's global-spanning RNG network than events which could be rated as having a relatively medium or low emotional impact $(p \leq .004)$.

In the most recent study, Shimizu and Ishikawa (2012) collected field RNG data while participants viewed three short films, each of which were intended to elicit a specific type of emotion (surprise, sadness, or laughter). When combined, the data from all three films revealed a significant deviation from expected randomness $(z=2.08$, $p=.038$, two-tailed).

Although their results do seem to suggest a possible PK-emotion correlation, it is also important to note that the number of RNG samples collected in most of these studies (e.g., Blasband, 2000; LumsdenCook, 2005a, 2005b; Shimizu \& Ishikawa, 2012) was relatively small, leaving open the possibility that the observed deviations in their output could still be due to inflated chance fluctuations. Additional replications will thus be necessary in order to determine whether similar findings can be obtained with larger samples.

Volitional Strategies. When participants attempt to mentally influence the output of an RNG, by what means do they try to do it? Are there any particular types of strategies that participants tend to use in their efforts to get the RNG output to conform with their willful intent, and which of these strategies (if any) tend to be successful?

One of the initial efforts to explore this was informally made early 
on in the PEAR benchmark program, and it was generally observed that

... individual strategies varied widely. Most simply attended to the $[\mathrm{PK}]$ task in a quiet, straightforward manner. A few used meditation or visualization techniques or attempted to identify with the device or process in some transpersonal style; others employed more assertive or competitive strategies. Some concentrated intently on the process; others were more passive, maintaining only diffuse attention to the machine while diverting their immediate focus to some other activity, such as glancing through a magazine, listening to music, or even eating lunch. One of the more intriguing comments in the experimental logbook was from an operator [i.e., participant] who reported that "Vanilla yogurt really works." Again, little overall pattern of correlation of such strategies with achievement was found. Rather, the effectiveness of any particular operational style appeared to be operator-specific and transitory; what seemed to help one operator did not appeal to another, and what seemed to help a given operator on one occasion might fail on the next. If there was any commonality apparent in this diversity of correlations, it was that the most effective operators tended to speak of devices in frankly anthropomorphic terms, and to associate successful performances with the establishment of some form of bond or resonance with the device, akin to that one might feel for one's car, tools, musical instruments, or sports equipment. (Jahn \& Dunne, 2011, pp. 70-71)

Exploration of the PK volitional strategies employed by participants has also been made in a few qualitative studies (Gissurarson, 1997; Heath, 2000), which have identified a wide range that includes:

— focused awareness or intent

- passive volition or "effortless effort" (mentioned further in the next subsection)

- relaxation

— entering a mildly altered state of consciousness (e.g., meditative states; a feeling of dissociation from one's sense of self identity; letting one's attention become entirely absorbed in the task)

- verbally coaxing the RNG or one's self to produce the desired effect

- mentally visualizing images that reflect one's own concept of the 
PK process, or that would be helpful toward achieving the goal (discussed further in a following subsection)

— having a playful attitude

— eliciting emotional states

- concentrating or staring hard at the PK target

- developing a sense of resonance with the RNG and/or the test computer

— temporarily suspending all disbelief and analytical thinking

— inviting or channeling a form of transcendent "energy"

— frequently releasing (or "letting go" of) one's effort or attention (discussed in the next subsection)

As with the observation initially made in the PEAR program, the strategy that seemed to be most commonly mentioned in these studies was developing a sense of "resonance." An experimental effort to explore this was made by Houtkooper (2004), who asked participants taking part in the Giessen portion of the Mind/Machine Interaction Consortium replication to keep a record of the subjective strategies they used in their attempts to influence the RNG. In line with the test prediction, participants' use of the resonance strategy was found to be significantly associated with (positive) PK scoring $\left(t_{577}=1.889, p=.03\right.$ ), although a closer examination of the data later revealed some suggestive hints of an underlying experimenter effect, in that the

\begin{abstract}
... strong interaction found between the experimenter and the performance with different volitional strategies means that the optimum strategy depends on the person[ality] of the experimenter and, supposedly, the messages the experimenter is conveying, consciously or subconsciously, about the volitional strategies and possible [sic] the whole setting of the experiment (Houtkooper, 2006a, p. 82).
\end{abstract}

Passivity and Release of Effort. Some studies have also explored the passive volition or "effortless effort" strategy (Braud, 1978; Debes \& Morris, 1982; Honorton \& Barksdale, 1972; Roe et al., 2004), in which participants remain relatively relaxed and do not overtly seem to strive much toward intentionally influencing the RNG output. The results of these studies have generally been mixed in terms of significance 
and direction (some have produced positive scoring, while others have produced negative scoring) but the number of them remains very small, indicating the need for more clarifying data.

A somewhat related strategy is release of effort, in which PK effects seem to manifest in the moments after the participants have stopped focusing or exerting their mental intention upon the PK target (and may have also turned their attention away from it). This strategy was explored by Palmer and $\operatorname{Kramer}(1984,1987)$ in two experiments that were designed to have three PK test periods: In the first study, participants concentrated on the RNG and attempted to exert their influence upon it during the first and the third periods, while they were asked to stop concentrating and rest during the second period. The release of effort effect was predicted to occur during the beginning of the latter period, and in line with this, the associated test data were found to be significant $(z=2.30, p<.05)$. The result did not replicate in the second study, however, being nonsignificant and in the direction opposite to prediction $(z=-0.30)$. One potentially relevant difference in this second study is that the PK influence instructions given to the participants could be seen as having conveyed a less effortful suggestion, in that they instead ". . . were instructed to try and merge their consciousness with the computer while focusing on the target number" (Palmer \& Kramer, 1987, p. 129). Some hints of an underlying experimenter effect were also indicated in the data for both studies as well, adding another possible confounding factor. However, the results of another (non-binary RNGrelated) PK experiment (Stanford \& Fox, 1975), in which participants attempted to influence the electrical resistance of a photocell tightly enclosed in a box to shield it from external light, did seem to be in line with a release of effort effect, suggesting that further empirical testing for such an effect should still be pursued in the future.

Visualization Practice. In some spontaneous experiences of ESP, the psychic impressions received by the experient have been reported to subjectively manifest in the form of mental images (Irwin, 1994, Sect 2.2). One might figure that mental imagery could also possibly be involved to some degree in the subjective experience of intentionbased PK, since, as noted by Gissurarson (1992a), “. . . it would seem the assumption behind the importance of imagery for PK is that imagery and visualization play a large role in wishing and willing" (p. 330). 
To explore that possibility, some studies have explored whether the practice of mentally visualizing volition-related images might be an effective strategy for producing PK. Such images may be of two forms: goal-oriented (images which directly or symbolically reflect the intended goal or desired outcome that is being aimed for), or processoriented (images which symbolically reflect a hypothetical process or mechanism by which one could personally conceptualize and envision how PK works, such as by imagining it to be some kind of mental "force" which "pushes" the target in the intended or desired direction).

In reviewing the various experimental studies of this type up to the early 1990s, Gissurarson (1992a) generally found that:

Of the 11 studies reviewed ... eight can be viewed as having explored the role of some form of goal-oriented imagery in the production of PK ... seven of the eight studies yielded PK scores in the expected direction for goal-oriented imagery, and the scores were significantly above chance in three studies in which immediate feedback of performance was provided. ... Hence, it appears that goal-oriented imagery may be important in the generation of PK. (Gissurarson, 1992a, p. 330)

He did note, however, that at least two of the studies did not produce results to indicate that prolonged visualization practice was correlated with an increase in PK scoring.

Has there been any additional evidence gathered since that time to suggest that imagery tends to be associated with PK? At least three experiments have been conducted to further explore this possibility:

Two experiments, conducted by Taylor (1996), were conducted in a practical sporting context, in which two groups of athletes (gymnasts and bore shooters) were trained to engage in a self-paced guided visualization practice that was intended to help improve personal performance in their respective sport. The athletes in each group were divided into two subgroups, experimental and control, with the experimental subgroup undergoing an imagery training period that was twice as long as that of the control subgroup. To see if their imagery practice might be associated with PK performance, each group participated in a computerized PK test that was custom-designed to represent an animated simulation of their respective sporting activity 
(pommel horse vaulting for the gymnasts, and target shooting for the bore shooters), with the actions and outcomes being determined by the output of a (pseudo-random) RNG. Although they did not attain statistical significance, the results of both experiments were in line with the PK-imagery correlation, in that the experimental subgroups produced higher PK scores than control subgroups, with one of the experiments (involving the gymnasts) being suggestive $\left(t_{20}=1.621, p=\right.$ .06). In addition, self-rated changes in imagery ability over the course of the training period were found to be slightly correlated with changes in PK scoring. But as Taylor (1996) importantly noted, the statistical power of this experiment was quite low (owing to the focus of its participant sample being only upon athletes who were committed to the practice of their particular sport), and other possible factors apart from imagery ability such as an experimenter effect and motivational factors might alternately account for the results.

Two experiments conducted by Collesso et al. (2021) were aimed at exploring whether participants' practice of two brief exercises (meditation and guided visualization) might correlate with enhancement of their PK performance. In the second of the two experiments, the participants were asked (prior to engaging in an RNG-PK test modeled after the one used in the PEAR benchmark program) to practice a three- to five-minute guided visualization exercise that was specifically intended to "... stimulate imagination and further induce relaxation as well as positive emotions, including promoting feelings of love" ( $p$. 321). Analysis subsequently revealed a significant deviation from mean expectation in the RNG output $\left(t_{29}=2.66, p=.01\right.$, two-tailed). Moreover, this result was found to be significantly different $\left(t_{58}=-2.69, p=.009\right.$, two-tailed) from the participants' performance in the first experiment (which was designed to act as a control condition, involving the same RNG-PK test with none of the exercises being practiced beforehand). However, due to its being paired with another exercise (meditation), it is unclear how much of this observed effect might have been due specifically to the guided visualization.

Thus, while the results of these two additional experiments do initially seem to be in line with the possible visual imagery-PK correlation, they do not offer unambiguous support for it, and additional clarifying data are still needed at present. 
Meditation. As pointed out by Gissurarson (1992a):

The term "meditation" has been used as a broad label covering different breathing techniques, mental relaxation, and concentration exercises for quieting the flow of thoughts by contemplating a given sound, word, image, thought, or even nothing at all. (Gissurarson, 1992a, p. 310)

A number of experiments conducted since the 1970s have produced results suggesting that meditative practice may be correlated with successful ESP performance (for reviews, see e.g., Honorton, 1977, pp. 437-442; S. Schmidt, 2008), and a smaller amount seemed to offer preliminary evidence suggesting the same for micro-PK (see, e.g., Braud, 1990; Gissurarson, 1992a, pp. 306-311; S. Schmidt, 2008). Does the latter continue to hold for some of the latest RNG-based experiments?

Schmidt and Dalton (1999) conducted one of the most recent studies touching upon this topic, in which they performed three retro-PK experiments involving three separate participant groups who were asked to try and influence pre-recorded sets of RNG data. One of the groups comprised individuals who practiced various forms of meditation. Each participant was asked to listen attentively to auditory tones being played at randomly determined times (derived from the RNG data), with the aim of trying to shorten the time interval between tone soundings. Overall scoring was slightly positive but nonsignificant $(z=0.66)$.

In one of the five experiments reported by Nelson and Schwartz (2006), RNG data were collected over a period of approximately one minute while Nelson engaged in various meditative techniques. A significant positive correlation was found between Nelson's subjective assessment of the depth of his meditative state (coupled with a sense of time distortion) and measures of the absolute deviation from randomness in the RNG output $(r=.458, p<.0001)$.

Thalbourne (2006) initially conducted five experiments designed to explore possible deviations in RNG output occurring in conjunction with the practice of eliciting Kundalini (the "Eastern name given to a particular syndrome of psychophysiological changes taking place in 
the body, often as a result of long practice of meditation or yoga"; Thalbourne, 2008, p. 155). Acting as his own participant, Thalbourne attempted to elicit sensations of Kundalini "energy" through various techniques including mental absorption in music, mental attendance to a theological poem through repeated recitation, and focus on somatosensory "noise" (said to be sometimes associated with Kundalini). With the exception of one experiment where the results of both test runs had negative scores, there was a suggestive tendency for Kundalini-sensation runs to produce positive scores and nonKundalini runs to produce negative scores. Thalbourne (2008) later attempted to replicate these findings in two additional experiments, but the results were opposite to prediction, with non-Kundalini runs resulting in positive scores, and Kundalini runs being near chance. The possibility that some of these results may have been due to a visual feedback artifact (associated with the display lights on the face of the RNG device) has been examined and found to be unlikely (Thalbourne \& Storm, 2015).

In one of their two experiments, Collesso et al. (2021) asked participants to engage in two brief exercises, one of which was a two- to three-minute meditation exercise intended to induce bodily relaxation and the release of tension, before attempting to influence the output of an RNG. Results indicated a significant deviation from mean expectation $\left(t_{29}=2.66, p=.01\right.$, two-tailed), and when compared with the results of the initial (control) experiment where these exercises were not used, a significance difference was observed $\left(t_{58}=-2.69, p=\right.$ .009 , two-tailed). As mentioned, however, due to its being paired with another exercise (guided visualization), it is unclear how much of the observed effect might have been due specifically to the meditation.

Other recent experiments examining the possible effects of meditation have been conducted in a field RNG context: Radin et al. (1996) had set up an RNG to continuously run in the background over the course of an all-day workshop devoted to the practice of Holotropic Breathwork, an approach which is intended to induce altered states of consciousness using a combination of spiritual insights and techniques (including the breathing techniques of pranayama meditation). The resulting data exhibited a significant deviation from expected randomness $(z=2.96, p=.002)$, whereas control data collected after the 
workshop was over were consistent with chance $(z=-0.979)$.

Mason et al. (2007) set up two field RNGs to run continuously during a series of Transcendental Meditation sessions held over the course of a 96-hour period at the Maharishi University of Management (MUM) in lowa. In addition, 149 hours of data were separately collected from the RNGs before and after the series, when they were left to run continuously in a university office as a control setting. Data from each of the two experiments conducted during the MUM series were found to exhibit strong deviations from randomness in the negative direction $(Z=-4.726, p=.000001$; and $Z=-3.872, p=.00005)$, while control data taken before the series were consistent with chance. In addition, an unexpected result was found with the control data taken after the MUM series, in that these data also exhibited a very strong and persistently negative deviation $\left(Z=-7.28, p=1.70 \times 10^{-13}\right)$. Several possible ways to account for the latter were considered, including the possibility that it might represent a kind of "lag" or "carry over" PK effect based on the consideration that "... previous studies with this type of meditation had reported a carryover or lag effect on the experimental measurements even after the experimental period of meditation had ended" (p. 307). However, it is also important to note that such isolated instances of strong and persistent deviations in a certain direction are also sometimes observed in RNGs which are malfunctioning, leading them to produce a consistent artificial bias in their output. Although no clear evidence for malfunctioning was found, it would seem that this possibility cannot be ruled out with complete certainty, thus leaving a potential confound in the interpretation of this finding. However, it does provide an instructive example of what researchers should be watchful for, and be cautious of, when trying to interpret extremely deviant results.

Similar in aim to the studies by Thalbourne (2006, 2008), Ivtzan (2008) explored a possible micro-PK correlation with Kundalini practice by collecting data from two field RNGs during a series of 60-minute sessions held over the course of five weeks with ten practitioners of a form of meditation intended to elicit Kundalini. One RNG was located in the meditation room, while the other was located 2 kilometers (1.24 miles) away. Half of the practitioners were made aware of the presence of the first RNG in the room during the sessions, while the other 
half was kept unaware of its presence (by hiding the RNG in a bag). RNG data collected in the presence of the "aware" group exhibited a significant positive deviation $(z=2.48, p=.006)$, while data collected in the presence of the "unaware" group were nonsignificant. Data from the second (distant) RNG were found to be nonsignificant, as well.

In line with previous findings (Braud, 1990; Gissurarson, 1992a, pp. 306-311; S. Schmidt, 2008), the majority of the latest experiments continued to give results suggestive of a correlation between meditation and deviations from randomness in RNG output.

Healing Intention. Apart from physical targets like RNGs, a number of PK experiments have also been focused on the question of whether a participant can mentally influence small-scale biological targets such as cell cultures, electrodermal activity, shifts in human attention, plant growth, and animal locomotion (Braud, 2003; Delanoy, 2001). Meta-analyses of these experiments do tend to show a small but significant overall effect (Roe et al., 2015; Schlitz \& Braud, 1997; S. Schmidt, 2012; Schmidt et al., 2004), which may provide a possible basis for conceptualizing ostensible psychic healing. One question which arises in the latter context is: Do these biological PK and ostensible healing effects involve a similar (if not the same) kind of ostensible influence process as PK effects upon physical targets? One reason for considering this possibility from a conceptual viewpoint comes from observations made of some of the subjective factors that seem relevant to the healing experience, a few of which seem suggestive of willful influence in that a healer consciously or subconsciously "must affirm the idea or intention to help people" and "direct one's attention in a positive direction" (Levin, 2011, p. 22). Another comes from an observation made by Nelson (1999):

Living bodies, with homeostatic, immune, and nervous systems that epitomize the realm of applied nonlinear dynamics, are intrinsically susceptible to influence from small inputs and are able to identify and amplify the most subtle of inchoate patterns and information. Biological systems utilize random processes and uncertainty to maintain the highest level of sensitivity to subtle changes in the environment. They are reactive on the finest scale to information that reduces entropic disorder and provides an increment of structure and predictability, yielding a stable internal milieu and 
successful interaction with the environment. In this context, we see that healing a wound or recovering from an illness is dependent upon the generation or addition of appropriate information to help restore order and structure. (Nelson, 1999, pp. 20-21)

A relatively small number of studies, mostly utilizing a field RNG approach, have taken preliminary steps toward examining this question empirically (Nelson \& Radin, 2003).

Schwartz et al. (1997) collected RNG data over the course of a large three-day meeting devoted to the traditional Chinese practice of Qigong and the study of its possible beneficial effects for human health, based partly on the claim that "Qi accumulates over the entire meeting" (p. 57). The resulting meeting data were found to exhibit a significant increase in the mean number of trials that were above chance expectation $\left(t_{4}=3.56, p<.023\right)$, whereas such increases were not found in control data collected over an equal number of days before and after the meeting.

Crawford et al. (2003) conducted two experiments in which they statistically compared two sets of RNG data that were collected in two different settings over the course of 61 days: The experimental setting was the treatment room of a healer's office where non-contact bioenergy treatments were being given to patients on a regular basis, and the control setting was a local university library. The combined results indicated that there were significantly more instances over the course of the 61 days in which the experimental RNG data exhibited notable deviations from expected randomness (at or exceeding the $p$ $<.05$ level) as compared to the control data $\left(X^{2}=16.3,1 d f, p<.0004\right)$.

Radin et al. (2004) conducted a three-day experiment in which they continuously collected data from three separate types of RNGs before, during, and after a small group of healers attempted to affect the growth of cell culture targets and "condition" the space of the treatment room using the traditional Japanese spiritual healing practice of Johrei. Upon combining the data together, a highly significant deviation from expected randomness was observed across all three RNGs on the morning of the third day of the experiment $(Z=4.80, p=.00009)$.

Two more RNG experiments were independently conducted with Johrei practitioners by members of the PEAR staff (Jahn et al., 2006). 
The first was a "Yantra" experiment in which the audio (rhythmic drumbeats) and visual (a mandala design that dynamically changes colors on a computer screen) aspects of the test environment are coupled to the output of an RNG (Dobyns et al., 2007). It differed from the standard "benchmark" PK test in that no real-time feedback was given to the participant on their performance during the test, and it consisted of only the "HI" and "LO" conditions (i.e., the "BL" condition was omitted). The three practitioners took part in the experiment both while engaging in Johrei practices and also while not; subsequent comparison of these two conditions indicated notable differences in their outcomes: Whereas the "HI-LO" difference from the non-johrei condition was found to be at chance $\left(Z_{\text {diff }}=0.387, p=.70\right)$, the "HI-LO" difference associated with the Johrei condition was significantly in the direction opposite to intention $\left(Z_{\text {diff }}=-2.756, p=.006\right)$.

Lumsden-Cook et al. (2006a) conducted an exploratory study in which South African native Zulu healers were asked to try and influence the output of an RNG using the same ritual healing practices that they perform on human patients. A significant positive shift away from mean expectation was observed across the combined data from all four healers $(Z=2.531, p=.011$, two-tailed). This outcome prompted a second, follow-up study (Lumsden et al., 2006b) with a larger test sample of 20 healers who were again individually asked to try and influence an RNG in a healing context. This again resulted in an overall significant outcome $\left(X^{2}=113.02,80 \mathrm{df}, p=.009\right)$ that differed from the first in that it was non-directional (i.e., both extreme positive and negative deviations from expectation among the test trials were collectively taken into account, regardless of their polarity or sign).

In a recent preliminary experiment, Moga (2015) collected RNG data during a series of 18 sessions conducted with five Healing Touch practitioners. Data from seven of these sessions were found to exhibit deviations from randomness exceeding the $p=.05$ level, and these occurred in sessions with three of the five practitioners.

While the findings of several of these studies do seem to indicate possible influence effects upon the RNGs, the number of them remains relatively small at this point. In addition, some of them (Lumsden-Cook et al., 2006a, 2006b; Moga, 2015) collected a relatively small amount of RNG test samples (e.g., the duration of the test periods employed by 
Lumsden-Cook et al. were only about five minutes in length) and/or seemed to base their conclusions on the outcome of only a relatively small number of sessions. Considering the relatively small effect sizes associated with micro-PK, it can be difficult (with such small samples) to reliably establish the presence of a micro-PK effect upon the RNG output that is clearly distinguishable from expected chance fluctuations. Thus, additional data will be necessary in order to further verify the significant deviations observed in these studies.

Attitudes Toward and Personal Experiences of PK. One finding that has appeared fairly consistently across a number of experiments on ESP is that people who hold some degree of personal belief in this type of phenomenon also tend to score well in tests of it (Lawrence, 1993; Schmeidler \& McConnell, 1958; Storm \& Tressoldi, 2017). Is there a similar kind of correlation between belief and PK performance?

As part of their effort to explore this question (among others), Gissurarson and Morris (1991) conducted five experiments in which participants' responses on several personality questionnaire items were compared with their subsequent scores on a PK test. One of the items inquired about their level of belief in PK, and although one of the experiments did result in a significant positive correlation (Spearman's $r h o=0.46, z=2.90, p<.005$, two-tailed), it was not persistently observed across the other four. A broader meta-analysis by Gissurarson (19901991) of 450 experimental test sessions conducted between 1946 and 1991 further indicated only a very modest and suggestive correlation at best (Spearman's $r h o=0.19, z=1.38, p=.08$, two-tailed). Thus, the answer remains somewhat unclear and further clarifying data are needed.

One particularly notable finding to emerge from these two studies was an indication that participants who reported previously having a spontaneous PK experience had also exhibited a tendency to score well on the PK test. Combined results across the five experiments by Gissurarson and Morris (1991) revealed a significant positive correlation (Spearman's rho $=0.27, z=3.03, p=.001$ ) that remained fairly consistent in the meta-analysis by Gissurarson (1990-1991) that followed (Spearman's rho $=0.22, z=3.15, p=.0008$ ).

Though the amount of data is currently limited, these findings would seem to offer some initial hints for further examining the possible relevance of attitudes and personal experiences to PK performance. 


\section{Neuropsychological Factors}

Cerebral Lateralization. Although there are some caveats, it has long been thought that the two hemispheres of the human brain generally tend to be specialized for certain cognitive capacities and modes of thinking: The left hemisphere has often been seen as the "seat of language" and as mostly being characterized by sequential, analytical, and rational thought; while the right hemisphere has been seen as the main area involved in controlling visuospatial skills and as mostly being characterized by non-verbal, non-analytical, and intuitive thought (Kolb \& Whishaw, 2006, pp. 542-550; Springer \& Deutsch, 1998). One might wonder: If psi is a genuine human capacity, then might it be associated with a particular hemisphere? While the current empirical evidence is not overly clear on this matter, there would seem to be some modest indication that ESP may be associated more with the right hemisphere (C. H. Alexander, 2002; Broughton, 1983; Williams, 2015, Ch. 8). What about for PK?

Initial hints of a similar association would seem to come from subjective accounts given by individuals who have reportedly experienced PK; for instance, one individual stated:

I think the common thread ... is that the cognitive left brain analyzing part of the mind is out of the picture for a while, either through my getting very emotional, or deliberately occupying that part of my mind with something else. So I think getting that cognitive, verbal part that said, "oh, you can't do it," just disabling that, or putting it out of the way for a while seems to help. (Heath, 2000, p. 59)

The relatively few attempts that have been made so far to address the matter using an empirical approach would seem to offer further hints, as well: Prior to engaging in a RNG-PK test, Andrew (1975) had two groups of participants listen to a brief relaxation audio tape, followed by a 23-minute long tape which emphasized a particular mode of thought: One group heard a "left thinking" tape that presented material oriented toward a verbal, analytical, and rational mode (such as vocabulary and grammatical lessons, mathematical and logical problems, and readings in philosophy, law, and physics), while the other group heard a "right thinking" tape containing material oriented 
toward a spatial and non-analytical mode (such as music and sounds which were meant to subjectively evoke imagery and convey a sense of depth). Results of the subsequent PK test indicated that the "right thinking" group tended to score positively (binomial $p=.02$ ), while the "left thinking" group tended to score negatively $(p=.011)$, leading to an overall significant difference between them (Mann-Whitney $U=$ 8.5, $p<.002$, two-tailed). Two follow-up experiments by Braud et al. (1976) produced somewhat similar results, and when the data from all three experiments were combined, there was a tendency for the "right thinking" group to produce significantly positive scores (binomial $p=$ .025), and the "left thinking" group to either produce negative scores or null results, with a significant difference again being indicated $(p<.01)$.

To basically assess the degrees to which they may have engaged in "left thinking" versus "right thinking," Krieger (1977) asked each of her participants to complete the "similarities" test from the Wechsler Adult Intelligence Scale (a test which assesses logical, abstract thinking by asking participants to analyze the relational features between two objects; Cohen et al., 1996, p. 306), as well as the Street visual Gestalt test (which assesses visuospatial thinking by asking participants to mentally recombine scattered visual elements together in order to perceive a whole object). A ratio was then calculated from the scores of these two tests to provide a numerical indicator of the relative engagement level of these two modes of thought. The participants were then tested for PK under two conditions: In the intention condition, they were presented with a target lamp that flashed on or off based on the output of an RNG, and they attempted to keep the light on for as long as possible. In the non-intention condition, the lamp was placed in a separate room and the participant was left unaware of its presence there. Statistical comparison of the participants' ratios with the scores they achieved in the two PK test conditions revealed a fairly even degree of correlation between them (intention: $r=-0.44, p<.02$; non-intention: $r=-0.43, p<.02$ ). In particular, a significant negative correlation was found between their intention PK scores and their performance on the "right thinking" task $(r=-0.53, p<$ $.01)$, and a significant positive correlation was found between their nonintentional PK scores and their "left thinking" task performance $(r=0.37$, $p<.05$ ), suggesting that intentional and non-intentional (or unconscious) PK were associated with the right and left hemispheres, respectively. 
Certain studies using electroencephalographic (EEG) and neuroimaging technology have produced a few other findings relevant to the question of whether micro-PK might be associated with a particular brain hemisphere; these will be described in the subsections to follow.

EEG Activity. To explore whether PK performance might be correlated with a particular type of brain wave activity, some experiments have sought to actively monitor participants' EEGs while they are engaged in an RNG-PK test. Several of these were designed to have a neurofeedback-type approach, in which the RNG was coupled to an EEG monitor and would become activated whenever a specifically defined brain wave frequency or voltage threshold was reached.

Two experiments of this type by Schmidt and Terry (1977) involved monitoring brain wave activity from the participants' occipital lobes, and the RNG was activated only whenever the EEG monitor registered activity in the alpha frequency range $(8-12 \mathrm{~Hz})$ during one half of the PK test sessions, and in the beta frequency range $(13-30 \mathrm{~Hz})$ during the other half. Upon being activated, the RNG began generating binary output that was converted to a random sequence of high- and lowpitched tones, and these were presented to the participants with the intended PK goal of producing more low tones. An overall success rate of $50.7 \%(z=3.12, p<.001)$ was achieved, with the scoring being distributed across both the alpha and beta sessions.

A series of experiments by Heseltine (1977) utilized a similar PK testing setup in which an RNG was coupled to an EEG device that monitored fluctuations in voltage across successive brain wave cycles. Each time the zero point (going from negative to positive voltage, or vice versa) was crossed in the course of a wave cycle, the device initiated a test trial by triggering a digital flip-flop in the custom-made RNG, momentarily halting it and thereby causing a binary bit outcome to be randomly determined for that trial. If the outcome matched the binary number selected as the target by the controlling computer, a success was registered for that trial. The computer continually monitored the trial outcomes over the course of a three-second period, and using a majority-vote process (based on how many successes were registered over that period), it determined whether auditory feedback (in the form of a high- or low-pitched tone) would be presented to the participant; 
the more successes that were registered, the longer the tone would persist. Participants were asked to listen for the tone but to try and influence it (by extending its length for as long as possible) only in a casually passive manner while letting their mind wander freely.

Results of the first experimental series indicated that participants tended to be successful at the PK test when the low tone served as the feedback target ( $50.8 \%$ success rate, $z=2.48, p=.014$ ), while test trials with the high tone were at chance. The low tone was thus kept as the target in the second series, which again resulted in significantly positive results ( $50.4 \%$ success rate, $z=3.53, p=.0004$ ). Spectral analysis further indicated that scoring tended to be highest when the participant's EEG was primarily within the alpha frequency range $(z=3.60, p \leq .0005$, two-tailed) and, to a lesser extent, in the beta range $(z=2.10, p \leq .04$, two-tailed).

Three more experiments in this series by Heseltine and MayerOakes (1978) explored possible brain hemisphere correlations by collecting EEG data over the left frontal and parietal regions of the participants' brains in Series 3 and 5 and comparing RNG-PK performance with the EEG data that were collected over the participants' right frontal and parietal regions in Series 2 and 4 . The combined data across Series 2 and 4 resulted in significantly positive scoring $(50.3 \%$ success rate, $Z=3.04, p=.002$ ), whereas the combined data for Series 3 and 5 resulted in significant negative scoring ( $49.8 \%$ success rate, $Z$ $=-2.29, p=.025)$, suggesting that the right and left hemispheres were associated with positive and negative PK scoring, respectively. Spectral analysis indicated that the highest scoring was again associated with the alpha frequency range $(z=2.31, p \leq .025)$ for the right hemisphere, whereas no clear correlations with frequency range were found for the left hemisphere.

Though they are limited in number, these experimental findings would seem to suggest that micro-PK tends to be most often correlated with alpha wave activity, a finding paralleled in experiments on ESP (for reviews, see, e.g., C. H. Alexander, 2002; Krippner \& Friedman, 2010; Williams, 2015). Further attempts at verification of this with a broader participant pool would be useful in better determining whether this may indeed be a robust finding and whether it might be generalizable to a wider population. 
PK Correlates of Bodily Motor Volition. In pondering the processes that might underlie the initiation of bodily movement, Eccles (1977) suggested that perhaps PK might play a minor functional role in the interaction between mind and brain "... when you will a movement and you actually trigger the brain to carry out the movement; using thought to bring about action" (p. 256). His basic premise was that, in order to convey its intent to move and thereby get the brain side of the process started, the mind might subtly exert a willful influence upon the electrochemical functions of one or more neurons contained in the areas of the brain involved in motor control. A potential target that Eccles specifically suggested for this influence is the functioning of the synaptic bouton, a bulbous cellular structure found at the ends of the neuron's branching arms (called teleodendria) which contains bundles of neurotransmitters that the neuron releases into the narrow synaptic gap to establish communication (through chemical receptor signaling) with adjacent neighboring neurons. Electrical excitation pulses received via the teleodendria help facilitate activation of each bouton and the subsequent release of its neurotransmitters, with an estimated "all-ornothing" occurrence probability of about $50 \%$. To initiate the movement process, perhaps the mind might willfully influence this probability to a degree higher than 50\% (Beck \& Eccles, 1992; Eccles, 1986).

Might Eccles' PK-related suggestion be plausible in any way? In examining this from a theoretical standpoint, Helfrich (2007) pointed out that this probabilistic electrochemical functioning of neural synapses can be viewed as being analogous to the generation of binary bits from an RNG, and through statistical reasoning he argued that the small effect sizes commonly found in RNG-PK studies might actually be of the right size to affect this probabilistic functioning.

Certain findings also seem to offer some preliminary support from an empirical standpoint, as well: In two biofeedback-type experiments designed to test Eccles' (1977) hypothesis in a basic manner, Honorton and Tremmel (1979) asked participants to attempt to mentally influence their own brain wave activity such that it would begin exhibiting rhythms in the alpha frequency range. A tone would be presented to the participants as auditory feedback by an EEG monitor whenever it detected the presence of alpha rhythms, and the aim of the participants was to keep this tone on for as long as possible. Data from an RNG 
running hidden in the background were found to exhibit significant deviations from chance in both experiments (Expt. 1: $X^{2}=145.7,100$ $d f, p=.002$, two-tailed; Expt. $2: X^{2}=159.2,120 d f, p=.005$ ) while the participants were successful at this EEG influencing task. In contrast, no significant deviations were observed when participants were unsuccessful or were simply resting and not focused on the task.

Although they differed from the aforementioned one in several respects, a series of conceptually similar multi-factorial experiments by Varvoglis and McCarthy (1986) had found significant deviations from chance in the output of an RNG not only when participants were focused on directly influencing the RNG and were receiving real-time feedback on their performance $(z=2.46, p=.007)$, but also when the RNG was hidden and participants were instead focused on trying to influence their brain waves to produce alpha rhythms and were receiving feedback on their performance from an EEG monitor $(z=2.59, p=.005)$. However, necessary adjustments made to the EEG feedback procedure to improve control conditions in these experiments had made it unlikely that this latter result could be fully attributed to the participants' brain wave influence efforts.

Giroldini (1991) made a separate attempt to test Eccles' hypothesis by asking participants to try to influence the output of a custom-designed random generator that was intended to digitally simulate the electrical pulse activity of a simple network of neurons. The experimental data were found to exhibit a small positive shift on the order of nearly $1 \%$ (0.764\%) that was highly significant $(p<.00001)$ and in line with the participants' intention, whereas the non-intention control data were close to zero (-0.06\%) and nonsignificant overall. Compared to control data, EEG data collected from the participants' frontal lobes during the experimental period were found to have higher mean amplitudes in both the alpha and beta range $(p<.001)$. Significantly increased brain wave activity in the alpha and beta bands also was found by Giroldini and Pederzoli (2021) in a conceptually similar PK experiment conducted three decades later using another custom-designed random generator, and this EEG activity was also found to extend into the gamma $(30+\mathrm{Hz})$ frequency range.

Frontal Lobe Correlates. In their independent effort to conceptually replicate the PEAR benchmark experiment, Freedman et al. (2003) conducted an experiment in which two groups of participants-one 
consisting of healthy staff members of a local hospital, and the other consisting of neurological patients-were asked to attempt influence upon the output of an RNG similar in design to the benchmark-type RNG, with visual feedback on their performance being given by the horizontal back-and-forth motion of an arrow on a computer screen (with the head of the arrow pointing in the target direction being aimed for). The test paralleled the standard test procedure utilized by PEAR in having three experimental conditions: "Right" (attempting to move the arrow toward the right of the screen; corresponding to the "HI" condition of the PEAR procedure), "Left" (attempting to move the arrow toward the left; corresponding to "LO") and "Baseline" (not attempting to influence the arrow at all). The resulting test data indicated that while most of the staff members and patients only produced outcomes consistent with chance, there was one patient (a male who had suffered damage to his left frontal lobe) who produced a significant result $\left(t_{5998}\right.$ $=-3.169, p=.0015)$ in the "Right" influence condition. The result was replicated when the patient was tested again in a follow-up experiment $\left(t_{37998}=-2.53, p=.0115\right)$.

The same directional influence was observed when the patient was tested again in a third experiment (Freedman et al., 2018), which also resulted in a second neurological patient (a female who suffered considerable tissue loss in her frontal lobes due to dementia) who was also moderately successful at the same PK test $\left(t_{35998}=-2.16, p=.03\right)$ being identified. These findings led the researchers to "... suggest that the frontal lobes, and in particular the left ... middle frontal region ... may act as a biological filter to inhibit mind-matter interactions" (p. 83; see also Freedman, 2010).

Although their results are intriguing, the number of test trials collected with these successful patients is still relatively small (especially compared to the PEAR benchmark database), and additional replications with larger samples would be useful for further evaluation.

\section{MINDING THE EXPERIMENTER PSI EFFECT}

Arguably, the general review of micro-PK that has been provided here would not be complete without at least a brief consideration of the experimenter effect, as it is a potentially confounding factor which 
is difficult to avoid in experimental PK (as well as other psi) tests, and which may be one of the factors possibly contributing to the challenging situation of replicating findings across parapsychological experiments (as it may offer one way to possibly account for why many of the individual findings tend to be so mixed and inconsistent with others at times).

Although it is often assumed that the ultimate source of any successful performance observed in experimental psi tests is likely to be the participants (since they are the ones being purposely tasked with the effort of trying to mentally influence an RNG or correctly perceive a hidden target using ESP), the possibility has also been recognized that some successful outcomes might alternately be traced to the experimenter(s) running the tests. As White (1976) described, this would be a scenario in which the participant in a test of ESP

... may respond to the wishes and needs of the experimenter over
and beyond what he [or she] is told by the experimenter and under
conditions which rule out of the possibility of sense-mediation. In
such cases, the only way the [participant] could obtain the relevant
information would be from the experimenter by means of telep-
athy (i.e., a psi-mediated experimenter effect), or by clairvoyance
of the testing situation (i.e., a psi-mediated experimental effect).
(White, 1976, p. 334)

In the case of a PK test, a psi-mediated experimenter effect scenario would entail the experimenter (consciously or unconsciously) using his or her own PK ability to successfully affect the RNG output, instead of the participant; while a psi-mediated experimental effect scenario would presumably entail a process akin to the one basically proposed in Decision Augmentation Theory (DAT; May et al., 1995a, 1995b), where instead of mentally influencing the RNG, the experimenter may use precognition to predict and decisively select out ideal periods during the test session when the RNG output is exhibiting brief, non-random fluctuations purely by chance, which then cumulatively amount over multiple sessions to a significant result (for possible counterarguments to this, see, e.g., Dobyns, 2000; Dobyns \& Nelson, 1998). As White (1976) further stated, in such scenarios ". . . it is difficult to see how the experimental situation can be separated from the experimenter, for in 
a sense it can be viewed as a trap which the experimenter has devised with the intention of catching a particular finding which will fulfill his [or her] hopes and expectations" (p. 334).

While a good amount of anecdotal and empirical evidence for it has accumulated over the years (for reviews, see, e.g., Kennedy \& Taddonio, 1976; Palmer, 1997; Palmer \& Millar, 2015; Parker \& Millar, 2014; White, 1976, 1977), the extent to which the experimenter psi effect may pose a serious confounding issue for the interpretation of experimental results continues to be a topic of debate. Apart from those briefly cited in previous subsections, one illustrative example relates to the debate surrounding the interpretation of the significant outcome for the formal 500-event database collected by the Global Consciousness Project (Nelson, 2015, 2019; Nelson \& Bancel, 2011): Although one possible interpretation of this outcome might be that it reflects a micro-PK-related shift away from expected randomness occurring across the RNG network nodes in conjunction with the mass attention and emotion being focused on certain events and activities in the world, there are also two other alternate interpretations that have been proposed: Perhaps the experimenter(s) responsible for defining the time period of examination surrounding each event or activity are (unconsciously) using precognition to predict and select out which particular events and/or time periods will exhibit short-lived non-random fluctuations in the RNG network data purely by chance (Bancel, 2017a, 2017b; May \& Spottiswoode, 2011a, 2011b), or (in the case where the examination period is defined after the event happens) the experimenter(s) are using a form of retro-PK to intentionally influence the RNG network data after they have been recorded and archived (H. Schmidt, 2009). A number of points have been raised to possibly counter these alternate interpretations (Bancel, 2011; Nelson, 2011, 2017, 2020; Nelson \& Bancel, 2009), though the issue continues to remain open and unresolved.

At the very least, in light of the persistence of this issue, it is important for novice investigators and researchers to be mindful of the relevance of the experimenter effect to the procedural design, results, and interpretation of any experiments they may look to undertake in the future. Parker and Millar (2014) and Bierman and Jolij (2020) have recently offered some starting points for consideration in this regard. 


\section{CONCLUSION}

From the review given here, it should be clear that, although a range of proof-oriented empirical evidence for microscopic psychokinetic effects upon RNG output has accumulated over the past eight decades, there is still very little that is known at present about the processes that may possibly underlie them. This might be partly attributable to the following:

— In relative contrast to that which has been done in relation to ESP, a considerably lesser amount of process-oriented research has been conducted in relation to PK. As a result, few attempts have been made to independently replicate and expand upon the preliminary process-oriented studies that have been conducted so far.

— Considering the very small effect sizes that tend to be associated with micro-PK effects on RNGs, it is possible that several of these studies did not have sufficient statistical power with which to detect such subtle effects and thereby perform adequate tests for correlation. Although it tends to be a prime focus for purely prooforiented studies of psi (Tressoldi, 2012), this issue concerning statistical power also tends to be important for process-oriented studies as well, because in order to be able to study the possible correlates of psi, one must first have a reasonable degree of confidence that psi is present to begin with.

This present state of affairs suggests that there is still much opportunity for novice investigators and cross-disciplinary researchers to make valuable contributions in this particular domain of study, and it is hoped that the experimental findings reviewed here can serve as a starting guide in paving the way forward for any future research efforts they may make. It should also be pointed out that valuable insight can potentially come not only from further collection and analysis of quantitative data relating to the purely physical side of PK (e.g., RNG output and numeric-scale instrumental readings), but also from collecting and analyzing more qualitative data relating to its subjective side as well, for although some preliminary effort has been made over the years (e.g., 
Gissurarson, 1997; Heath, 2000), Irwin (1994) has previously observed that: "The phenomenology of ... PK experiences clearly has not been adequately documented" (p. 32).

With further progress along these lines, we may gradually come closer to gaining better knowledge about the relation between mind and matter.

\section{NOTES}

1 Since it seems that RNG tends to be the more frequently used acronym in parapsychology, it will be conveniently adopted for use throughout the rest of this paper.

2 RNGs with a similar conceptual design are also described by Vincent (1970), H. Schmidt (1977), and Aguayo et al. (1996).

3 It should again be emphasized that the RNG trial output example graph shown in Figure 2 only reflects an ideal illustrative example of how the RNG output should be expected to look when roughly conforming to purely random behavior (i.e., random fluctuations around MCE). RNG output does not always conform this closely to the ideal, as there can be some degree of variability in the random noise inherent in an RNG's output which can lead the data to slightly "drift" away from MCE (whether upward or downward) purely by chance. Although such "drifts" tend to be relatively short-lived, they can at times be persistent enough to approach (and even exceed) the significance threshold for a brief time, again purely by chance. Over the long run, however, the output of suitably random RNGs tends to stay somewhere below this threshold. The graph shown in Figure 2 is merely meant to serve here as an ideal illustrative example for purposes of comparison with the PK graphs presented elsewhere in this paper.

4 Based on the proportion index ( $\pi$ ), an effect size estimate developed by Rosenthal and Rubin (1989, 1992-1993) which is conceptually intuitive in that it "... shows the proportion correct, or hit rate, on a scale on which .50 [i.e., 50\%] is always the null value" (Rosenthal \& Rubin, 1989, p. 332); see also Bösch et al. (2006a, p. 501).

5 The reduction in the number of experiments (as compared to 1989) was largely due to 258 experiments from the PEAR program being statistically condensed down to a single data point. 
6 The reduction in experiment number was again due to the data from the PEAR experimental series and the Mind/Machine Interaction Consortium study being condensed down to smaller individual data points.

7 This success rate is based on a converted value of Radin's (2006, p. 323) reported weighted effect size to the proportion index (Note 4), using the conversion $\pi=0.5[$ effect size $]+0.5$ (Bösch et al., 2006a, p. 499).

8 It should be mentioned that Kugel (2011) had reportedly found a number of possible issues with the Bösch et al. (2006a) meta-analysis, which could potentially confound interpretation of its results. These issues include: the inclusion of data from more than 30 ESP tests in what was meant to be a purely PK database; using arbitrarily defined criteria for determining which studies would be included or excluded from the meta-analysis; and suggestive instances of faulty data coding. The results of this meta-analysis should perhaps be approached and interpreted with some degree of caution for this reason.

9 Readers might initially notice a potential inconsistency here, in that Bösch et al. (2006a) had reported this correlation as being positive in the text of their paper (which seems to have stemmed from the way they coded the variables in their assessment). However, they state for clarification that it is ". . . indicating that lower quality studies produced larger effect sizes" (pp. 507-508), which would imply a negative correlation.

${ }^{10}$ Readers may notice that the statistical result in this case is reported as a $T$-score rather than a Z-score, which was done because the instability of the high-speed noise source tended to cause the trial output of the RNG to deviate somewhat from its expected binomial distribution. But as Dobyns et al. (2004) point out, since the associated degrees of freedom "... are in the range 104 to 105 ... the T-distribution differs negligibly from the standard normal distribution" (p. 373), allowing the two types of scores to be considered relatively comparable in this case.

"1. This is because Hansen (2006) has previously pointed out a potential statistical issue with Stevens' (2005) study that still may require addressing. 


\section{REFERENCES}

Aguayo, R., Simms, G., \& Siegel, P. B. (1996). Throwing nature's dice. American Journal of Physics, 64, 752-758.

Akers, C. (1987). Parapsychology is science, but its findings are inconclusive. Behavioral and Brain Sciences, 10, 566-568.

Alcock, J. E. (1987). Parapsychology: Science of the anomalous or search for the soul? Behavioral and Brain Sciences, 10, 553-643.

Alcock, J. E. (1988). A comprehensive review of major empirical studies in parapsychology involving random event generators or remote viewing. In D. Druckman \&J. A. Swets (Eds.) Enhancing human performance: Issues, theories, and techniques-Background papers (pp. 602-719). National Academy Press.

Alexander, C. H. (2002). Psychic phenomena and the brain: An evolution of research, technology, and understanding. Proceedings of Presented Papers: The Parapsychological Association 43rd Annual Convention (pp. 9-24). Parapsychological Association.

Alexander, K. (2019). A multi-frequency replication of the MegaREG experiments. Journal of Scientific Exploration, 33, 435-450. https://dx.doi. org/10.31275/2019/1278

Alvarado, C. S. (2006). Neglected near-death phenomena. Journal of Near-Death Studies, 24, 131-151.

Andrew, K. (1975). Psychokinetic influences on an electromechanical random number generator during evocation of "left-hemispheric" vs. "righthemispheric" functioning. In J. D. Morris, W. G. Roll, \& R. L. Morris (Eds.) Research in parapsychology 1974 (pp. 58-61). Scarecrow Press.

Auerbach, L. (1996). Mind over matter. Kensington Books.

Babu, S. (1987). Analysis of variance of REG data. In D. H. Weiner \& R. D. Nelson (Eds.) Research in parapsychology 1986 (pp. 1-5). Scarecrow Press.

Bancel, P. A. (2011). Reply to May and Spottiswoode's "The Global Consciousness Project: Identifying the Source of Psi." Journal of Scientific Exploration, 25, 690-694.

Bancel, P. A. (2015). An analysis of the Global Consciousness Project. In D. Broderick \& B. Goertzel (Eds.) Evidence for psi: Thirteen empirical research reports (pp. 255-277). McFarland.

Bancel, P. A. (2017a). Searching for global consciousness: A 17-year exploration. Explore: The Journal of Science \& Healing, 13, 94-101. https://dx.doi. org/10.1016/j.explore.2016.12.003

Bancel, P. A. (2017b). Response to Nelson's “Weighing the Parameters." Explore: The Journal of Science \& Healing, 13, 106-107.

Batcheldor, K. J. (1966). Report on a case of table levitation and associated phenomena. Journal of the Society for Psychical Research, 43, 339-356.

Beck, F., \& Eccles, J. C. (1992). Quantum aspects of brain activity and the role of con- 
sciousness. Proceedings of the National Academy of Sciences USA, 89, 11357-11361. Berger, R. E. (1988a). In search of "psychic signatures" in random data. In D. H. Weiner \& R. L. Morris (Eds.) Research in parapsychology 1987 (pp. 81-85). Scarecrow Press.

Berger, R. E. (1988b). Psi effects without real-time feedback. Journal of Parapsychology, 52, 1-27.

Berger, R. E., \& Honorton, C. (1985). PsiLab II: A standardized psi-testing system. In R. A. White \&J. Solfvin (Eds.) Research in parapsychology 1984 (pp. 68-71). Scarecrow Press.

Berger, R. E., Schechter, E. I., \& Honorton, C. (1986). A preliminary review of performance across three computer psi games. In D. H. Weiner \& D. I. Radin (Eds.) Research in parapsychology 1985 (pp. 1-3). Scarecrow Press.

Bierman, D. J. (1985). A retro and direct PK test for babies with the manipulation of feedback: A first trial of independent replication using software exchange. European Journal of Parapsychology, 5, 373-390.

Bierman, D. J. (1996). Exploring correlations between local emotional and global emotional events and the behavior of a random number generator. Journal of Scientific Exploration, 10, 363-373.

Bierman, D. J. (1998). Do psi phenomena suggest radical dualism? In S. R. Hameroff, A. W. Kazniak, \& A. C. Scott (Eds.) Toward a science of consciousness II: The second TUSCON discussions and debates (pp. 709-713). Bradford/MIT Press.

Bierman, D. J. (2001). On the nature of anomalous phenomena: Another reality between the world of subjective consciousness and the objective world of physics? In P. van Loocke (Ed.) The physical nature of consciousness (pp. 269292). Benjamins Publishing.

Bierman, D. J., \& Houtkooper, J. M. (1975). Exploratory PK tests with a high speed programmable random number generator. European Journal of Parapsychology, 1(1), 1-14.

Bierman, D. J., \& Jolij, J. J. (2020). Dealing with the experimenter effect. Journal of Scientific Exploration, 34, 703-709.

Bierman, D. J., \& van Gelderen, W. J. M. (1994). Geomagnetic activity and PK on a low and high trial-rate RNG. Proceedings of Presented Papers: The Parapsychological Association 37th Annual Convention (pp. 50-56). Parapsychological Association.

Biondi, M. (2009). Defining the picture: A pragmatic approach to the nonrecurrent spontaneous psychokinesis cases. Proceedings of the 8th European SSE Meeting: "Frontiers of Biology and Contemporary Physics" (pp. 57-63). Society for Scientific Exploration.

Blasband, R. A. (2000). The ordering of random events by emotional expression. Journal of Scientific Exploration, 14, 195-216.

Bösch, H., Steinkamp, F., \& Boller, E. (2006a). Examining psychokinesis: The interaction of human intention with random number generators-A meta- 
analysis. Psychological Bulletin, 132, 497-523.

Bösch, H., Steinkamp, F., \& Boller, E. (2006b). In the eye of the beholder: Reply to Wilson and Shadish (2006) and Radin, Nelson, Dobyns, and Houtkooper (2006). Psychological Bulletin, 132, 533-537.

Bradish, G. J., Dobyns, Y. H., Dunne, B. J., Jahn, R. G., Nelson, R. D., Haaland, J. E., \& Hamer, S. M. (1998). Apparatus and method for distinguishing events which collectively exceed chance expectations and thereby controlling an output. U.S. Patent No. 5,830,064. U.S. Patent and Trademark Office.

Braud, W. G. (1978). Recent investigations of microdynamic psychokinesis, with special emphasis on the roles of feedback, effort, and awareness. European Journal of Parapsychology, 2, 137-162.

Braud, W. G. (1981). A long-distance, time-displaced psychokinesis experiment with selected subjects: A preliminary experiment. In W. G. Roll \& J. Beloff (Eds.) Research in parapsychology 1980 (pp. 98-100). Scarecrow Press.

Braud, W. G. (1990). Meditation and psychokinesis. Parapsychology Review, 21(1), 9-11.

Braud, W. G. (2003). Distant mental influence: Its contributions to science, healing, and human interactions. Hampton Roads.

Braud, W. G., Smith, G., Andrew, K., \& Willis, S. (1976). Psychokinetic influences on random number generators during evocation of "analytic" vs. "nonanalytic" modes of information processing. In J. D. Morris, W. G. Roll, \& R. L. Morris (Eds.) Research in parapsychology 1975 (pp. 85-88). Scarecrow Press.

Braude, S. E. (1991). The limits of influence: Psychokinesis and the philosophy of science. Routledge.

Brookes-Smith, C. (1973). Data-tape recorded experimental PK phenomena. Journal of the Society for Psychical Research, 47, 69-89.

Brookes-Smith, C., \& Hunt, D. W. (1970). Some experiments in psychokinesis. Journal of the Society for Psychical Research, 45, 265-281.

Broughton, R. S. (1979). An experiment with the head of Jut. European Journal of Parapsychology, 2, 337-357.

Broughton, R. S. (1983). Brain hemisphere specialization and ESP: What have we learned? Proceedings of Presented Papers: The Parapsychological Association 26th Annual Convention (pp. 373-384). Parapsychological Association.

Broughton, R. S. (1999). Exploring repeated sampling techniques in field-RNG research. Proceedings of Presented Papers: The Parapsychological Association 42nd Annual Convention (pp. 35-47). Parapsychological Association.

Broughton, R. S., \& Higgins, C. A. (1994). An investigation of micro-PK and geomagnetism. Proceedings of Presented Papers: The Parapsychological Association 37th Annual Convention (pp. 87-94). Parapsychological Association.

Broughton, R. S., \& Perlstrom, J. R. (1985). Results of a special subject in a computerized PK game. In R. White \& J. Solfvin (Eds.) Research in parapsychology 1984 (pp. 78-81). Scarecrow Press. 
Broughton, R. S., \& Perlstrom, J. R. (1986). PK experiments with a competitive computer game. Journal of Parapsychology, 50, 193-211.

Broughton, R. S., \& Perlstrom, J. R. (1992). PK in a competitive computer game: A replication. Journal of Parapsychology, 56, 291-305.

Broughton, R. S., Millar, B., Beloff, J., \& Wilson, K. (1978). A PK investigation of the experimenter effect and its psi-based component. In W. G. Roll (Ed.) Research in parapsychology 1977 (pp. 41-48). Scarecrow Press.

Campbell, W. H. (1997). Introduction to geomagnetic fields. Cambridge University Press.

Carrington, H. (1920). The physical phenomena of spiritualism (3rd Ed.). American Universities Publishing.

Carrington, H., \& Fodor, N. (1951). Haunted people: Story of the poltergeist down the centuries. Signet Books/New American Library.

Caswell, J. M., Juden-Kelly, L., Vares, D. A. E., \& Persinger, M. A. (2014). An investigation of solar features, test environment, and gender related to consciousness-correlated deviations in a random physical system. Journal of Scientific Exploration, 28, 453-476.

Close, J. (2012). Are stress responses to geomagnetic storms mediated by the cryptochrome compass system? Proceedings of the Royal Society B, 279, 2081-2090.

Cohen, R. J., Swerdlik, M. E., \& Phillips, S. M. (1996). Psychological testing and assessment: An introduction to tests and measurement (3rd Ed.). Mayfield Publishing.

Collesso, T., Forrester, M., \& Barušs, I. (2021). The effects of meditation and visualization on the direct mental influence of random event generators. Journal of Scientific Exploration, 35, 311-344. https://dx.doi. org/10.31275/20211891

Cornell, T. (2002). Investigating the paranormal. Helix Press.

Crawford, C. C., Jonas, W. B., Nelson, R., Wirkus, M., \& Wirkus, M. (2003). Alterations in random event measures associated with a healing practice. Journal of Alternative and Complementary Medicine, 9, 345-353.

Crookes, W. (1889). Notes of séances with D. D. Home. Proceedings of the Society for Psychical Research, 6, 98-127.

Davidson, J. P., \& Duffin, C. J. (2012). Stones and spirits. Folklore, 123, 99-109. https://dx.doi.org/10.1080/0015587X.2012.643012

Davis, J. W., \& Morrison, M. D. (1978). A test of the Schmidt model's prediction concerning multiple feedback in a PK task. In W. G. Roll (Ed.) Research in parapsychology 1977 (pp. 163-168). Scarecrow Press.

Debes, J., \& Morris, R. (1982). Comparison of striving and nonstriving instructional sets in a PK study. Journal of Parapsychology, 46, 297-312.

Delanoy, D. L. (2001). Anomalous psychophysiological responses to remote cognition: The DMILS studies. European Journal of Parapsychology, 16, 29-40. 
Dobyns, Y. H. (2000). Overview of several theoretical models on PEAR data. Journal of Scientific Exploration, 14, 163-194.

Dobyns, Y. H. (2003). Comments on Freedman, Jeffers, Saeger, Binns, and Black: "Effects of frontal lobe lesions on intentionality and random physical phenomena." Journal of Scientific Exploration, 17, 669-685.

Dobyns, Y. H. (2006). Retrocausal information flow: What are the consequences of knowing the future? In D. P. Sheehan (Ed.) Frontiers of time, retrocausationExperiment and theory (pp. 273-292). AIP Conference Proceedings Vol. 863. American Institute of Physics.

Dobyns, Y. (2015). The PEAR laboratory: Explorations and observations. In D. Broderick \& B. Goertzel (Eds.) Evidence for psi: Thirteen empirical research reports (pp. 213-236). McFarland.

Dobyns, Y. H., \& Nelson, R. D. (1998). Empirical evidence against decision augmentation theory. Journal of Scientific Exploration, 12, 231-257.

Dobyns, Y. H., Dunne, B. J., Jahn, R. G., \& Nelson, R. D. (2004). The MegaREG experiment: Replication and interpretation. Journal of Scientific Exploration, 18, 369-397.

Dobyns, Y. H., Valentino, J. C., Dunne, B. J., \& Jahn, R. G. (2007). The Yantra experiment. Journal of Scientific Exploration, 21, 261-279.

Dunne, B. J. (1991). Co-operator experiments with an REG device. Technical Note PEAR 91005. Princeton Engineering Anomalies Research, School of Engineering \& Applied Science, Princeton University.

Dunne, B. J. (1998). Gender differences in human/machine anomalies. Journal of Scientific Exploration, 12, 3-55.

Dunne, B. J., \& Jahn, R. G. (1992). Experiments in remote human/machine interaction. Journal of Scientific Exploration, 6, 311-332.

Dunne, B. J., \& Jahn, R. G. (1995). Consciousness and anomalous physical phenomena. Technical Note PEAR 95004. Princeton Engineering Anomalies Research, School of Engineering \& Applied Science, Princeton University.

Dunne, B. J., Dobyns, Y. H., Jahn, R. G., \& Nelson, R. D. (1994). Series position effects in random event generator experiments. Journal of Scientific Exploration, 8, 197-215.

Eccles, J. (1977). The human person in its two-way relationship to the brain. In J. D. Morris, W. G. Roll, \& R. L. Morris (Eds.) Research in parapsychology 1976 (pp. 251-262). Scarecrow Press.

Eccles, J. (1986). Do mental events cause neural events analogously to the probability fields of quantum mechanics? Proceedings of the Royal Society of London B, 227, 411-428.

Feather, S. R., \& Rhine, L. E. (1969). PK experiments with same and different targets. Journal of Parapsychology, 33, 213-227.

Feather, S. R., \& Rhine, L. E. (1971). A help-hinder comparison. In J. B. Rhine (Ed.) Progress in parapsychology (pp. 86-96). Parapsychology Press. 
Flammarion, C. (1924). Haunted houses. D. Appleton.

Freedman, M. (2010). Psi and the brain. In S. Krippner \& H. L. Friedman (Eds.) Mysterious minds: The neurobiology of psychics, mediums, and other extraordinary people (pp. 151-161). Praeger/ABC-CLIO.

Freedman, M., Binns, M., Gao, F., Holmes, M., Roseborough, A., Strother, S., Vallesi, A., Jeffers, S., Alain, C., Whitehouse, P., Ryan, J. D., Chen, R., Cusimano, M. D., \& Black, S. E. (2018). Mind-matter interactions and the frontal lobes of the brain: A novel neurobiological model of psi inhibition. Explore: The Journal of Science \& Healing, 14, 76-85. https://dx.doi. org/10.1016/j.explore.2017.08.003

Freedman, M., Jeffers, S., Saeger, K., Binns, M., \& Black, S. (2003). Effects of frontal lobe lesions on intentionality and random physical phenomena. Journal of Scientific Exploration, 17, 651-668.

Gauld, A., \& Cornell, A. D. (1979). Poltergeists. Routledge \& Kegan Paul.

Giroldini, W. (1991). Eccles' model of mind-brain interaction and psychokinesis: A preliminary study. Journal of Scientific Exploration, 5, 145-161.

Giroldini, W., \& Pederzoli, L. (2021). EEG activity during mental influence on a random signal generator. Journal of Scientific Exploration, 35, 267-286. https://dx.doi.org/10.31275/20211909

Gissurarson, L. R. (1986). RNG-PK microcomputer "games" overviewed: An experiment with the videogame "Psi Invaders." European Journal of Parapsychology, 6, 199-215.

Gissurarson, L. R. (1990-1991). Some PK attitudes as determinants of PK performance. European Journal of Parapsychology, 8, 112-122.

Gissurarson, L. R. (1992a). Studies of methods of enhancing and potentially training psychokinesis: A review. Journal of the American Society for Psychical Research, 86, 303-346.

Gissurarson, L. R. (1992b). The psychokinesis effect: Geomagnetic influence, age and sex differences. Journal of Scientific Exploration, 6, 157-165.

Gissurarson, L. R. (1997). Descriptive analysis of mentations on volitional tasks. Journal of the Society for Psychical Research, 62, 22-35.

Gissurarson, L. R., \& Morris, R. L. (1991). Examination of six questionnaires as predictors of psychokinesis performance. Journal of Parapsychology, 55, 119-141.

Gregory, A. (1985). The strange case of Rudi Schneider. Scarecrow Press.

Grote, H. (2017). Multiple-analysis correlation study between human psychological variables and binary random events. Journal of Scientific Exploration, 31, 231254.

Grote, H. (2021). Mind-matter entanglement correlations: Blind analysis of a new correlation matrix experiment. Journal of Scientific Exploration, 35, 287-310. https://dx.doi.org/10.31275/20211931

Hansel, C. E. M. (1980). ESP and parapsychology: A critical re-evaluation. Prometheus. 
Hansel, C. E. M. (1981, Spring). A critical analysis of H. Schmidt's psychokinesis experiments. The Skeptical Inquirer, 26-33.

Hansen, G. P. (1990). A cooperation-competition PK experiment with computerized horse races. Journal of Parapsychology, 54, 21-33.

Hansen, G. P. (2006). Correspondence. Journal of the Society for Psychical Research, 70, 254 .

Heath, P. R. (2000). The PK zone: A phenomenological study. Journal of Parapsychology, 64, 53-72.

Heath, P. R. (2011). Mind-matter interaction: Historical reports, research and firsthand accounts. McFarland.

Helfrich, W. (2007). Is the psychokinetic effect as found with binary random number generators suitable to account for mind-brain interaction? Journal of Scientific Exploration, 21, 689-705.

Heseltine, G. L. (1977). Electronic random number generator operation associated with EEG activity. Journal of Parapsychology, 41, 103-118.

Heseltine, G. L., \& Mayer-Oakes, S. A. (1978). Electronic random generator operation and EEG activity: Further studies. Journal of Parapsychology, 42, $123-136$.

Honorton, C. (1977). Psi and internal attention states. In B. B. Wolman (Ed.) Handbook of parapsychology (pp. 435-472). Van Nostrand Reinhold.

Honorton, C., \& Barksdale, W. (1972). PK performance with waking suggestions for muscle tension versus relaxation. Journal of the American Society for Psychical Research, 66, 208-214.

Honorton, C., \& Tremmel, L. (1979). Psi correlates of volition: A preliminary test of Eccles' "neurophysiological hypothesis" of mind-brain interaction. In W. G. Roll (Ed.) Research in parapsychology 1978 (pp. 36-38). Scarecrow Press.

Honorton, C., Barker, P., \& Sondow, N. (1983). Feedback and participant-selection parameters in a computer RNG study. In W. G. Roll, J. Beloff, \& R. A. White (Eds.) Research in parapsychology 1982 (pp. 157-159). Scarecrow Press.

Houtkooper, J. M. (1977). A study of repeated retroactive psychokinesis in relation to direct and random PK effects. European Journal of Parapsychology, 1(4), $1-20$.

Houtkooper, J. M. (2002a). Arguing for an observational theory of paranormal phenomena. Journal of Scientific Exploration, 16, 171-185.

Houtkooper, J. M. (2002b). A pilot experiment with evoked psychokinetic responses: Circumventing cognitive interference? Proceedings of Presented Papers: The Parapsychological Association 45th Annual Convention (pp. 104115). Parapsychological Association.

Houtkooper, J. M. (2004). Exploring volitional strategies in the mind-machine interaction replication. Proceedings of Presented Papers: The Parapsychological Association 47th Annual Convention (pp. 51-64). Parapsychological Association. Houtkooper, J. M. (2006a). Experimenter effects and volitional strategies in the 
mind-machine interaction replication. Proceedings of Presented Papers: The Parapsychological Association 49th Annual Convention (pp. 71-84). Parapsychological Association.

Houtkooper, J. M. (2006b). Retrocausation or extant indefinite reality? In D. P. Sheehan (Ed.) Frontiers of time, retrocausation-Experiment and theory (pp. 147-168). AIP Conference Proceedings Vol. 863. American Institute of Physics.

Houtkooper, J. M., Andrews, R., Ganzevles, P. G. J., \& van der Sijde, P. C. (1980). A hierarchical model for the observational theories: A study of subject identity and feedback in repeated retroactive psychokinesis. European Journal of Parapsychology, 3, 221-246.

Hubbard, G. S., \& May, E. C. (1987). Aspects of the measurement and application of geomagnetic indices and extremely low frequency electromagnetic radiation for use in parapsychology. In D. H. Weiner \& R. D. Nelson (Eds.) Research in parapsychology 1986 (pp. 79-82). Scarecrow Press.

Huesmann, M., \& Schriever, F. (1989). Steckbrief des Spuks-Darstellung und Diskussion einer Sammlung von 54 RSPK-Berichten des Freiburger Instituts für Grenzgebiete der Psychologie und Psychohygiene aus den Jahren 1947-1986 [Characteristics of Poltergeists-Presentation and discussion of a collection of 54 RSPK reports from the Freiburg Institute for the Border Regions of Psychology and Mental Health from the years 1947-1986]. Zeitschrift für Parapsychologie und Grenzgebiete der Psychologie, 31, 52-107.

Humphrey, B. M. (1947). Help-hinder comparisons in PK tests. Journal of Parapsychology, 11, 4-13.

Hyman, R. (1981, Spring). Further comments on Schmidt's PK experiments. The Skeptical Inquirer, 34-40.

Ibison, M. (1998). Evidence that anomalous statistical influence depends on the details of the random process. Journal of Scientific Exploration, 12, 407-423.

Ibison, M., \& Jeffers, S. (1998). A double-slit diffraction experiment to investigate claims of consiousness-related anomalies. Journal of Scientific Exploration, 12(4), 543-550.

Irwin, H. J. (1994). The phenomenology of parapsychological experiences. In S. Krippner (Ed.) Advances in parapsychological research 7 (pp. 10-76). McFarland.

Ivtzan, I. (2008). Meditation on consciousness. Journal of Scientific Exploration, 22, 147-159.

Jahn, R. G. (1982). The persistent paradox of psychic phenomena: An engineering perspective. Proceedings of the IEEE, 70, 136-170.

Jahn, R. G., \& Dunne, B. J. (1986). On the quantum mechanics of consciousness, with application to anomalous phenomena. Foundations of Physics, 16, 721772 . 
Jahn, R. G., \& Dunne, B. J. (1987). Margins of reality: The role of consciousness in the physical world. Harcourt Brace Jovanovich.

Jahn, R. G., \& Dunne, B. J. (2005). The PEAR proposition. Journal of Scientific Exploration, 19, 195-245.

Jahn, R. G., \& Dunne, B. J. (2011). Consciousness and the source of reality: The PEAR odyssey. ICRL Press.

Jahn, R., Dunne, B., Bradish, G., Dobyns, Y., Lettieri, A., Nelson, R., Mischo, J., Boller, E., Bösch, H., Vaitl, D., Houtkooper, J., \& Walter, B. (2000). Mind/ Machine Interaction Consortium: PortREG replication experiments. Journal of Scientific Exploration, 14, 499-555.

Jahn, R. G., Dunne, B. J., \& Dobyns, Y. H. (2006). Exploring the possible effects of Johrei techniques on the behavior of random physical systems. Technical Note PEAR 2006.01. Princeton Engineering Anomalies Research, School of Engineering \& Applied Science, Princeton University.

Jahn, R. G., Dunne, B. J., Nelson, R. D., Dobyns, Y. H., \& Bradish, G. J. (1997). Correlations of random binary sequences with pre-stated operator intention: A review of a 12-year program. Journal of Scientific Exploration, 11, 345-367.

Jahn, R. G., Dunne, B. J., Nelson, R. D., Dobyns, Y. H., \& Bradish, G. J. (1999). Response to Jeffers. Journal of Scientific Exploration, 13, 329-331.

Jeffers, S. (1999). Comment on "Correlations of Random Binary Sequences with Pre-Stated Operator Intention: A Review of a 12-Year Program" by R. G. Jahn, B. J. Dunne, R. D. Nelson, Y. H. Dobyns and G. J. Bradish. Journal of Scientific Exploration, 13, 327-329.

Jeffers, S. (2006, May/June). The PEAR proposition: Fact or fallacy? Skeptical Inquirer, 54-57.

Jeffers, S., \& Sloan, J. (1992). A low light level diffraction experiment for anomalies research. Journal of Scientific Exploration, 6(4), 333-352.

Kennedy, J. E., \& Taddonio, J. L. (1976). Experimenter effects in psi research. Journal of Parapsychology, 40, 1-33.

Kolb, B., \& Whishaw, I. Q. (2006). An introduction to brain and behavior (2nd ed.). Worth Publishers.

Krieger, J. (1977). Hemispheric specialization in intentional versus nonintentional psi performance [Abstract]. Journal of Parapsychology, 41, 40-42.

Krippner, S., \& Friedman, H. L. (Eds.) (2010). Mysterious minds: The neurobiology of psychics, mediums, and other extraordinary people. Praeger/ABC-CLIO.

Kugel, W. (2011). A faulty PK meta-analysis. Journal of Scientific Exploration, 25, 4762.

Lawrence, T. (1993). Gathering in the sheep and goats: A meta-analysis of forcedchoice sheep-goat ESP studies, 1947-1993. Proceedings of Presented Papers: The Parapsychological Association 36th Annual Convention (pp. 75-86). Parapsychological Association. 
Levin, J. (2011). Energy healers: Who they are and what they do. Explore: The Journal of Science \& Healing, 7, 13-26. https://dx.doi.org/10.1016/j.explore.2010.10.005 Lowry, R. (1981). Apparent PK effect on computer-generated random digit series. Journal of the American Society for Psychical Research, 75, 209-220.

Lumsden-Cook, J. (2005a). Mind-matter and emotion. Journal of the Society for Psychical Research, 69, 1-17.

Lumsden-Cook, J. (2005b). Affect and random events: Examining the effects of induced emotion upon mind-matter interactions. Journal of the Society for Psychical Research, 69, 128-142.

Lumsden-Cook, J. J., Edwards, S. D., \& Thwala, J. (2006a). An exploratory study into traditional Zulu healing and REG effects. Journal of Parapsychology, 70, 129-138.

Lumsden-Cook, J. J., Thwala, J., \& Edwards, S. D. (2006b). The effects of traditional Zulu healing upon a random event generator. Journal of the Society for Psychical Research, 70, 129-137.

Lyon, J. G. (2000). The solar wind-magnetosphere-ionosphere system. Science, 288, 1987-1991.

Mason, L. I., Patterson, R. P., \& Radin, D. I. (2007). Exploratory study: The random number generator and group meditation. Journal of Scientific Exploration, 21, 295-317.

May, E. C., \& Spottiswoode, S. J. P. (2011a). The Global Consciousness Project: Identifying the source of psi. Journal of Scientific Exploration, 25, 663-682.

May, E. C., \& Spottiswoode, S. J. P. (2011b). The Global Consciousness Project, identifying the source of psi: A response to Nelson and Bancel. Journal of Scientific Exploration, 25, 745-748.

May, E. C., Utts, J. M., \& Spottiswoode, S. J. P. (1995a). Decision augmentation theory: Toward a model of anomalous mental phenomena. Journal of Parapsychology, 59, 195-220.

May, E. C., Utts, J. M., \& Spottiswoode, S. J. P. (1995b). Decision augmentation theory: Applications to the random number generator database. Journal of Scientific Exploration, 9, 453-488.

McConnell, R. A. (1989). Psychokinetic data structure. Journal of the American Society for Psychical Research, 83, 217-226.

Michels, H. (1993). In strijd met het toeval-PK experimenten met en zonder Skepsis [Contrary to chance-PK experiments with and without skeptics]. Skepter, 6(4).

Millar, B. (1978). The observational theories: A primer. European Journal of Parapsychology, 2, 304-332.

Millar, B., \& Mackenzie, P. (1977). A test of intentional versus unintentional PK. In J. D. Morris, W. G. Roll, \& R. L. Morris (Eds.) Research in parapsychology 1976 (pp. 32-35). Scarecrow Press.

Miller, D. I., \& Halpern, D. F. (2014). The new science of cognitive sex differences. 
Trends in Cognitive Sciences, 18, 37-45. https://dx.doi.org/10.1016/j. tics.2013.10.011

Moga, M. (2015, November 20). Random event generator (REG) output during healing touch. "Healing with Intent" blog. https://www.naturalhealingwaves.com/ random-event-generator-reg-output-during-healing-touch/

Morrison, M. D., \& Davis, J. W. (1979). PK with immediate, delayed, and multiple feedback: A test of the Schmidt model's predictions. In W. G. Roll (Ed.) Research in parapsychology 1978 (pp. 117-120). Scarecrow Press.

Nelson, R. D. (1999). The physical basis of intentional healing systems. Technical Report PEAR 99001. Princeton Engineering Anomalies Research, School of Engineering \& Applied Science, Princeton University.

Nelson, R. D. (2001). Correlation of global events with REG data: An Internet-based, nonlocal anomalies experiment. Journal of Parapsychology, 65, 247-271.

Nelson, R. (2008). The emotional nature of global consciousness. Aquém e além do cérebro: 70 simpósio da Fundação Bial [Behind and beyond the brain: Proceedings of the 7th Bial Foundation symposium] (pp. 97-114). Fundação Bial.

Nelson, R. (2011). Reply to May and Spottiswoode on experimenter effect as the explanation for GCP results. Journal of Scientific Exploration, 25, 683-689.

Nelson, R. D. (2015). The Global Consciousness Project: Subtle interconnections and correlations in random data. In D. Broderick \& B. Goertzel (Eds.) Evidence for psi: Thirteen empirical research reports (pp. 237-254). McFarland.

Nelson, R. (2017). Weighting the parameters: A response to Bancel's "Searching for Global Consciousness: A Seventeen Year Exploration." Explore: The Journal of Science \& Healing, 13, 102-105. https://dx.doi.org/10.1016/j. explore.2016.12.002

Nelson, R. D. (2019). Connected: The emergence of global consciousness. ICRL Press.

Nelson, R. D. (2020). The Global Consciousness Project's event-related responses look like brain EEG event-related potentials. Journal of Scientific Exploration, 34, 246-267.

Nelson, R., \& Bancel, P. (2009). To the Editor. Journal of Scientific Exploration, 23, 510-516.

Nelson, R. D., \& Bancel, P. (2011). Effects of mass consciousness: Changes in random data during global events. Explore: The Journal of Science \& Healing, 7, 373-383. https://dx.doi.org/10.1016/j.explore.2011.08.003

Nelson, R. D., \& Dunne, B. J. (1987). Attempted correlation of engineering anomalies with global geomagnetic activity. In D. H. Weiner \& R. D. Nelson (Eds.) Research in parapsychology 1986 (pp. 82-85). Scarecrow Press.

Nelson, R. D., \& Radin, D. I. (2003). FieldREG experiments and group consciousness: Extending REG/RNG research to real-world situations. In W. B. Jonas \& C. C. Crawford (Eds.) Healing, intention, and energy medicine: Science, research methods and clinical implications (pp. 49-57). Churchill Livingstone. 
Nelson, L. A., \& Schwartz, G. E. (2006). Consciousness and the anomalous organization of random events: The role of absorption. Journal of Scientific Exploration, 20, 523-544.

Nelson, R. D., Bradish, G. J., \& Dobyns, Y. H. (1989). Random event generator qualification, calibration, and analysis. Technical Note PEAR 89001. Princeton Engineering Anomalies Research, School of Engineering \& Applied Science, Princeton University.

Nelson, R. D., Bradish, G. J., Dobyns, Y. H., Dunne, B. J., \& Jahn, R. G. (1996). FieldREG anomalies in group situations. Journal of Scientific Exploration, 10, $111-141$.

Nelson, R. D., Dunne, B. J., \& Jahn, R. G. (1982). Psychokinesis studies with a Fabry-Perot interferometer. In W. G. Roll, R. L. Morris, \& R. A. White (Eds.) Research in parapsychology 1981 (pp. 47-50). Scarecrow Press.

Nelson, R. D., Jahn, R. G., Dobyns, Y. H., \& Dunne, B. J. (2000). Contributions to variance in REG experiments: ANOVA models and specialized subsidiary analyses. Journal of Scientific Exploration, 14, 73-89.

Nelson, R. D., Jahn, R. G., \& Dunne, B. J. (1986). Operator-related anomalies in physical systems and information processes. Journal of the Society for Psychical Research, 53, 261-285.

Nelson, R. D., Jahn, R. G., Dunne, B. J., Dobyns, Y. H., \& Bradish, G. J. (1998). FieldREG II: Consciousness field effects: Replications and explorations. Journal of Scientific Exploration, 12, 425-454.

Nisbet, B. (1973). Table turning: A brief historical note mainly of the period 18481853. Journal of the Society for Psychical Research, 47, 96-106.

Owen, I. M., \& Sparrow, M. (1976). Conjuring up Philip: An adventure in psychokinesis. Harper \& Row.

Pallikari, F. (2015). Investigating the nature of intangible brain-machine interaction. Journal of Social Sciences and Humanities, 1, 499-508.

Pallikari, F. (2016). The balancing effect in brain-machine interaction. https://arxiv.org/ abs/1602.00808

Pallikari-Viras, F. (1997). Further evidence for a statistical balancing in probabilistic systems influenced by the anomalous effect of conscious intention. Journal of the Society for Psychical Research, 62, 114-137.

Pallikari-Viras, F. (1998). On the balancing effect hypothesis. In N. L. Zingrone, M. J. Schlitz, C. S. Alvarado, \& J. Milton (Eds.) Research in parapsychology 1993 (pp. 102-103). Scarecrow Press.

Palmer, J. (1997). The challenge of experimenter psi. European Journal of Parapsychology, 13, 110-125.

Palmer, J., \& Kramer, W. (1984). Internal state and temporal factors in psychokinesis. Journal of Parapsychology, 48, 1-25.

Palmer, J., \& Kramer, W. (1987). Release of effort in RNG PK: An attempted replication and extension. Journal of Parapsychology, 51, 125-136. 
Palmer, J., \& Millar, B. (2015). Experimenter effects in parapsychology research. In E. Cardeña, J. Palmer, \& D. Marcusson-Clavertz (Eds.) Parapsychology: A handbook for the 21st century (pp. 293-298). McFarland.

Palmer, J., \& Perlstrom, J. (1987). Random event generator PK in relation to task instructions. In D. H. Weiner \& R. D. Nelson (Eds.) Research in parapsychology 1986 (pp. 17-20). Scarecrow Press.

Palmer, S. J., Rycroft, M. J., \& Cermack, M. (2006). Solar and geomagnetic activity, extremely low frequency magnetic and electric fields and human health at the Earth's surface. Surveys in Geophysics, 27, 557-595. https://dx.doi. org/10.1007/s10712-006-9010-7

Parker, A., \& Millar, B. (2014). Revealing psi secrets: Successful experimenters seem to succeed by using their own psi. Journal of Parapsychology, 78, 39-55.

Pilkington, R. (2006). The spirit of Dr. Bindelof: The enigma of séance phenomena. Anomalist Books.

Pratt, J. G. (1949). The meaning of performance curves in ESP and PK test data. Journal of Parapsychology, 13, 9-23.

Pratt, J. G. (1960). The case for psychokinesis. Journal of Parapsychology, 24, 171-188.

PRL (Psychophysical Research Laboratories). (1985). PsiLab II. The Paranormal Trickster Blog. http://paranormaltrickster.blogspot.com/2009/12/ psychophysical-research-laboratories.html

Puhle, A. (1999). Six selected poltergeist cases from the 1700 in Germany. Proceedings of Presented Papers: The Parapsychological Association 42nd Annual Convention (pp. 278-288). Parapsychological Association.

Radin, D. I. (1982a). Mental influence on machine-generated random events: Six experiments. In W. G. Roll, R. L. Morris, \& R. A. White (Eds.) Research in parapsychology 1981 (pp. 141-142). Scarecrow Press.

Radin, D. I. (1982b). Experimental attempts to influence pseudorandom number sequences. Journal of the American Society for Psychical Research, 76, 359-374.

Radin, D. I. (1985). Pseudorandom number generators in psi research. Journal of Parapsychology, 49, 303-328.

Radin, D. I. (1989). Searching for "signatures" in anomalous human-machine interaction data: A neural network approach. Journal of Scientific Exploration, $3,185-200$.

Radin, D. I. (1993a). Neural network analyses of consciousness-related patterns in random sequences. Journal of Scientific Exploration, 7, 355-373.

Radin, D. I. (1993b). Environmental modulation and statistical equilibrium in mind-matter interaction. Subtle Energies, 4, 1-30.

Radin, D. I. (1996). Towards a complex systems model of psi performance. Subtle Energies \& Energy Medicine, 7, 35-69.

Radin, D. I. (1997). The conscious universe: The scientific truth of psychic phenomena. HarperEdge.

Radin, D. (2006). Entangled minds: Extrasensory experiences in a quantum reality. 
Paraview Pocket Books.

Radin, D. I., \& Ferrari, D. C. (1991). Effects of consciousness on the fall of dice: A meta-analysis. Journal of Scientific Exploration, 5, 61-83.

Radin, D. I., \& Nelson, R. D. (1989). Evidence for consciousness-related anomalies in random physical systems. Foundations of Physics, 19, 1499-1514.

Radin, D. I., \& Nelson, R. D. (2003). A meta-analysis of mind-matter interaction experiments from 1959 to 2000. In W. B. Jonas \& C. C. Crawford (Eds.) Healing, intention, and energy medicine: Science, research methods and clinical implications (pp. 39-48). Churchill Livingstone.

Radin, D. I., \& Utts, J. (1989). Experiments investigating the influence of intention on random and pseudorandom events. Journal of Scientific Exploration, 3, 65-79.

Radin, D. I., May, E. C., \& Thomson, M. J. (1986). Psi experiments with random number generators: Meta-analysis Part I. In D. H. Weiner \& D. I. Radin (Eds.) Research in parapsychology 1985 (pp. 14-17). Scarecrow Press.

Radin, D. I., Nelson, R., Dobyns, Y., \& Houtkooper, J. (2006a). Reexamining psychokinesis: Comment on Bösch, Steinkamp, and Boller (2006). Psychological Bulletin, 132, 529-532.

Radin, D. I., Nelson, R., Dobyns, Y., \& Houtkooper, J. (2006b). Assessing the evidence for mind-matter interaction effects. Journal of Scientific Exploration, 20, 361-374.

Radin, D. I., Rebman, J. M., \& Cross, M. P. (1996). Anomalous organization of random events by group consciousness: Two exploratory experiments. Journal of Scientific Exploration, 10, 143-168.

Radin, D. I., Taft, R., \& Yount, G. (2004). Effects of healing intention on cultured cells and truly random events. Journal of Alternative and Complementary Medicine, 10, 103-112.

Reed, S. K. (2004). Cognition: Theory and applications (6th Ed.). Thomson/ Wordsworth.

Reeves, M. P., \& Rhine, J. B. (1943). The PK effect: II. A study in declines. Journal of Parapsychology, 7, 76-93.

Rhine, J. B. (1969). Position effects in psi test results. Journal of Parapsychology, 33, 136-157.

Rhine, L. E. (1963). Spontaneous physical effects and the psi process. Journal of Parapsychology, 27, 84-122.

Rhine, L. E. (1970). Mind over matter: Psychokinesis. Macmillan Publishing.

Roe, C. A., Davey, R., \& Stevens, P. (2004). Arousal and performance at ESP and PK tasks using a common protocol. European Journal of Parapsychology, 19, 29-43.

Roe, C. A., Sonnex, C., \& Roxburgh, E. C. (2015). Two meta-analyses of noncontact healing studies. Explore: The Journal of Science \& Healing, 11, 11-23. https:// dx.doi.org/10.1016/j.explore.2014.10.001 
Rogo, D. S. (1986). On the track of the poltergeist. Prentice-Hall.

Roll, W. G. (1977). Poltergeists. In B. B. Wolman (Ed.) Handbook of parapsychology (pp. 382-413). Van Nostrand Reinhold.

Roll, W. G. (1983). Recurrent and nonrecurrent psi effects. Journal of Parapsychology, 47, 341-346.

Rosenthal, R. \& Rubin, D. B. (1989). Effect size estimation for one-sample multiplechoice-type data: Design, analysis, and meta-analysis. Psychological Bulletin, 106, 332-337.

Rosenthal, R., \& Rubin, D. B. (1992-1993). An effect size estimator for parapsychological research. European Journal of Parapsychology, 9, 1-11.

Rowe, W. D. (1998). Physical measurement of episodes of focused group energy. Journal of Scientific Exploration, 12, 569-581.

Ryan, A., \& Spottiswoode, S. J. P. (2015). Variation of ESP by season, local sidereal time, and geomagnetic activity. In E. C. May \& S. B. Marwaha (Eds.) Extrasensory perception: Support, skepticism, and science (Vol. 1), (pp. 377-394). Praeger/ABC-CLIO.

Schechter, E. I., Barker, P., \& Varvoglis, M. P. (1983). A preliminary study with a PK game involving distraction from the psi task. In W. G. Roll, J. Beloff, \& R. A. White (Eds.) Research in parapsychology 1982 (pp. 152-154). Scarecrow Press.

Schechter, E. I., Barker, P., \& Varvoglis, M. P. (1984). A second study with the "Psi Ball” RNG-PK game. In R. A. White \& R. S. Broughton (Eds.) Research in parapsychology 1983 (pp. 93-94). Scarecrow Press.

Schechter, E. I., Honorton, C., Barker, P., \& Varvoglis, M. P. (1984). Relationship between participant traits and scores on two computer-controlled RNG-PK games. In R. A. White \& R. S. Broughton (Eds.) Research in parapsychology 1983 (pp. 32-33). Scarecrow Press.

Schlitz, M., \& Braud, W. (1997). Distant intentionality and healing: Assessing the evidence. Alternative Therapies in Health and Medicine, 3, 62-73.

Schmeidler, G. R. (1994). ESP experiments 1978-1992: The glass is half full. In S. Krippner (Ed.) Advances in parapsychological research 7 (pp. 104-197). McFarland.

Schmeidler, G. R., \& McConnell, R. A. (1958). ESP and personality patterns. Yale University Press.

Schmidt, H. (1970a). A PK test with electronic equipment. Journal of Parapsychology, $34,175-181$.

Schmidt, H. (1970b). A quantum mechanical random number generator for psi tests. Journal of Parapsychology, 34, 219-224.

Schmidt, H. (1970c). Quantum-mechanical random number generator. Journal of Applied Physics, 41, 462-468.

Schmidt, H. (1971a). Mental influence on random events. New Scientist and Science Journal, 50, 757-758.

Schmidt, H. (1971b). A quantum process in psi testing. In J. B. Rhine (Ed.) Progress 
in parapsychology (pp. 28-35). Parapsychology Press.

Schmidt, H. (1973). PK tests with a high-speed random number generator. Journal of Parapsychology, 37, 105-118.

Schmidt, H. (1974a). Psychokinesis. In E. D. Mitchell \& J. White (Eds.) Psychic exploration: A challenge for science (pp. 179-193). G. P. Putnam's Sons.

Schmidt, H. (1974b). Comparison of PK action on two different random number generators. Journal of Parapsychology, 38, 47-55.

Schmidt, H. (1975). Toward a mathematical theory of psi. Journal of the American Society for Psychical Research, 69, 301-319.

Schmidt, H. (1976). PK effect on pre-recorded targets. Journal of the American Society for Psychical Research, 70, 267-291.

Schmidt, H. (1977). A simple random number generator for use with minicomputers. Journal of the American Society for Psychical Research, 71, 171-176.

Schmidt, H. (1978). A take-home test in PK using pre-recorded targets. In W. G. Roll (Ed.) Research in parapsychology 1977 (pp. 31-36). Scarecrow Press.

Schmidt, H. (1979). Use of stroboscopic light as rewarding feedback in a PK test with prerecorded and momentarily-generated random events. In W. G. Roll (Ed.) Research in parapsychology 1978 (pp. 115-117). Scarecrow Press.

Schmidt, H. (1981). PK tests with pre-recorded and pre-inspected seed numbers. Journal of Parapsychology, 45, 87-98.

Schmidt, H. (1982). Collapse of the state vector and psychokinetic effect. Foundations of Physics, 12, 565-581.

Schmidt, H. (1985). Addition effect for PK with prerecorded targets. Journal of Parapsychology, 49, 229-244.

Schmidt, H. (1987a). The strange properties of psychokinesis. Journal of Scientific Exploration, 1, 103-118.

Schmidt, H. (1987b). Alcock's critique of Schmidt's experiments. Behavioral and Brain Sciences, 10, 609.

Schmidt, H. (1990). Correlation between mental processes and external random events. Journal of Scientific Exploration, 4, 233-241.

Schmidt, H. (1993a). Observation of a psychokinetic effect under highly controlled conditions. Journal of Parapsychology, 57, 351-372.

Schmidt, H. (1993b). Non-causality as the earmark of psi. Journal of Scientific Exploration, 7, 125-132.

Schmidt, H. (1997). Random generators and living systems as targets in retro-PK experiments. Journal of the American Society for Psychical Research, 91, 1-13.

Schmidt, H. (2000). PK tests in a pre-sleep state. Journal of Parapsychology, 64, 317-331.

Schmidt, H. (2009). A puzzling aspect of the "Global Consciousness Project." Journal of Scientific Exploration, 23, 507-510.

Schmidt, H., \& Braud, W. (1993). New PK tests with an independent observer. Journal of Parapsychology, 57, 227-239. 
Schmidt, H., \& Dalton, K. (1999). PK tests with repeated efforts on prerecorded events. Journal of Parapsychology, 63, 275-283.

Schmidt, H., \& Schlitz, M. J. (1989). A large-scale pilot PK experiment with prerecorded random events. In L. A. Henkel \& R. E. Berger (Eds.) Research in parapsychology 1988 (pp. 6-10). Scarecrow Press.

Schmidt, H., \& Stapp, H. (1993). PK with prerecorded random events and the effects of preobservation. Journal of Parapsychology, 57, 331-349.

Schmidt, H., \& Terry, J. C. (1977). Search for a relationship between brainwaves and PK performance. In J. D. Morris, W. G. Roll, \& R. L. Morris (Eds.) Research in parapsychology 1976 (pp. 30-32). Scarecrow Press.

Schmidt, H., Morris, R., \& Hardin, C. L. (1994). Channeling evidence for psychokinetic effect to independent observers: A failure to replicate. In E. W. Cook \& D. L. Delanoy (Eds.) Research in parapsychology 1991 (pp. 34-38). Scarecrow Press.

Schmidt, H., Morris, R., \& Rudolph, L. (1986). Channeling evidence for a PK effect to independent observers. Journal of Parapsychology, 50, 1-15.

Schmidt, S. (2008). Aristotle's fly-Remarks on anomaly research and a review on the relationship between meditation and psi. Aquém e além do cérebro: 70 simpósio da Fundação Bial [Behind and beyond the brain: Proceedings of the 7 th Bial Foundation symposium] (pp.73-95). Fundação Bial.

Schmidt, S. (2012). Can we help just by good intentions? A meta-analysis of experiments on distant intention effects. Journal of Alternative and Complementary Medicine, 18, 529-533. https://dx.doi.org/10.1089/ acm.2011.0321

Schmidt, S., Schneider, R., Utts, J., \& Walach, H. (2004). Distant intentionality and the feeling of being stared at: Two meta-analyses. British Journal of Psychology, 95, 235-247.

Schub, M. H. (2006). A critique of the parapsychological random number generator meta-analyses of Radin and Nelson. Journal of Scientific Exploration, 20, 402-419.

Schwartz, G. E. R., Russek, L. G. S., She, Z.-S., Song, L. Y. X., \& Xin, Y. (1997). Anomalous organization of random events during an international qigong meeting: Evidence for group consciousness or accumulated qi fields? Subtle Energies \& Energy Medicine, 8, 55-65.

Shimizu, T., \& Ishikawa, M. (2012). Field RNG experiments using short movies: An examination of the focused-attention and emotion hypotheses. Journal of International Society of Life Information Science, 30, 17-24.

Springer, S. P., \& Deutsch, G. (1998). Left brain, right brain: Perspectives from cognitive neuroscience (5th ed.). W. H. Freeman \& Company.

Stapp, H. P. (1994). Theoretical model of a purported empirical violation of the predictions of quantum theory. Physical Review A, 50, 18-22.

Stanford, R. G., \& Fox, C. (1975). An effect of release of effort in a psychokinetic task. 
In J. D. Morris, W. G. Roll, \& R. L. Morris (Eds.) Research in parapsychology 1974 (pp. 61-63). Scarecrow Press.

Stevens, P. (2005). The effect of weak magnetic fields on a random event generator: Reconsidering the role of geomagnetic fluctuations in microPK studies. European Journal of Parapsychology, 20, 135-149.

Storm, L., \& Tressoldi, P. E. (2017). Gathering in more sheep and goats: A metaanalysis of forced-choice sheep-goat ESP studies, 1994-2015. Journal of the Society for Psychical Research, 81, 79-107.

Talbert, R., \& Debes, J. (1982). Time-displacement psychokinetic effects on a random-number generator using varying amounts of feedback. In W. G. Roll, R. L. Morris, \& R. A. White (Eds.) Research in parapsychology 1981 (pp. 58-61). Scarecrow Press.

Taylor, R. (1996). Training imagery skills for enhanced psi functioning: Two experiments with athletes. European Journal of Parapsychology, 12, 1-22.

Terry, J., \& Schmidt, H. (1978). Conscious and subconscious PK tests with prerecorded targets. In W. G. Roll (Ed.) Research in parapsychology 1977 (pp. 36-41). Scarecrow Press.

Thalbourne, M. A. (2006). Kundalini and the output of a random number generator. Journal of Parapsychology, 70, 303-333.

Thalbourne, M. A. (2008). A second study on the effect of Kundalini on the output of a random number generator. Journal of Parapsychology, 72, 155-168.

Thalbourne, M. A., \& Storm, L. (2015). Debunking an alleged feedback artifact in Thalbourne's RNG studies. Australian Journal of Parapsychology, 15, 81-87.

Thompson, A. (1994). Serial position effects in the psychological literature. Journal of Scientific Exploration, 8, 211-215.

Tremmel, L., \& Honorton, C. (1980). Directional PK effects with a computer-based random generator system: A preliminary study. In W. G. Roll (Ed.) Research in parapsychology 1979 (pp. 69-71). Scarecrow Press.

Tressoldi, P. E. (2012). Replication unreliability in psychology: Elusive phenomena or "elusive" statistical power? Frontiers in Psychology, 3, 218. https://dx.doi. org/10.3389/fpsyg.2012.00218

Tressoldi, P., Pederzoli, L., Caini, P., Ferrini, A., Melloni, S., Richeldi, D., Richeldi, F., \& Dum, G. D. (2014). Mind-matter interaction at a distance of $190 \mathrm{~km}$ : Effects on a random event generator using a cutoff method. NeuroQuantology, 12, 337-343.

Tressoldi, P., Pederzoli, L., Prati, E., \& Semenzato, L. (2020). Mind control of a distant electronic device: A proof-of-concept pre-registered study. Journal of Scientific Exploration, 34, 233-245.

Ullman, M. (2001). The Bindelof sitter group and its influence on the lives of the participants. Journal of the American Society for Psychical Research, 95, 13-109. Varvoglis, M. P. (1989). A "psychic contest" using a computer-RNG task in a nonlaboratory setting. In L. A. Henkel \& R. E. Berger (Eds.) Research in 
parapsychology 1988 (pp. 10-15). Scarecrow Press.

Varvoglis, M. P., \& Bancel, P. (2015). Micro-psychokinesis. In E. Cardeña, J. Palmer, \& D. Marcusson-Clavertz (Eds.) Parapsychology: A handbook for the 21st century (pp. 266-281). McFarland.

Varvoglis, M. P., \& Bancel, P. A. (2016). Micro-psychokinesis: Exceptional or universal? Journal of Parapsychology, 80, 37-44.

Varvoglis, M. P., \& McCarthy, D. (1986). Conscious-purposive focus and PK: RNG activity in relation to awareness, task-orientation, and feedback. Journal of the American Society for Psychical Research, 80, 1-29.

Vincent, C. H. (1970). The generation of truly random binary numbers. Journal of Physics E, 3, 594-598.

von Lucadou, W. (1987a). A multivariate PK experiment: Part I. An approach combining physical and psychological conditions of the PK process. European Journal of Parapsychology, 6, 305-345.

von Lucadou, W. (1987b). A multivariate PK experiment: Part III. Is PK a real force? The results and their interpretation. European Journal of Parapsychology, 6, $369-428$.

von Lucadou, W. (1988a). The model of pragmatic information (MPI). In D. H. Weiner \& R. L. Morris (Eds.) Research in parapsychology 1987 (pp. 18-22). Scarecrow Press.

von Lucadou, W. (1988b). A multivariate PK experiment with unidirectional correlated RNGs. In D. H. Weiner \& R. L. Morris (Eds.) Research in parapsychology 1987 (pp. 22-26). Scarecrow Press.

von Lucadou, W. (1994). Locating psi bursts: Correlations between psychological characteristics of observers and observed quantum physical fluctuations. In E. W. Cook \& D. L. Delanoy (Eds.) Research in parapsychology 1991 (pp. 39-43). Scarecrow Press.

von Lucadou, W. (1995). The model of pragmatic information (MPI). European Journal of Parapsychology, 11, 58-75.

von Lucadou, W. (2006). Self-organization of temporal structures-A possible solution for the intervention problem. In D. P. Sheehan (Ed.) Frontiers of time, retrocausation-Experiment and theory (pp. 293-315). AIP Conference Proceedings Vol. 863. American Institute of Physics.

von Lucadou, W., Lay, B., \& Kunzmann, H. (1987). A multivariate PK experiment: Part II. Relationships between psychological variables. European Journal of Parapsychology, 6, 347-368.

Walach, H. (2014). Mind-matter interactions-On the rollercoaster from data to theory and back again. Aquém e além do cérebro: 100 simpósio da Fundação Bial [Behind and beyond the brain: Proceedings of the roth Bial Foundation symposium] (pp. 85-114). Fundação Bial.

Walach, H., Horan, M., Hinterberger, T., \& von Lucadou, W. (2020). Evidence for anomalistic correlations between human behavior and a random event 
generator: Result of an independent replication of a micro-PK experiment. Psychology of Consciousness: Theory, Research, and Practice, 7, 173-188. https:// dx.doi.org/10.1037/cnsoooo199

Walker, E. H. (1975). Foundations of paraphysical and parapsychological phenomena. In L. Oteri (Ed.) Proceedings of an international conference: Quantum physics and parapsychology (pp. 1-44). Parapsychology Foundation. Walker, E. H. (1984). A review of criticisms of the quantum mechanical theory of psi phenomena. Journal of Parapsychology, 48, 277-332.

Walker, E. H. (2000). The physics of consciousness: Quantum minds and the meaning of life. Perseus Publishing.

Watkins, M. R., \& Walker, J. (1996). The RetroPsychoKinesis Project (RPKP). https:// www.fourmilab.nl/rpkp

Watt, C. A., \& Kennedy, J. E. (2015). Lessons from the first two years of operating a study registry. Frontiers in Psychology, 6, 173. https://dx.doi.org/10.3389/ fpsyg.2015.00173

Watt, C. A., \& Kennedy, J. E. (2017). Options for prospective meta-analysis and introduction of registration-based prospective meta-analysis. Frontiers in Psychology, 7, 2030. https://dx.doi.org/10.3389/fpsyg.2016.02030

Weaver, Z. (2015). Other realities? The enigma of Franek Kluski's mediumship. White Crow Books.

Weiner, D. H. (1978). High- vs. low-risk condition in a computerized PK game [Abstract]. Journal of Parapsychology, 42, 64-65.

Weiner, D. H. (1979). A test of the relationship between PK and motor skill [Abstract]. Journal of Parapsychology, 43, 40-41.

White, R. A. (1976). The limits of experimenter influence on psi test results: Can any be set? Journal of the American Society for Psychical Research, 70, 333-369.

White, R. A. (1977). The influence of the experimenter motivation, attitudes and methods of handling subjects in psi test results. In B. B. Wolman (Ed.) Handbook of parapsychology (pp. 273-301). Van Nostrand Reinhold.

Williams, B. J. (2015). Psychic phenomena and the brain: Exploring the neuropsychology of psi. AIPR Monograph No. 3. Australian Institute of Parapsychological Research

Wilson, D. B., \& Shadish, W. R. (2006). On blowing trumpets to the tulips: To prove or not to prove the null hypothesis-Comment on Bösch, Steinkamp, and Boller (2006). Psychological Bulletin, 132, 524-528.

Wright, S. H. (1998). Experiences of spontaneous psychokinesis after bereavement. Journal of the Society for Psychical Research, 62, 385-395.

Young, H. D. (2012). Sears and Zemansky's college physics (9th ed.). Addison-Wesley/ Pearson.

Zenchenko, T. A., \& Breus, T. K. (2021). The possible effect of space weather factors on various physiological systems of the human organism. Atmosphere, 12, 346. https://dx.doi.org/10.3390/atmos12030346 


\begin{tabular}{|c|c|c|c|c|}
\hline \multicolumn{5}{|c|}{$\begin{array}{l}\text { APPENDIX TABLE } \\
\text { Retroactive Psychokinesis Experiments Using Pre-Recorded RNG Targets }\end{array}$} \\
\hline Study & Description & Pub Vol \# & $z$-score & $p$-value \\
\hline 1 & Bierman \& Houtkooper (1975) - High-Speed RNG & $\operatorname{EJP} 1(1)$ & 0.89 & .186 \\
\hline 2 & H. Schmidt (1976) - Pre-Recorded Targets I & JASPR 70 & 3.14 & .0008 \\
\hline 3 & H. Schmidt (1976) - Pre-Recorded Targets 2 & JASPR 70 & 4.22 & .00001 \\
\hline 4 & H. Schmidt (1976) - Pre-Recorded Targets 3-Pilot* & JASPR 70 & 2.41 & .008 \\
\hline 5 & $\begin{array}{l}\text { H. Schmidt (1976) - Pre-Recorded Targets } \\
\text { 3-Confirmatory* }\end{array}$ & JASPR 70 & 2.03 & .021 \\
\hline 6 & $\begin{array}{c}\text { Millar \& Mackenzie (1977) - Intentional \& } \\
\text { Unintentional PK }\end{array}$ & RIP 1976 & 0.00 & .50 \\
\hline 7 & Houtkooper (1977) - Direct \& Random Retro-PK-Pilot & $\operatorname{EJP}_{1}(4)$ & $2.28^{\star \star}$ & .011 \\
\hline 8 & $\begin{array}{l}\text { Houtkooper (1977) - Direct \& Random Retro-PK- } \\
\text { Confirmation }\end{array}$ & $\operatorname{EJP} 1(4)$ & $-0.55^{\star *}$ & .709 \\
\hline 9 & Broughton et al. (1978) - PK Experimenter Psi Effect & RIP 1977 & 0.00 & .50 \\
\hline 10 & Davis \& Morrison (1978) - PK Multiple Feedback Test* & RIP 1977 & 0.00 & .50 \\
\hline 11 & $\begin{array}{l}\text { H. Schmidt (1978) - Take-Home PK Test Pre- } \\
\text { Recorded Targets }{ }^{*}\end{array}$ & RIP 1977 & 3.34 & .0004 \\
\hline 12 & $\begin{array}{l}\text { H. Schmidt (1978) - Take-Home PK Test Pre- } \\
\text { Recorded Targets } 2^{\star}\end{array}$ & RIP 1977 & -1.13 & .871 \\
\hline 13 & Terry \& Schmidt (1978) - Conscious \& Subconscious PK & RIP 1977 & -3.07 & .998 \\
\hline 14 & H. Schmidt (1979) - PK Test Stroboscopic Light* & RIP 1978 & 2.90 & .002 \\
\hline 15 & $\begin{array}{l}\text { Morrison \& Davis (1979) - PK Immediate, Delayed, } \\
\text { Multiple Feedback-Delay }{ }^{*}\end{array}$ & RIP 1978 & 0.20 & .421 \\
\hline 16 & $\begin{array}{l}\text { Morrison \& Davis (1979) - PK Immediate, Delayed, } \\
\text { Multiple Feedback-Delay } 4^{*}\end{array}$ & RIP 1978 & 0.19 & .425 \\
\hline 17 & $\begin{array}{l}\text { Houtkooper et al. (1980) - OT Identity \& Feedback- } \\
\text { Pilot Expt. }\end{array}$ & $\mathrm{EJP}_{3}(3)$ & 3.23 & .0006 \\
\hline 18 & $\begin{array}{l}\text { Houtkooper et al. (1980) - OT Identity \& Feedback- } \\
\text { 2nd Expt. Observe }\end{array}$ & EJP $3(3)$ & 0.37 & .356 \\
\hline 19 & $\begin{array}{l}\text { Houtkooper et al. (1980) - OT Identity \& Feedback- } \\
\text { 2nd Expt. Hidden }\end{array}$ & $\mathrm{EJP}_{3}(3)$ & -2.45 & .993 \\
\hline 20 & $\begin{array}{l}\text { Braud (1981)-Long Distance Time-Displaced PK } \\
\text { Selected Participants* }\end{array}$ & RIP 1980 & 0.00 & .50 \\
\hline 21 & $\begin{array}{l}\text { H. Schmidt (1981) - PK Pre-Recorded \& Pre- } \\
\text { Inspected Seed-Pilot* }\end{array}$ & $J P_{45}$ & 2.12 & .017 \\
\hline 22 & $\begin{array}{l}\text { H. Schmidt (1981)-PK Pre-Recorded \& Pre- } \\
\text { Inspected Seed-Unselected* }\end{array}$ & $J_{4} P_{5}$ & 2.19 & .014 \\
\hline 23 & $\begin{array}{l}\text { H. Schmidt (1981)-PK Pre-Recorded \& Pre- } \\
\text { Inspected Seed-Selected* }\end{array}$ & JP 45 & 3.42 & .0003 \\
\hline
\end{tabular}




\begin{tabular}{|c|c|c|c|c|}
\hline 24 & $\begin{array}{c}\text { Talbert \& Debes (1982)-Time-Displaced PK Effects } \\
\text { Varying Feedback* }\end{array}$ & RIP 1981 & 0.00 & .50 \\
\hline 25 & $\begin{array}{l}\text { H. Schmidt (1985) - Addition Effect PK Pre- } \\
\text { Recorded Targets }\end{array}$ & $J P_{49}$ & 2.59 & .005 \\
\hline 26 & Bierman (1985) - PK Test for Babies-Amsterdam & $\mathrm{EJP}_{5}$ & $-2.08^{\star \star}$ & .981 \\
\hline 27 & Bierman (1985) - PK Test for Babies-Bristol & EJP 5 & $1.00^{* *}$ & .159 \\
\hline 28 & $\begin{array}{l}\text { Schmidt et al. (1986) - Channeling PK Independent } \\
\text { Observers }\end{array}$ & $J P_{50}$ & 2.71 & .003 \\
\hline 29 & $\begin{array}{l}\text { Schmidt \& Schlitz (1989) - Large-Scale Pilot PK Pre- } \\
\text { Recorded Events }\end{array}$ & RIP 1988 & 1.66 & .048 \\
\hline 30 & $\begin{array}{c}\text { Schmidt \& Braud (1993) - New PK Tests } \\
\text { Independent Observer }\end{array}$ & $J P 57$ & 1.98 & .024 \\
\hline 31 & $\begin{array}{l}\text { Schmidt et al. (1994) - Channeling PK Independent } \\
\text { Observers } 2\end{array}$ & RIP 1991 & 0.62 & .268 \\
\hline 32 & $\begin{array}{l}\text { Michels (1993) - PK Tests With and Without } \\
\text { Skeptics }\end{array}$ & Skepter (4) & 1.64 & .05 \\
\hline 33 & Schmidt \& Stapp (1993) - Pre-Observation PK Effects & $J P 57$ & 1.23 & .109 \\
\hline 34 & $\begin{array}{l}\text { H. Schmidt (1997) - RNGs \& Living Systems } \\
\text { Targets-RNG }\end{array}$ & JASPR 91 & 1.68 & .046 \\
\hline 35 & $\begin{array}{l}\text { Schmidt \& Dalton (1999) - Repeated Efforts Pre- } \\
\text { Recorded 1-Multi-PK }\end{array}$ & $J P 63$ & 0.06 & .476 \\
\hline 36 & $\begin{array}{l}\text { Schmidt \& Dalton (1999) - Repeated Efforts Pre- } \\
\text { Recorded 2-Meditation }\end{array}$ & $J P 63$ & 0.66 & .255 \\
\hline 37 & $\begin{array}{l}\text { Schmidt \& Dalton (1999) - Repeated Efforts Pre- } \\
\text { Recorded 3-Click-PK }\end{array}$ & $J P 63$ & -1.99 & .977 \\
\hline 38 & H. Schmidt (2000) - PK Tests Pre-Sleep State - Pilot & $J P 64$ & 2.45 & .007 \\
\hline 39 & $\begin{array}{l}\text { H. Schmidt (2000) - PK Tests Pre-Sleep State - } \\
\text { Confirmatory }\end{array}$ & $J P 64$ & 2.24 & .013 \\
\hline 40 & $\begin{array}{l}\text { H. Schmidt (2000)-PK Tests Pre-Sleep State-Added } \\
\text { Day Test }\end{array}$ & $J P 64$ & 1.01 & .156 \\
\hline 41 & Dobyns (2006)-PEAR Retrocausal REG & AIP Proc 863 & 1.28 & .100 \\
\hline 42 & $\begin{array}{l}\text { Watkins \& Walker (1996-9/2021) - } \\
\text { RetroPsychoKinesis Project Summary }\end{array}$ & Fourmilab.nl & -0.27 & .394 \\
\hline All & & & 6.82 & $10^{-12}$ \\
\hline \multicolumn{5}{|c|}{$\begin{array}{l}\text { Note: Following the approach taken by Bierman (1998), experiments that obtained null } \\
\text { results and did not provide any other details or descriptive statistics in their reports with } \\
\text { which to calculate a z-score estimate were arbitrarily assigned a z-score of zero. } \\
\text { AIP Proc-American Institute of Physics Proceedings; EJP-European Journal of } \\
\text { Parapsychology; JASPR-Journal of the American Society for Psychical Research; JP-Journal of } \\
\text { Parapsychology; RIP-Research in Parapsychology } \\
\text { * Studies missing from initial Bierman (1998) analysis } \\
\text { ** Scores corrected from Bierman (1998) analysis; original sources consulted }\end{array}$} \\
\hline
\end{tabular}

